\title{
Perception and action in nicotine addiction : fMRI and behavioral studies of sensorimotor and multisensory processes in smokers and non-smokers
}

Citation for published version (APA):

Yalachkov, Y. V. (2011). Perception and action in nicotine addiction : fMRI and behavioral studies of sensorimotor and multisensory processes in smokers and non-smokers. [Doctoral Thesis, Maastricht University]. Datawyse / Universitaire Pers Maastricht. https://doi.org/10.26481/dis.20110317yy

Document status and date:

Published: 01/01/2011

DOI:

10.26481/dis.20110317yy

Document Version:

Publisher's PDF, also known as Version of record

Please check the document version of this publication:

- A submitted manuscript is the version of the article upon submission and before peer-review. There can be important differences between the submitted version and the official published version of record.

People interested in the research are advised to contact the author for the final version of the publication, or visit the DOI to the publisher's website.

- The final author version and the galley proof are versions of the publication after peer review.

- The final published version features the final layout of the paper including the volume, issue and page numbers.

Link to publication

\footnotetext{
General rights rights.

- You may freely distribute the URL identifying the publication in the public portal. please follow below link for the End User Agreement:

www.umlib.nl/taverne-license

Take down policy

If you believe that this document breaches copyright please contact us at:

repository@maastrichtuniversity.nl

providing details and we will investigate your claim.
}

Copyright and moral rights for the publications made accessible in the public portal are retained by the authors and/or other copyright owners and it is a condition of accessing publications that users recognise and abide by the legal requirements associated with these

- Users may download and print one copy of any publication from the public portal for the purpose of private study or research.

- You may not further distribute the material or use it for any profit-making activity or commercial gain

If the publication is distributed under the terms of Article $25 \mathrm{fa}$ of the Dutch Copyright Act, indicated by the "Taverne" license above, 


\title{
Perception and action in nicotine addiction
}

\author{
fMRI and behavioral studies of \\ sensorimotor and multisensory processes \\ in smokers and non-smokers
}

Yavor Vasilev Yalachkov 


\section{Colophon}

(C) Copyright Y Yalachkov, Frankfurt 2011

Datawyse bv, Universitaire Pers Maastricht ISBN 9789461590428 


\title{
Perception and action in nicotine addiction
}

\author{
fMRI and behavioral studies of \\ sensorimotor and multisensory processes \\ in smokers and non-smokers
}

\author{
Dissertation
}

To obtain the degree of Doctor at Maastricht University, on the authority of the Rector Magnificus, Prof. dr. G.P.M.F. Mols in accordance with the decision of the Board of Deans, to be defended in public on Thursday, March 17 $7^{\text {th }}$ 2011, at 16.00 hours.

by

\section{Yavor Vasilev Yalachkov}

Born October $8^{\text {th }} 1985$ in Plovdiv

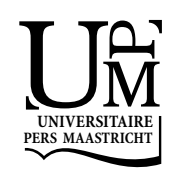




\section{Supervisor}

Prof. dr. Rainer Goebel

\section{Co-supervisors}

Prof. dr. Jochen Kaiser

Dr. Marcus J. Naumer

\section{Assessment Committee}

Prof. dr. Bernadette Jansma (voorzitter)

Prof. dr. Leo Blomert

Dr. Amanda Kaas

Prof. dr. Andrea Kübler (University of Würzburg)

Dr. Matt Field (University of Liverpool)

The research reported in the present dissertation was conducted at the Institute of Medical Psychology, Goethe-University, Frankfurt am Main, Germany. 


\section{Contents}

Chapter 1 General introduction to the neuroscience of addiction 7

Chapter 2 Sensory and motor aspects of addiction

Chapter 3 Brain regions related to tool use and action knowledge reflect nicotine addiction

Chapter 4 Sensory modality of smoking cues modulates neural cue reactivity

Chapter 5 Smoking experience modulates the cortical integration of vision and haptics

Chapter 6 General discussion 

Chapter 1

General introduction to

the neuroscience of addiction 


\section{A brief history of drug taking behavior}

The present $\mathrm{PhD}$ thesis studies the sensorimotor and multisensory aspects of nicotine addiction, which have been rather neglected by addiction research. A brief overview of well-established findings from the field of addiction science might facilitate the reader's understanding of addiction in general. It should also help to motivate the aim of the present thesis: to complement the neuroscience of addiction by elucidating its sensorimotor and multisensory aspects.

Drug taking behavior has been a relevant social phenomenon for a long time. Traces of drug use have been found in tissue samples from Egyptian mummies, dating back to 1100 years B.C., but drug use (perhaps just as abuse and dependence) seems to be much older than that, possibly as old as mankind. 30000 years B.C. some hallucinogens were used, perhaps in the context of certain rituals or shaman cults. On the other hand, the negative consequences of prolonged drug use were clearly demonstrated, accepted and studied only much later. 500 years A.D. the abuse of hashish in Egypt was prohibited. The addictive potential of morphine and other opiates became strikingly clear after the German-French war of 1870/1871, where morphine was widely used to alleviate pain (Schmidbauer and VomScheidt, 2004).

Nowadays substance use and addiction cause a significant burden to individuals and societies throughout the world. According to The World Health Organization, there are currently 2 billion alcohol users, 1.3 billion smokers and 185 million drug users worldwide. Furthermore, tobacco, alcohol and illegal drugs have contributed together $12.4 \%$ of all deaths in the year 2000 . It has been estimated that these substances account for $8.9 \%$ of total years of life lost. Among these substances, tobacco alone has been responsible for the highest percentage of mortality ( $8.8 \%$ or 4.9 million) and total years of life lost (4.1\% or 59.1 million). Unless current trends are not stopped, it is expected that the tobacco-driven number of deaths will rise to 10 million per year by the 2020 s or early $2030 \mathrm{~s}$ (http://www.who.int/substance_abuse/facts/global_burden/en/index.html, online access on 29.03.2010).

This burden seems even harder to cope with, when the high relapse rates are taken into account. For example, in nicotine addiction even the most effective therapeutic approaches have long-term success rates of only 30\% (Piasecki, 2006). Although the physiological dependence and the withdrawal symptoms of addiction may vanish when remaining abstinent for a certain period of time, it is the psychological dependence that often causes addicts to return to drug use, although they may explicitly express their motivation to stay "clean". This indicates that scientific research on addiction should be intensified and its empirical findings should be integrated in both medical care and psychotherapy standards in order to achieve better treatment outcomes.

\section{Dependence and addiction}

The various forms of substance dependence are outlined in the Diagnostic and Statistical Manual of Mental Disorders (APA, 2000) and the International Classification of Diseases (WHO, 1996). Importantly, one can recognize that there 
are several behavioral, cognitive, and physiological symptoms, which are described in both DSM-IV and ICD-10 as most typical for the dependence diagnosis: a strong desire, need or compulsive drive to take the substance, difficulties to control the process of drug taking and persisting drug use in spite of harmful consequences. Furthermore, over time drug taking and drug seeking behavior dominate over other activities and duties. The abuse of certain drugs may lead to tolerance (one needs a higher quantity of the drug to achieve the initially experienced effect) and physiological withdrawal syndrome (very unpleasant symptoms that vary across the different drugs of abuse like sweating, tremor, nausea, tachycardia, hypertension, dysphoria, etc. when the regular drug dose has not been taken), which represent further characteristics of dependence. In the scientific and clinical community the physiological dependence (in terms of tolerance and/or withdrawal symptoms) is usually distinguished from the psychological dependence. The term "addiction" is used mostly for the psychological aspects of the chronic disease "substance dependence" and stresses the compulsive behavioral and cognitive patterns related to drug seeking and drug taking.

The psychological dependence or addiction usually requires a long time of abstinence, qualified psychotherapy, and sometimes also social reintegration. Addiction is also responsible for relapses, which can occur even after prolonged abstinence and intensive medical and psychological treatment. The present thesis focuses on these hardly controllable aspects of drug seeking and drug taking behavior.

\section{Models of addiction}

\section{Some initial considerations}

Addiction can be best explained by a biopsychosocial model, which integrates diverse factors: biological (e.g. genetic, molecular, cellular, physiological, etc), psychological (e.g. behavioral, cognitive, emotional, motivational, stress-related, etc.), and social (e.g. peers, family, school, etc.). However, the present thesis focuses on the neuroscientific aspects of nicotine addiction and aims at revealing its sensorimotor and multisensory aspects by non-invasive neuroimaging and experimental behavioral methods. Therefore, the following overview will concentrate on models, which cover the role of the brain in establishing and maintaining drug seeking and drug taking behavior.

\section{The pathological motivation of addiction and its neural basis}

Drugs of abuse are able to produce very strong euphoric feelings in the subjects taking them. Some scientists have therefore argued that the desire to experience the positive hedonic effects of the drug motivates the addict to take the drug (McAuliffe and Gordon, 1974), and leads to addictive behaviors. This hedonic view is very close to (or even identical with) the common-sense view of addiction: people take drugs because they like them. The motivation to take drugs is established due to an assumed causal relationship between liking and taking drugs. Typical findings which have been interpreted as evidence for a link between the appetitive, positive 
reinforcing effects of drugs and addictive behaviors stem from paradigms in which animals would self-administer drugs at high rates, sometimes leading to dependence or intoxication (Stewart et al., 1984).

The next step in developing a positive reinforcement model of addiction was to search for the neural mechanisms of hedonic pleasure, which may play a critical role in addiction. Dopamine released in the ventral striatum/nucleus accumbens (NAcc) by projections from the ventral tegmental area (VTA) has been suggested as a "pleasure neurotransmitter" (Wise, 1980). Natural rewards such as food or sex, but also drugs like psychostimulants, are known to increase the extracellular dopamine concentration in the NAcc (Wise, 2004). This effect can be observed when the participants receive a small or moderate drug dose or when addicts are exposed to stimuli that are linked to the drugs of abuse through classical conditioning. Therefore, some researchers have hypothesized that enhanced dopamine concentration in the NAcc during an acute intoxication or an exposure to conditioned stimuli would be correlated with the (expectation of) hedonic feelings. Indeed, some studies found a correlation between the reported "high" feeling and the dopamine transporter occupancy (Volkow et al., 1999).

However, there are numerous studies which contradict the main predictions of the hedonic view. Although the hedonic view can explain very well the initial stages of drug abuse and addiction, it cannot provide robust evidence why addicts compulsively continue to take drugs during the next stages of their addiction, when they face problems such as health risks and delinquency (to name just a few of them) and despite their wish to end the drug consumption. In a more advanced stage of the disease, addicts no longer have the strong association between the hedonic effects of the drug and the motivation for taking it. In fact, many of them state that they do not feel any hedonic pleasure when taking their dose but nevertheless feel themselves simply driven to continue taking it. Drug taking may increase dramatically over time as addiction develops, but the pleasure induced by a given dose of a drug is not found to increase (Robinson and Berridge, 1993). Such observations indicate that the relationship between the subjective pleasurable effects of drugs and drug taking is not causal, leading to the conclusion that the hedonic view is unable to explain the whole spectrum of addictive behaviors. Similar evidence comes from experiments using objective measurements of hedonic reactions in human infants and nonhuman animals, i.e., affective gestures and mimics elicited by hedonic impact (Berridge, 2000). Moreover, in animal experiments dopamine manipulations such as lesions of dopamine projections, administration of neuroleptic drugs, genetic manipulations, or amphetamine microinjections into the NAcc do not change the "liking" (i.e. hedonic) reactions. Similarly, patients with the dopamine deterioration of Parkinson's disease show normal subjective pleasure ratings for sweet food rewards [see (Berridge, 2007) for an excellent review of the relevant dopamine literature]. More importantly, the correlation between dopamine levels in the NAcc and hedonic mood ratings was found to be lower than the one between dopamine levels in the NAcc and ratings of "want"-feelings for the same reward (Leyton et al., 2002). Furthermore, recent studies using invasive electrophysiology in animals have also ascribed a very different role of dopamine in the context of reward and addiction. Phasic bursts of dopaminergic VTA cells have been shown to reflect the reward prediction error on the neuronal level. For example, midbrain dopaminergic cells change their activity from tonic to phasic, when the individual receives a reward, 
which is higher as he or she has expected (positive reward prediction error). Accordingly, an inhibition of the cells can be observed, when the reward is smaller than expected (negative reward prediction error), and no change in the activity of the dopaminergic system occurs, when the amount of the reward equals the expectation (reward prediction error equals zero) (Schultz et al., 1997; Schultz and Dickinson, 2000; Schultz, 2007b). Once the individual has learnt the incentive value of a reward, its midbrain dopaminergic system would not respond anymore with phasic bursts to the reward if the received reward amount stays the same, i.e., when there is no reward prediction error. However, due to their direct pharmacological effects (e.g. blocking dopamine transporters or increasing presynaptic intracellular dopamine concentrations, etc.), most drugs of abuse differ from natural rewards and can elevate the dopamine levels in the NAcc and the cortex even after repeated drug consumption by the addicted individual. Thus, although the drug effects (and thus the expected reward) are well-known to the brain reward system, each single drug consumption results in a positive reward prediction error and is rated by the brain reward system as "better than expected", leading to pathologically high incentive values ascribed to the drug (Montague et al., 2004; Redish, 2004).

If the reward is repeatedly paired with environmental stimuli, the phasic activation of the dopaminergic cells shifts from the reward itself to the cues predicting it (Schultz et al., 1997; Schultz and Dickinson, 2000; Tobler et al., 2005; Schultz, 2007b, a). Thus, exposure to reward-related cues can lead to enhanced dopamine release, which reveals that dopamine can encode also the expected reward associated with the presented cue, i.e. the incentive value of a particular environmental stimulus. The incentive-sensitization theory integrates these findings in a model explaining the pathologically high motivation for drug taking in addiction (Robinson and Berridge, 1993, 2001; Berridge, 2007). It assumes that addictive drugs are able to induce long-lasting changes in those brain structures that are related to processes of incentive motivation and reward and that these brain systems become sensitized to drugs and drug-associated stimuli. Thus, the sensitized systems which do not mediate the "liking" subcomponent of reward, i.e. the euphoric or hedonic effects of drugs, but instead encode another subcomponent of reward, the so-called "incentive salience" and "wanting"-processes, are responsible for the compulsive drug taking behavior. The process of attributing a motivational salience ("incentive salience") is crucial for transforming the psychological and neural representations of the stimuli, so that they attract attention, become wanted, and most importantly elicit compulsive drug seeking and drug taking behaviors. Interestingly, this can occur not only without being aware of "wanting" something (Winkielman et al., 2005), but also in the absence of subjective pleasure (Lamb et al., 1991). The high incentive values of drugs and drug-related stimuli gained through the sensitization process result in a powerful drive, motivating addicts to seek and take the drug. This neural sensitization is suggested to persistently take place in reward-related brain systems (such as the NAcc and the VTA) leaving subjects vulnerable to relapse even after a long time without taking drugs. Indeed, recent research has demonstrated that drugs of abuse hijack the cellular and molecular mechanisms of learning and memory and induce significant neuroplastic processes in the NAcc and VTA (Hyman and Malenka, 2001; Hyman, 2005; Hyman et al., 2006; Kauer and Malenka, 2007; Russo et al., 2010). Also manipulations of the dopaminergic brain reward system have been found to modulate the degree of 
"wanting" but not "liking" rewards (Cagniard et al., 2006). Further evidence for the relevance of the incentive-sensitization theory comes from human neuroimaging studies, which show that the neural response in the NAcc to intranasal cocaine selfadministration is predicted by the lifetime history of stimulant drug use and that in healthy participants who have been given just three doses of amphetamine there is a greater psychomotor response and increased dopamine release in the NAcc relative to the initial dose even one year after the third dose (Boileau et al., 2006; Cox et al., 2009).

A brain region which has been consistently reported to be activated during craving for drugs is the orbitofrontal cortex (OFC). Interestingly, OFC activation has been demonstrated also in numerous experiments studying behavioral drive and the value of future events. Since it is connected to the NAcc, its participation in motivation is not surprising. It has been suggested to be particularly involved in representing the value of a stimulus and the outcome of an action as well as integrating them in the course of behavior regulation (Schoenbaum et al., 2006; Schoenbaum and Shaham, 2007). Especially when values change or are even reversed, the OFC plays an important role (Cools et al., 2002; Morris and Dolan, 2004). In experiments using the Iowa gambling task to study decision making, humans with OFC lesions are unable to learn from negative consequences as fast and accurately as healthy subjects (Bechara et al., 1999). Thus, the patients fail to integrate information about occasional large penalties into their future choices. The relevance of these findings for addiction has been demonstrated by a growing number of studies reporting that OFC activation in addicted subjects is stronger than in healthy subjects when they report craving due to exposure to drug-related stimuli or to a given drug dose (Volkow et al., 2002). Furthermore, prolonged drug taking, especially of psychostimulants, leads not only to considerable functional changes in the OFC (Dom et al., 2005), but also results in structural alterations, such as a reduction in dendritic spine density (Crombag et al., 2005). Essentially, reduced metabolism in the OFC and decreased availability of striatal D2 dopamine receptors in drug abusers in comparison to control subjects have been observed and shown to be intercorrelated, suggesting a crucial disruption of important parts of the brain motivational system (Volkow et al., 2009). The baseline hypoactivity in OFC has been interpreted as an impaired control over compulsive drug-related behavior as well as a reduced capacity of the prefrontal cortex to initiate behaviors in response to biological rewards, leading to a decreased motivation for non-drug activities (Volkow et al., 2002). On the other hand, OFC hyperactivation in response to drug stimuli represents the intense motivational drive and craving experienced by addicts upon confrontation with drug cues (Kalivas and Volkow, 2005).

\section{Drug taking habits and automatized action schemata}

Recent research has hypothesized that the neural mechanisms which are responsible for the development and expression of stimulus-response habits may facilitate the formation of drug taking habits in addicts. The dorsal striatum consisting of the putamen and the dorsal nucleus caudatus is known to be responsible for the acquisition of behavioral habits in terms of strong "stimulus-response" associations (Packard and Knowlton, 2002). On the other hand, there is growing evidence that drugs of abuse can influence long-lasting processes of synaptic plasticity in the 
striatum such as long-term potentiation and long-term depression that are important for the development of habits (Gerdeman et al., 2003). It has been assumed that the progress of addiction from its initial to its final stages is accompanied by a corresponding change from a strongly goal-oriented (mediated by the NAcc) to a more action-oriented behavioral pattern. This compulsive behavior has been associated with habitual stimulus-response learning and the involvement of the dorsolateral striatum (Everitt and Robbins, 2005). For example, it has been shown that neuroadaptation markers are found mainly in the ventral striatum (NAcc) during the initial stages of addictive behavior, while prolonged experience of drug seeking leads to a shift of these prominent neuroplastic changes towards the dorsal striatum (Porrino et al., 2004). Most importantly, a recent study has provided evidence that the expression of drug seeking habits, which are under the control of associated cues, depends upon a serial connectivity linking the ventral with the dorsal striatum (Belin and Everitt, 2008). This points out that addiction is indeed a disease which progressively engages not only the reward but also the habit system. Connections between the two systems allow the brain to establish a powerful link between a particular stimulus and a subsequent, rewarded reaction. In this context, the progressive involvement of the dorsal striatum accounts for the growing strength of the stimulus-reaction association, which becomes automatized and insensitive to reinforcer devaluation.

An interesting complementary approach to automatized and habitual mechanisms of addiction is the cognitive model proposed by Tiffany (Tiffany, 1990; Tiffany and Carter, 1998; Tiffany and Conklin, 2000). The model assumes that drug use behavior is represented in the form of automatized action schemata. The repeated practice of the same cognitive or motor task leads to the development of skilled behavior which requires less effort, and is rapidly and accurately performed almost without any awareness of the component actions. This transformation due to extended practice has also been conceptualized as the development of automaticity (Shiffrin and Schneider, 1977). Thus, drug use behavior can be conceptualized as an overlearned activity, similar to habitual activities like tooth brushing, driving a car, etc.. In other words, Tiffany describes drug use behavior as a constellation of specific sensorimotor skills involving drug acquisition and drug consumption (Tiffany, 1990). These skills have been developed to such a degree that they have gained the character of automatic processes. Thus, they become stimulus-bound (i.e. they are readily activated by conditioned stimuli) and can be triggered and performed without much effort and awareness. The form in which they are assumed to be stored and represented is called automatized action schemata or action plans, which contain the information for initiating and successfully carrying out the behavioral sequences.

\section{Impaired executive functions contribute to drug seeking and drug taking}

Impairment of executive functions in terms of decreased cognitive and motivational control is another central aspect of the addiction research. Behavioral data have demonstrated that addicts have difficulties in GO-NOGO tasks and strong deficits in inhibitory control processes and decision making. Hester \& Garavan (Hester and Garavan, 2004) showed that the compromised control abilities of cocaine users are associated with reduced activities in anterior cingulate and right prefrontal cortices 
and that the addicted subjects showed an over-reliance on the left cerebellum, a compensatory pattern previously also seen in alcohol addicts. A possible mechanism through which prefrontal and anterior cingulate activity might be diminished is the repeated exposure to a hyperdopaminergic state. The prefrontal and anterior cingulate cortices are thought to be crucial for exerting cognitive control of behavior, optimizing decision-making, and modulating working memory and attention processes. In human studies the dorsolateral prefrontal cortex (DLPFC) has been reported to be reactivated when automatized behavior had to be cognitively controlled (Kübler et al., 2006). The evidence for an essential role of this brain structure in decision-making has been supported and extended by studies, in which transcranial direct current stimulation applied to the DLPFC reduced the appetite for risk during ambiguous decision making (Fecteau et al., 2007). Recent neurobiological research inspired by the described neuroimaging studies has elucidated the cellular underpinnings of the impaired ability of the prefrontal cortex to control drug seeking behavior. Interestingly, while glutamate homeostasis plays an essential role for maintaining the ability of the prefrontal cortex to exert control over motivational drives from limbic brain regions, addiction seems to be related to an imbalance between synaptic and non-synaptic glutamate. The disrupted glutamate homeostasis in addiction has been suggested to result in impaired communication between the prefrontal cortex and nucleus accumbens (Kalivas et al., 2005; Kalivas and Volkow, 2005; Kalivas, 2009).

\section{Preliminary conclusion}

Different studies of addiction have focused on diverse neurobiological explanation models. While none of them is able to fully explain such a complex phenomenon as addiction, each of them can at least contribute to a better understanding of some of its particular aspects. Models aiming at integrating these various points of view and stressing their individual roles in different stages of addiction, appear to be more appropriate (Baler and Volkow, 2006; Goldstein et al., 2009; Koob and Volkow, 2010). While the hedonic view and positive reinforcement aspects might be especially important during the initial stages of drug abuse, incentive-sensitization and the establishment of habits may be crucial both for the pathologically high incentive values of drugs and drug-cues and for the compulsive behavioral pattern of drug taking. The reduced ability to integrate negative experiences into behavioral control as well as compromised decision-making and diminished behavioral inhibition may be responsible for the uncontrollable and repeated expression of this behavioral pattern. And finally, all these factors may contribute to the danger that addicts relapse even after a prolonged time of abstinence.

\section{Neural correlates of nicotine addiction}

Studies of nicotine addiction have provided strong evidence that most of its neural correlates are similar to the ones demonstrated for other substances. More precisely, the involvement of brain regions such as VTA, striatum and prefrontal cortex in developing drug seeking behavior, cue reactivity, and relapse in the context of nicotine addiction has been clearly demonstrated by numerous studies [see (Chiamulera, 2005; Brody, 2006) for special reviews on the neuroimaging of 
nicotine addiction]. Interestingly, the insula has recently gained particular attention as one of the key brain regions contributing to relapse in nicotine addiction. Damage to the insula in humans is strongly linked to an abrupt and robust decrease in smoking and craving (Naqvi et al., 2007). One possible explanation for the involvement of the insula in craving and addiction is its central role for integrating interoceptive states into conscious feelings and into decision-making processes (Naqvi and Bechara, 2009). This is particularly well described by the words of a patient who has experienced damage to his insula and quit his daily intensive smoking immediately after that: he stated that he quit because his "body forgot the urge to smoke" (Naqvi et al., 2007).

\section{Novel aspects of addiction and sensorimotor/multisensory processes}

There is mounting evidence demonstrating that sensorimotor processes, which have not received much attention in previous addiction research, may be relevant for the understanding of nicotine addiction and its neural underpinnings. Brody et al. (Brody et al., 2002) have found a positive correlation between craving after exposure to cigarette cues and activation of the right sensorimotor cortex in heavy smokers. The severity of nicotine dependence correlated with the activation of motor preparation and imagery networks such as primary and premotor cortex and supplementary motor area (Smolka et al., 2006). Interestingly, smokers trying to resist craving during cigarette cue exposure showed BOLD signal reductions in primary sensory and motor cortices (Brody et al., 2007). Crucially, fMRI activation of sensory and motor cortices in cocaine addicts after exposure to cocaine-related cues can predict relapse (Kosten et al., 2006).

There is also evidence that the sensory modality in which the drug cue is presented can modulate the psychophysiological cue reactivity (Johnson et al., 1998). The influence of the used sensory modality on the measures of neural and behavioral cue reactivity is more than essential, since a particularly important aim of the cue reactivity research is to establish a reliable biomarker of drug addiction and study the factors which are able to affect it.

These findings suggest that both sensory and motor aspects of drug cues and drug seeking behavior may modulate or even mediate addictive behavior in addition to the well-studied reward, habit, and inhibitory control systems.

On the other hand, basic cognitive neuroscience research has offered an insight into the mechanisms by which sensory and motor experience alone or in combination with reward-related processes may alter cortical and subcortical representations and shape behavior (Imamizu et al., 2000; Imamizu et al., 2003; Johnson-Frey, 2004; Calvo-Merino et al., 2005; Lacourse et al., 2005; Calvo-Merino et al., 2006; Kourtzi and DiCarlo, 2006; Lewis, 2006; Shuler and Bear, 2006; Graziano and Aflalo, 2007; Weisberg et al., 2007; Kapogiannis et al., 2008; Serences, 2008; Hui et al., 2009). Apparently, intensive long-term sensorimotor experience results in enhanced cortical representations of the respective stimuli and task-related actions. This effect can be further influenced and strengthened by reward associated with the stimuli (Serences, 2008). Furthermore, the efficiency of multisensory integration can be changed by learning and experience (Bavelier and Neville, 2002; D'Ausilio et al., 2006; Ernst, 2007; Musacchia et al., 2007). Since drug cues are often encountered simultaneously in multiple sensory modalities (e.g., 
one sees the cigarette and holds it in the own hand) and addicts have due to their drug taking habits intensive experience with the cues and the corresponding activities, drug addiction seems to offer excellent possibilities for studying the plasticity of multisensory integration in a clinical context.

To sum up, recent findings in addiction cue reactivity research concerning the relevance of sensory and motor processes for cue reactivity imply that there are particular aspects of addiction, which play an important role for its development and maintenance but have not gained the necessary attention in the addiction research community yet. Conversely, while multisensory neuroplasticity research has investigated mainly healthy participants, studying individuals engaging in pathologically compulsive and clinically relevant activities like drug taking and drug seeking behavior, can contribute to a better understanding of the neural mechanisms underlying sensorimotor and multisensory plasticity. Thus, combining basic cognitive with clinical neuroscience may be particularly fruitful: we may gain a novel perspective on some of the mechanisms underlying substance-related addiction. At the same time, basic neuroplasticity research may benefit from findings related to the alterations of sensorimotor and multisensory brain representations occurring after the intensive and often compulsive practice of drug taking behavior.

\section{Aims of the present PhD thesis}

The present $\mathrm{PhD}$ thesis pursues the question of how the combination of these two approaches might be achieved in the context of nicotine addiction. While concentrating on the processes described in the "Models of addiction" section, addiction research has not really considered the possible link between the latest findings of sensorimotor/multisensory neuroplasticity research and the long-term alterations of the human brain due to prolonged drug use behavior. I hypothesize that drug seeking and drug taking behavior is similar to "normal" daily activities in at least one way: prolonged sensory and motor experience strengthens the brain representations of the corresponding objects and actions involved in the particular behavior. Moreover, the intensive reinforcing effects of drugs of abuse enhance these neural representations, too. Taking this into consideration and having in mind the findings of basic neuroscience research showing that activity in sensory, motor and multisensory brain areas can be modified by experience, one should expect that drug cue reactivity involves not only reward, habit and cognitive control systems but also sensorimotor and multisensory systems. Furthermore, because drug paraphernalia are experienced in everyday life not only in the visual but also in other sensory modalities, the type of sensory modality in which the cues are presented during an experiment might modulate the degree to which the reward, habit and cognitive control systems are involved in neural cue reactivity.

To study these questions in terms of cue reactivity in smokers and nonsmokers, we employed functional magnetic resonance imaging (fMRI), which allows to analyze indirectly the neuronal activity in the human brain via measuring the blood-oxygen-level-dependent (BOLD) signal in relation to perceptual or cognitive tasks. A further tool, which we implemented in one of our experiments, is a behavioral task based on the orientation affordance paradigm (Tucker and Ellis, 
1998; Symes et al., 2007; Vainio et al., 2007; Riggio et al., 2008) and modified for the purpose of studying automatized smoking-related reactions. The Fagerström Test for Nicotine Dependence [FTND, (Bleich et al., 2002)], a short questionnaire measuring the severity of nicotine dependence, served to assess the degree of nicotine addiction in smokers.

In Chapter 2 I provide an overview of selected studies stressing the importance of sensory and motor processes for the understanding of the aetiology and symptoms of addiction. On the basis of the reported findings, I propose a model of how sensory and motor processes are involved in the different stages of addiction and how they contribute to the prominent symptoms of the disease.

In Chapter $3 \mathrm{I}$ present an investigation of brain responses to visual smoking-related and control cues in smokers and non-smokers. I focused on the fMRI signal in sensorimotor brain regions, which have been associated with action knowledge and tool use skills. Analyzing the link between fMRI smoking cue reactivity and the degree of nicotine dependence in smokers provides important clinical information on the relevance of sensorimotor neuroplasticity for nicotine addiction. Additionally, we employed a modified version of an established behavioral task to explore how visual smoking cues can trigger smoking-related automatized action schemata in nicotine addicts and to relate these findings to our fMRI results.

Chapter 4 deals with the question whether looking at other sensory modalities than vision would lead to a different neural cue reactivity pattern in smokers. While most neuroimaging studies have used visual drug cues, there has been a remarkable inconsistency in detecting particular brain regions as parts of the brain network related to addiction. Furthermore, to our best knowledge, there is no robust empirical evidence that one sensory modality for cue presentation is more efficient than others in activating the brain regions typically involved in addiction. Therefore, we used fMRI to test whether haptic smoking-cues would induce different neural cue reactivity patterns in smokers than the traditionally used visual cues. Implications of our findings for the development of cue-exposure therapies for addiction and the design of addiction-related experiments are discussed.

Chapter 5 elucidates how visuo-haptic integration of smoking paraphernalia as measured by fMRI differs among smokers and non-smokers and in how far it depends on the individual level of nicotine addiction. The mechanisms of smoking-specific multisensory integration in nicotine addiction and the possibility of studying multisensory neuroplasticity by investigating substance-related addiction are outlined.

In Chapter 6 a brief overview of the main findings of the previous chapters as well as a general discussion of their relevance are provided. Additionally, on the basis of the present $\mathrm{PhD}$ thesis and the reported results, I discuss several issues of crucial interest for the cognitive neuroscience of addiction and multisensory integration which should be considered and implemented in future research and clinical practice. 



\section{Chapter 2}

\section{Sensory and motor aspects of addiction}

\section{Based on:}

Yalachkov Y, Kaiser J, Naumer MJ. (2010) Sensory and motor aspects of addiction. Behavioural Brain Research, 207(2): 215-22. 


\begin{abstract}
Research on the psychological and neuronal underpinnings of addiction has concentrated mostly on affective, motivational, learning, and executive processes and the brain regions subserving these functions. In contrast, sensory and motor aspects of addiction have largely been neglected even though they may be highly relevant for the development and preservation of addiction. The aim of the present chapter is to emphasize the significance of sensory and motor processes for the better understanding and treatment of addiction. We focus on research investigating substance-related addictions and on brain imaging studies in humans that have assessed the contribution of cortical and cerebellar systems to sensorimotor mechanisms of addiction. Activations of sensory and motor brain regions in response to drug-associated cues can predict relapse and correlate with craving, severity of dependence and automatized behavioral reactions towards drug-related stimuli. Furthermore, we propose a model of how sensory and motor processes might be involved in the different stages of addiction.
\end{abstract}




\section{Introduction}

Substance abuse and dependence cause a heavy burden to individuals and societies throughout the world. The World Health Report 2002 indicated that $8.9 \%$ of the total burden of disease is caused by the use of psychoactive substances (WHO, 2002). Particularly important aspects of substance addiction are the high relapse rates which are observed even when well-established pharmacological and psychotherapeutical interventions are applied (Gossop et al., 2002; Piasecki, 2006). In search of explanations for the mechanisms contributing to relapse, different models of addiction have been proposed. Several of these models implicate neurobiological and neuropsychological processes which recently have gained a particular importance in addiction research. Their role for the understanding of addiction has been considered crucial, e.g., with regard to compulsive consumption and relapse after a prolonged time of abstinence. So far, neuroimaging studies of addiction have mostly concentrated on particular brain regions such as the ventral and dorsal striatum, prefrontal cortex, insula, amygdala, hippocampus and anterior cingulate cortex. The psychological processes in the focus of addiction research have been incentive salience, stimulus-response habits, attentional bias, craving, executive functioning and place- and emotional conditioning, as reviewed by Everitt \& Robbins (Everitt and Robbins, 2005). In contrast, the role of sensory and motor processes seems to have been neglected in research on the neurobiological and cognitive correlates of addiction. However, their functional relevance should not be underestimated, as for example activations of sensory and motor brain regions in response to drug-associated cues can predict relapse (Kosten et al., 2006) and correlate with craving, severity of dependence and automatized motor responses to the cues (Smolka et al., 2006; Yalachkov et al., 2009). Although similar findings have been reported in several studies (Brody et al., 2007; Park et al., 2007), they have not received much attention. A review of the corresponding literature is still lacking and the particular role of sensorimotor processes and brain regions in addiction has not been suggested yet.

The aim of the present review is to highlight the potential significance of sensory and motor processes for the understanding and treatment of addiction. We limit this overview to substance-related addictions which have been more intensively studied than non-substance-related addictions. Furthermore, we focus on brain imaging studies in humans. In addition, findings from animal studies are also reported if they contribute to the interpretation of human neuroimaging research. As many of the sensory and motor functions which might be important for addiction are processed mainly in the cerebral cortex and cerebellum, we focus on findings regarding these two parts of the brain. Of course, other subcortical structures such as the basal ganglia or the thalamus might be relevant for this topic, too. However, it is unclear whether activations in these regions reflect predominantly motivational and reward-related or sensorimotor processes. As the answer to this question lies beyond the scope of this review, we only report some findings of particular relevance to the sensorimotor issues of addiction without attempting to offer an exhaustive overview of the role of subcortical structures for addiction. 


\section{Involvement of sensory and motor brain regions in addiction}

\section{Neural cue reactivity in early sensory cortices}

Traditionally, the functions of low-level sensory cortices have been viewed as mainly related to stimulus processing independently of their semantic category, learning history or motivational value. However, nowadays it is well-known that responses in early visual cortices may be influenced by the reward history of the stimuli (Serences, 2008). This explains why these low-level sensory cortices may be relevant for cue-exposure paradigms in addiction research. Several studies have reported robust findings in these regions. Exposure to drug-related visual cues as compared to control stimuli has been shown to induce higher brain activations in addicts in early visual regions like Brodmann areas 17/18/19 (medial occipital lobe, lingual gyrus, lateral occipital gyrus, cuneus) (David et al., 2005; Kosten et al., 2006; Smolka et al., 2006; Brody et al., 2007; McClernon et al., 2007; Park et al., 2007; McClernon et al., 2009; Yalachkov et al., 2009), see Figure 1. Furthermore, this effect was not observed in healthy participants (Yalachkov et al., 2009) and was shown to be correlated with craving (Smolka et al., 2006; Park et al., 2007; Yalachkov et al., 2009), severity of dependence (Smolka et al., 2006; Yalachkov et al., 2009) and treatment effectiveness scores (Kosten et al., 2006). This is rather astonishing because these regions are predominantly involved in the processing of basic visual information and the analysis of rather basic object features. However, these processes can be greatly influenced by the learning history of the stimuli, as some recent studies have shown. Serences (Serences, 2008) demonstrated that spatially selective areas of both primary and higher visual cortices exhibited valuebased modulations of their activity. These value-related influences on the activity of the visual cortex were clearly associated with the reward history of the stimuli. In a reaction time-based task stimuli related to a higher reward value were preferentially selected by the participants and evoked higher haemodynamic responses in spatially selective areas in early visual cortex (V1 but also V2 and V4) and in the intraparietal sulcus and frontal eye fields (FEF).

Further evidence from studies on higher visual areas in the parietal cortex also supports the notion that the reward history of stimuli can affect the response of the visual system in a behaviorally relevant manner (Dorris and Glimcher, 2004; Sugrue et al., 2005). Similar effects have been observed in animal experiments: Shuler \& Bear (Shuler and Bear, 2006) have demonstrated that an association between visual stimuli and subsequent rewards resulted in a new activity pattern of neurons in rat primary visual cortex (V1). Some of the studied V1 neurons began to predict accurately the timing of reward by responding to the relevant visual cue in a manner reflecting the delivery time of the associated reward.

These observations may help to explain the findings concerning early visual circuits and exposure-induced neural reactivity in addicts. One possibility is that the response of the visual system in addicts to drug-related stimuli is stronger than to control stimuli because of their reward history. Environmental stimuli, which appear in a close spatial and temporal relationship to drug consumption, gain incentive salience through associative learning (Robinson and Berridge, 1993, 2001, 2008). The increased salience has been proposed to reflect the neuroplasticity of the reward 

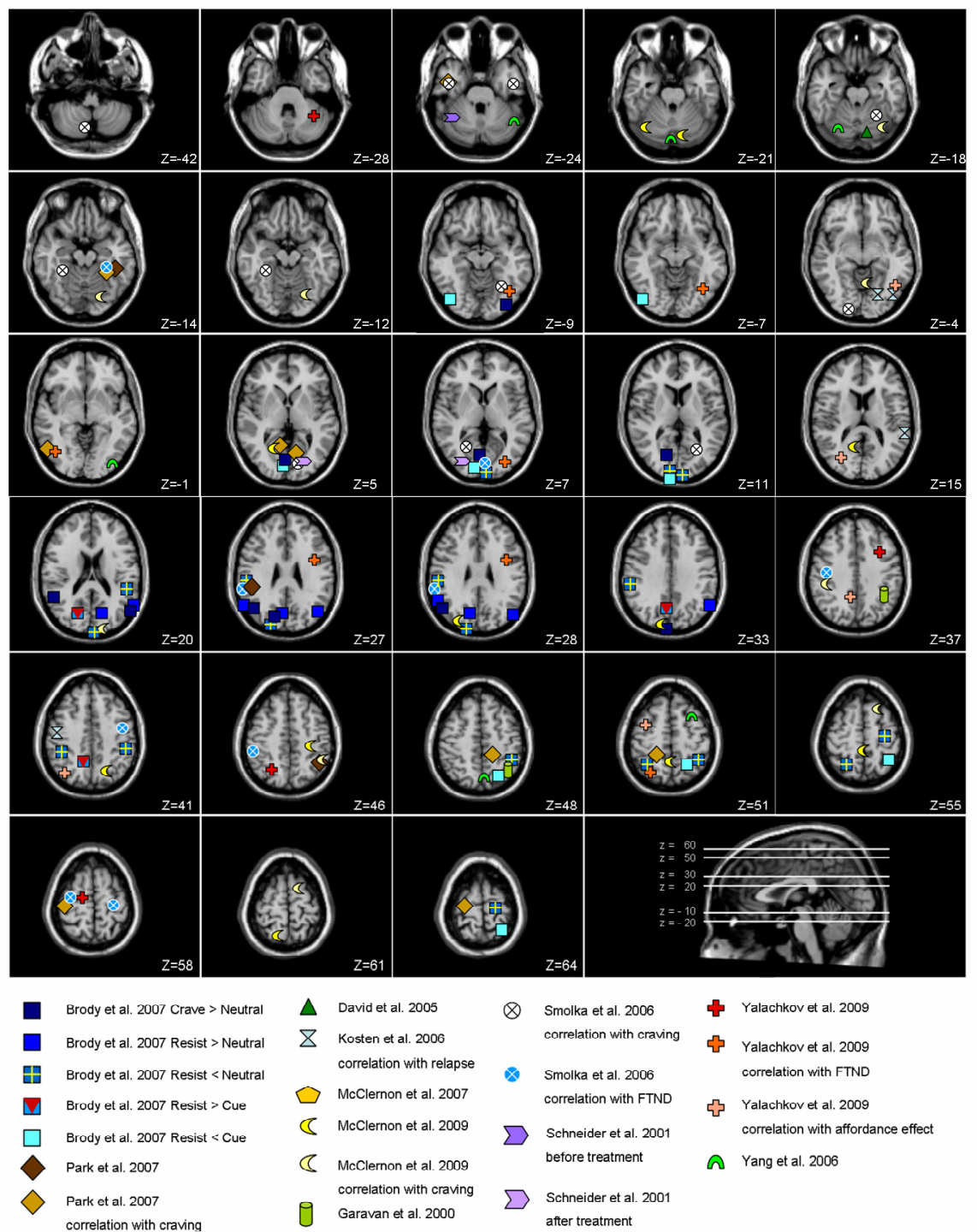

(8) Smolka et al. 2006 correlation with craving

Q Smolka et al. 2006 correlation with FTND

$\sum$ Schneider et al. 2001 before treatment

\& Yalachkov et al. 2009 correlation with FTND

फ Yalachkovet al. 2009 correlation with affordance effect

(A) Yang et al. 2006

Figure 1. Summary of findings of human brain imaging studies of addiction showing activations of sensory and/or motor regions. The most important findings of the reviewed studies (see text for more details) are projected on transversal slices of the MNI template brain transformed into Talairach space (Talairach coordinates in the z-dimension are specified for a better orientation, see bottom right image). The legend below the figure provides additional information on whether the findings refer to correlations of brain activations with subjective craving, FTND scores reflecting the severity of nicotine dependence, affordance effects (i.e., reflecting automatized behavioral reactions towards drug-related stimuli) or indicators of relapse. If nothing else is indicated, the effects were obtained by comparison between brain activations in substance-dependent or misusing patients induced by substance-related cues versus control cues and/or healthy control participants. In the study of Brody et al. (Brody et al., 2007) "crave" stands for the experimental condition where the participants were exposed to substance-related cues and craving was allowed; "resist" indicates the condition, where they were instructed to suppress craving when exposed to the cues; "neutral" means confrontation with neutral stimuli. 
system. It is conceivable that this also applies to the early stages of processing of drug-related visual information, thus creating not only a sensory, but also a behavioral bias, as Serences was able to demonstrate (Serences, 2008). Most interestingly, this hypothesis has been supported by complementary investigations of early auditory and somatosensory areas. For example learning-induced and rewardor punishment-mediated representational expansions have been observed in the primary auditory cortex of both rats and humans (Bakin and Weinberger, 1990; Morris et al., 1998; Gao and Suga, 2000; Rutkowski and Weinberger, 2005; Weinberger, 2007; Hui et al., 2009).

The tuning of neurons in primary auditory cortex has typically been found to shift towards the frequency of a tone that has served as a signal for reward or punishment. Furthermore, control of behavior by conditioned tones has been observed only in animals showing plasticity of the auditory cortex (Hui et al., 2009). Apparently, neuronal plasticity in early sensory cortex serves to increase the representation of a behaviorally relevant sound. Chaudhri et al. have shown how this process can be linked to addictive disorders: a conditioned auditory stimulus that has been paired with ethanol could trigger a relapse in trained rats even after a number of extinction sessions (Chaudhri et al., 2008). Unfortunately, only few studies exist on exposure to auditory drug-related cues in human addicts. Heinze et al. studied the effects of alcohol-associated auditory stimuli on alcohol craving (Heinze et al., 2007). They showed that the enhanced motivational response to the alcohol-related stimuli was correlated with higher amplitudes of the P300 event-related potential (ERP) in detoxified alcoholics compared to healthy controls. However, further research is needed to investigate whether drug-related acoustic cues can elicit enhanced activations in early auditory regions as reflected by early ERP components generated in superior temporal cortex.

Similar findings suggest that activity in the somatosensory system can also be modulated by reward history. Pleger et al. found that rewards for the correct discrimination of electrical somatosensory stimuli not only improved performance but also reactivated the primary somatosensory cortex contralateral to the stimulated hand in the absence of somatosensory input (Pleger et al., 2008). Moreover, stronger reactivations were observed for higher reward values, and the level of reward influenced performance and functional magnetic resonance imaging (fMRI) activity in the subsequent trial. Pantoja et al. revealed that stimulus-related information in primary somatosensory cortex (S1) and the ventral posterior medial nucleus of the thalamus varied with the reward contingency of the task when rats were required to associate two different tactile stimuli with two corresponding choices of spatial trajectory (Pantoja et al., 2007). Moreover, S1 activity strongly correlated with task performance long after stimulus removal. Although human studies where drugrelated stimuli are presented only in the haptic modality are still lacking, there is evidence that the somatosensory system may be relevant for addiction research, as exposure to visual drug-related cues results in activation of early somatosensory regions (Smolka et al., 2006; Brody et al., 2007; McClernon et al., 2009). It seems that somatosensory circuits can be influenced by the reward effects of drugs and that later exposure to associated visual stimuli may activate not only sensory representations in the visual, but also in the haptic modality. Indeed, some studies reveal that the acute rewarding effects of drugs of abuse like cocaine can enhance cortical processing of somatosensory stimuli. Devonshire et al. showed that 
haemodynamic responses to sensory stimulation as well as field potentials, current source density and multi-unit activity in response to stimulation were enhanced in the primary sensory (barrel) cortex of rats following acute cocaine administration (Devonshire et al., 2004; Devonshire et al., 2007). More specifically, while reductions in response magnitude with repeated sensory stimulation were observed following saline injection, this sensory adaptation was reduced after cocaine administration (Devonshire et al., 2007). Importantly, these effects were found in layers 2 and 3 of the barrel cortex, which have been related to experience-based plasticity (Huang et al., 1998; Fox, 2002; Hickmott and Merzenich, 2002; Petersen, 2007). An enhanced initial cortical processing of environmental somatosensory stimuli may contribute to the formation of their high motivational values. Thus, the acute effects of cocaine may participate in the formation of particularly strong salience of drug-related cues and make them more attractive and behaviorally relevant. Studies on human addiction have used visual, auditory or olfactory, but rarely somatosensory cues for exposure paradigms. Addiction studies in the haptic modality may contribute to a better understanding of the early sensory processing in addiction.

Attentional bias is another factor which might be linked to the preferential processing of drug-related cues in early sensory cortices. Increased attention has been shown to alter activity in early sensory cortical regions (Kastner and Ungerleider, 2000). Drug-related cues may attract the attention of addicts more strongly than control stimuli and thereby induce higher activations in early visual circuits. Several studies have shown that attentional bias in substance abusers can be found during rather short temporal intervals $(50-100 \mathrm{~ms})$ after the presentation of the substance-related stimuli (Stormark et al., 1997; Noel et al., 2006). Moreover, substantial evidence points to the importance of attentional bias for addictive behaviors (Franken, 2003; Field, 2006a; Robbins et al., 2008). It has been suggested that exposure to substance-related stimuli can result in both attentional bias and subjective craving which are both mediated by the expectancy of substance availability. Furthermore, attentional bias and craving seem interrelated with an increasing bias leading to more craving and vice versa [for a review see (Field and Cox, 2008)]. This could explain why the cue-exposure effects observed in visual cortices are correlated with measures of craving (Smolka et al., 2006; Park et al., 2007). Further factors seem to modulate these effects. Severity of nicotine dependence was found to be correlated with the preferential activation of visual cortical regions by smoking-related cues as compared to control cues (Smolka et al., 2006; Yalachkov et al., 2009), indicating that heavier smokers may be affected more strongly by the mechanisms of attentional bias and/or reward-dependent modulation of visual stimulus processing. Most interestingly, there is evidence that the effects in the early visual circuits can be influenced by cognitive control strategies. Brody et al. showed that when smokers were asked to suppress their craving during exposure to smoking-related cues, the activity in the cuneus and lateral occipital gyrus decreased in comparison to neutral or "craving allowed" conditions (Brody et al., 2007). This confirms the predictions of Field \& Cox (Field and Cox, 2008) who have postulated that cognitive avoidance strategies influence the strength of attentional bias and subjective craving, particularly when the substance-related cues are presented long enough to allow the participants to recruit cognitive control mechanisms. 
Finally, early sensory cortex responses seem to be relevant for the treatment of addiction. Kosten et al. have demonstrated that the fMRI signal in the right lingual and inferior occipital gyrus (BA19) in response to cocaine-related cues could predict the treatment effectiveness score, serving to identify patients who were more vulnerable to drop-out and/or relapse during treatment (Kosten et al., 2006).

\section{Object recognition in addiction}

It is well-known that the primate visual system involves hierarchical processing (Felleman and Van Essen, 1991). Thus visual information which has been processed in the early visual cortices is transferred to higher visual brain regions such as the inferior temporal cortex (ITC) and fusiform gyrus (FG). These regions have been implicated in the perception and recognition of complex visual stimuli (Ungerleider and Mishkin, 1982; Goodale and Milner, 1992; Logothetis and Sheinberg, 1996). Furthermore, neuropsychological and neuroimaging work suggests that these regions are involved in the representation of category-specific information.

These regions also seem to be involved in the neural cue reactivity in addiction. Higher fMRI responses for substance-related than for control stimuli were found in the FG and in the ITC by several studies (Due et al., 2002; David et al., 2005; Smolka et al., 2006; Park et al., 2007; McClernon et al., 2009; Yalachkov et al., 2009; Yang et al., 2009), see Fig.1. The implications of these findings become clearer, when considering the functional significance of these regions in the healthy brain. The ITC and FG are mainly associated with identifying complex stimuli and classifying them into a limited number of semantic categories such as living vs. nonliving things, faces, body parts, etc. (Gross, 1992; Tsao et al., 2003; Kanwisher and Yovel, 2006; Martin, 2007; Kriegeskorte et al., 2008; Bell et al., 2009). Relating a perceived stimulus to a particular category and accessing the corresponding semantic knowledge is crucial for real-world experience. Also from an evolutionary perspective it is important to judge whether an object is something edible, whether it can be used as a tool, or whether it represents a danger. Of course, visual perception does not involve the fully conscious and elaborate processing of these questions. Yet we identify the perceived object as a member of a particular category almost instantaneously and without effort. This implies that the involvement of the ITC and FG occurs nearly automatically. Importantly, the addiction studies reported above used cue reactivity paradigms consisting of confrontation of addicted and control subjects with substance-related stimuli. It seems reasonable to expect that addicted participants recruit efficiently the ITC and FG in object recognition and classification processes during the exposure to the substance-related visual cues. This facilitates the perception of the stimuli as drug-associated and their recognition as relevant to their addiction. Moreover, several studies have shown that neural representations of object categories in the ITC and FG are plastic and can be altered by intensive visual and/or functional experience (Gauthier et al., 1999; Op de Beeck et al., 2006; Weisberg et al., 2007; van der Linden et al., 2008). This could explain why the degree of drug dependence was found to correlate with fMRI activation in FG and ITC for substance-related as compared to control stimuli (Smolka et al., 2006; Yalachkov et al., 2009). Addicts may acquire an object-recognition and classification expertise with regard to images associated with their addiction. Thus, an efficient access to the semantic knowledge of addiction-related paraphernalia 
may prepare the patient for further, more elaborate processing of the substance cues and trigger mechanisms of relapse. This hypothesis is supported by a recent study showing that a measure of automatized motor reactions towards smoking-related paraphernalia was positively correlated with the preferential processing of smokingrelated cues in the ITC of smokers (Yalachkov et al., 2009). Further investigation of this phenomenon and direct testing of the hypothesis postulated above seem to be necessary to fully disentangle the mechanisms underlying the neural cue reactivity patterns in ITC and FG in addicts.

\section{Involvement of visuomotor brain regions in addiction}

Other brain regions involved in neural cue reactivity in addiction, include the supramarginal gyrus, precuneus, inferior and superior parietal lobules (see Fig. 1). The involvement of the supramarginal gyrus was demonstrated in (Brody et al., 2007), who observed higher fMRI responses for smoking-related stimuli in comparison to control cues. This difference remained stable even when the participants were instructed to suppress their craving for nicotine. A study by Russ et al. indicated that the supramarginal gyrus is crucial for the retrieval of memories for enacted actions (Russ et al., 2003). On the basis of a literature review Hackley suggested that the supramarginal gyrus may be involved in the lowering of the threshold for response selection in healthy participants (Hackley, 2009).

Preferential activation of the precuneus for substance-related compared with control stimuli has been demonstrated by several studies (Brody et al., 2007; Yalachkov et al., 2009; Yang et al., 2009). Moreover, reaction time advantages for automatized motor responses to the addiction-relevant stimuli in smokers were found to be correlated with this effect (Yalachkov et al., 2009). This is compatible with the suggested role of the precuneus for motor imagery and mental simulation of locomotor tasks (Ogiso et al., 2000; Malouin et al., 2003).

The inferior and superior parietal lobules also seem to participate in the neural cue reactivity in addiction (Garavan et al., 2000). These two brain regions exhibit not only higher fMRI signals to substance-related than to control cues, but also a correlation of this effect with the degree of dependence (Smolka et al., 2006; Yalachkov et al., 2009) or subjective craving (Park et al., 2007). Some of the suggested functions of these regions are the integration of sensory and motor signals, the processing of spatial representations for reaching and grasping of objects and sensory guidance of movements (Fogassi et al., 2005; Jeannerod and Jacob, 2005; Buneo and Andersen, 2006). Thus, these regions seem to link perception and action (Fogassi et al., 2005).

The evidence reported above underscores the significance of the supramarginal gyrus, precuneus, and both inferior and superior parietal lobules for cue reactivity in addiction. Apparently, substance-related cues are able to activate visuomotor brain regions more strongly than control stimuli. This might reflect imagery and/or preparation of approach-related actions towards the substanceassociated stimuli. In addition, the modulation of cue reactivity by the severity of dependence yielded evidence for a clinically relevant involvement of the brain regions listed above. 


\section{The role of motor brain regions in addiction}

Until recently the motor aspects of addiction have been studied mostly with regard to locomotor sensitization, i.e. increased locomotor activity after repeated drug administration which has been linked to the dopamine pathways projecting from the ventral tegmental area to the NAcc (Robinson and Berridge, 1993; Vezina et al., 2002; Vezina, 2004). However, some studies have shown that another aspect of motor behavior, i.e. automatized motor skills, may play an important role in addiction.

The formation of motor skills in addiction can apparently be modulated by the acute effects of cocaine (Willuhn et al., 2003; Willuhn and Steiner, 2006, 2009). Willuhn et al. have demonstrated that cocaine not only induces molecular adaptations preferentially in cortico-basal ganglia circuits receiving inputs from sensorimotor cortex through caudal dorsal striatum but also that in rats some of these changes can be influenced by the behavior performed during drug exposure (Willuhn et al., 2003). A further study showed that wheel training under cocaine altered gene expression in rat striatum (Willuhn and Steiner, 2006). Moreover, these changes went along with improvements of the wheel running skill, demonstrating a facilitating effect of cocaine on motor training. The formation of long-term skill memory can be disrupted by intrastriatal infusion of lidocaine (Willuhn and Steiner, 2009). However, pre-trial cocaine administration prevented the disruption of skill consolidation, indicating that it can facilitate motor memory formation and prevent it from disturbing environmental influences. It seems that cocaine supports the development of motor skills, which are spatially and temporally related to drug administration. These drug-associated motor skills may correspond to the automatized drug-related skills in humans which develop through intensive and regular practice in the course of addiction.

In general, automatic behavioral responses can be considered as consisting of two different processes, the first one triggering the response and the second one controlling the performance of this response (Belin et al., 2009). The first aspect has gained particular importance in the field of addiction research and is related to the habitual or stimulus-response approach. Everitt and Robbins (Everitt and Robbins, 2005) and others have already shown that habitual mechanisms encoded by the dorsolateral striatum gain behavioral control during the course of addiction. Habitual stimulus-response mechanisms have been proposed to develop mainly due to excessive, long-term instrumental conditioning processes (Everitt and Robbins, 2005; Robbins et al., 2008). This aspect is indisputably of great importance for compulsive drug seeking and drug taking. However, there is a second aspect of automaticity in addiction, which also seems to be of importance for drug consumption and relapse but has been neglected by current addiction research. This aspect of automatic behavioral responses refers to procedural memory processes in terms of object manipulation and motor skills which evolve from intensive and repetitive motor training. The automatized action skills contribute to phenomena as increased performance, faster responses and subjective perception of tasks as less demanding (Schneider and Shiffrin, 1977; Shiffrin and Schneider, 1977, 1984; Logan, 1988). We suggest that these drug-associated sensorimotor skills also exert an influence on the addict's behavior and are complementary to the well-established habitual mechanisms proposed by Everitt and Robbins (Everitt and Robbins, 2005). 
Importantly, the development of these skills may be facilitated by the acute effects of some drugs (e.g., cocaine), as illustrated by the studies described above. Deeply embedded motor and drug paraphernalia manipulation skills are believed to be of great importance for automatized action representations in addicts (Tiffany, 1990) and recent evidence from neuroimaging and behavioral studies has supported this statement and shown a link between severity of dependence, automatized motor responses towards drug-related cues and neural correlates of object manipulation skills (Yalachkov et al., 2009).

The review of the literature on action representation, motor skills, object manipulation knowledge and their automatization reveals that these functions typically involve the motor and premotor cortex, supplementary motor area and cerebellum (Fink et al., 1999; Arbib et al., 2000; Lang and Bastian, 2002; Grezes et al., 2003; Imamizu et al., 2003; Creem-Regehr and Lee, 2005; Monfils et al., 2005; Poldrack et al., 2005; Grol et al., 2006; Higuchi et al., 2007; Weisberg et al., 2007). Surprisingly, we found that a large number of studies using the cue reactivity paradigm have reported preferential activations of these regions for substancerelated stimuli in addicts in comparison to control cues and/or groups (Schneider et al., 2001; Kosten et al., 2006; Smolka et al., 2006; Brody et al., 2007; McClernon et al., 2009; Yalachkov et al., 2009; Yang et al., 2009), see Fig. 1. Due to the rapidly growing research on experience-based neural plasticity it is well-known that intensive and frequent engagement in an activity leads to stronger neural representations of the respective activity and the used objects (Pearce et al., 2000; Sanes and Donoghue, 2000; Calvo-Merino et al., 2006; Schwenkreis et al., 2007; Weisberg et al., 2007). Thus, confrontation with drug-related cues on a regular basis and intensive practicing of drug consumption behaviors may facilitate the development and automatization of deeply embedded action schemata as postulated by Tiffany (Tiffany, 1990). From a cognitive point of view these automatized action schemata contain information underlying the efficient initiation and execution of drug taking behavior (Tiffany, 1990). They can be triggered rapidly by drug-related cues, thus increasing the probability that addicts will perform substance-use actions independently of intentional processes. Indeed, substance consumption (e.g., smoking) has been shown to be more automatized in heavier users compared to novices in terms of cognitive load and dual task performance during smoking (Baxter and Hinson, 2001; Field et al., 2006b). Recently, we have shown that smoking-related motor representations could be activated in smokers but not in nonsmokers as measured by a behavioral orientation affordance paradigm and that this effect correlated strongly with both the individual degree of nicotine dependence and with the fMRI cue reactivity in smokers in regions forming part of the brain network related to motor representation and action knowledge (Yalachkov et al., 2009). Other groups have also found correlations between the activation of these brain regions and the severity of dependence or subjective craving (Smolka et al., 2006; Park et al., 2007; McClernon et al., 2009). The finding that suppressing craving for smoking induces deactivation of the motor cortex in smokers seems to be particularly important (Brody et al., 2007). Similarly relevant is the observation that the fMRI cue reactivity signal from the motor cortex can predict the degree of effectiveness of the treatment in cocaine addicts (Kosten et al., 2006). Most importantly, the neural cue reactivity pattern of some of these brain regions can be altered by therapeutical interventions. Schneider et al. (Schneider et al., 2001) have 
reported that cerebellar activations in detoxified alcoholics in response to olfactory stimulation could be observed before but not after three weeks of a behavioral therapy with psychopharmacological intervention. This should encourage addiction researchers to further investigate the neural correlates of automatized substancerelated action schemata and motor knowledge, to elucidate their involvement in relapse and to reveal how they can be modified by therapeutical approaches.

There is some evidence that additional brain regions may be involved in the motor preparation of substance-related actions. Gloria et al. (Gloria et al., 2009) revealed significant cue-induced effects in smokers in a withdrawal state in the caudate and in the ventral anterior nucleus of the thalamus in response to a nicotine preinfusion warning cue. Based on findings of other research groups reporting an involvement of the thalamus and the caudate nucleus in motor functions, they argued that drug anticipation can serve as a conditioned stimulus triggering motor programs related to drug administration. Future research may help elucidating the exact role of these structures in addiction-related motor programs, as thalamus and caudate nucleus have also been implicated in other perceptual, cognitive and motivational functions like, e.g., the integration of sensory information, or the development of habits through intensive instrumental conditioning.

\section{A model of sensory and motor contributions to the development of addiction and mechanisms of relapse}

In the last part of the present review we propose a model describing how sensory and motor processes might be involved in different stages and mechanisms of addiction (Figure 2). On the basis of what we know from animal experiments (Willuhn et al., 2003; Devonshire et al., 2004; Willuhn and Steiner, 2006; Devonshire et al., 2007; Willuhn and Steiner, 2009), we suggest that some of the drugs of abuse (particularly cocaine) can enhance sensory processing via its acute effects and thus facilitate cortical learning and plasticity processes. This can be crucial for the development of strongly embedded sensory representations, as at this stage drug-related environmental cues are processed by the brain very effectively. Furthermore, cocaine can facilitate the development of drug-associated motor skills, supporting brain circuits responsible for developing motor programs as well as protecting them from disturbing environmental influences.

The transition from initial to advanced stages of addiction is marked by the development of value- and experience-based sensory and motor representations. Drug cues acquire motivational values dependent on their reward history. This process is reflected not only by the reward system but also by the sensory and perceptual systems. Moreover, intensive and regular involvement in activities with the drug and the corresponding paraphernalia results in deeply embedded action schemata which contain motor knowledge of substance acquisition and consumption actions that are quickly accessible and can be easily triggered.

Exposure to substance-related cues in the later, advanced stages of addiction activates the respective sensory and motor representations. Thus, drugassociated sensory cues gain increased salience, elicit an attentional bias, and their processing in higher brain regions assures quick and efficient access to the relevant 
semantic knowledge. Importantly, the triggering of automatized action representations may reduce the threshold for preparing the corresponding movement and carrying out drug seeking and drug taking actions.

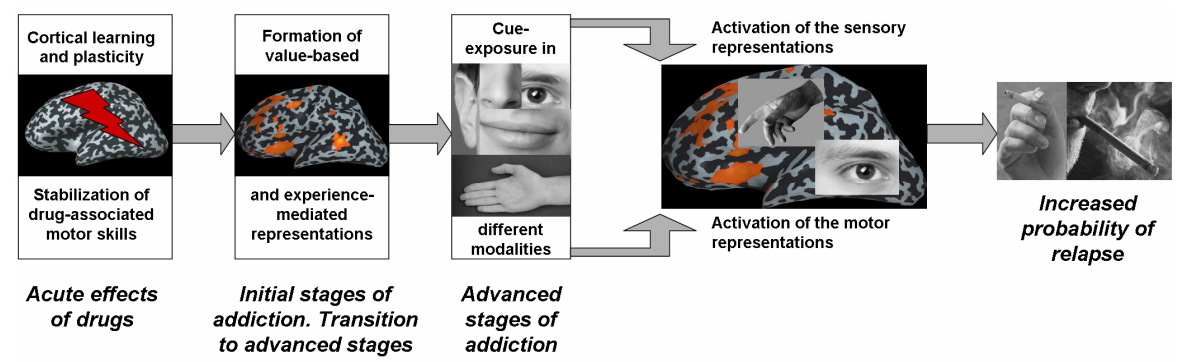

Figure 2. A proposed model for the involvement of sensory and motor mechanisms in different stages of addiction. Sensory and motor processes involve corresponding brain regions differently across the single stages of addiction. Acute drug effects facilitate the development of sensory and motor representations via mechanisms of cortical plasticity and enhanced sensory and motor learning. These representations are further developed and embedded via reward- and experience-dependent learning during the initial and advanced stages of addiction. In advanced stages of addiction, cue exposure can elicit activations of the sensory and motor representations and thus contribute to the increased likelihood of relapse into the drug consumption pattern.

Thus, confrontation with substance-related cues may increase the probability of relapse. Of course, this knowledge is not really new for the research field of addiction. However, the novel aspect is the particular involvement of sensory and motor processes and of brain regions which have been rather neglected by research on addiction and relapse. The present review aimed at providing an overview of the current evidence for sensory and motor aspects of addiction and an appropriate framework for the development of further research. Future studies may test directly some of the statements proposed above and thus contribute to further elucidating the sensory and motor underpinnings of addiction. 



\section{Chapter 3}

\section{Brain regions related to tool use and action}

knowledge reflect nicotine addiction

\section{Based on:}

Yalachkov Y, Kaiser J, Naumer MJ. (2009). Brain regions related to tool use and action knowledge reflect nicotine dependence. Journal of Neuroscience, 29, 4922-4929. 


\begin{abstract}
In addition to reward- and craving-related processes, habitual mechanisms play an important role in addiction. While the dorsal striatum has been proposed to code for the motivational state of habitual drug seeking actions, the neural underpinnings of the corresponding drug taking skills and action knowledge remain poorly understood. We used functional magnetic resonance imaging (fMRI) and a behavioral orientation affordance paradigm to investigate the neural and behavioral correlates of automatized drug taking actions in nicotine dependence. Smokers exhibited higher fMRI activations than non-smokers when viewing smoking-related but not when viewing control images. These group differences in fMRI activations were located not only in brain regions associated with craving and habitual learning (left ventral and dorsal striatum, dorsolateral prefrontal cortex, insula, uncus, medial frontal gyrus, right subcallosal gyrus, and bilateral parahippocampal gyrus), but also in a network of brain regions which has been strongly implicated in the encoding of action knowledge and tool use skills (bilateral premotor cortex, left superior parietal lobule, and right lateral cerebellum). A behavioral affordance reaction-time task indicated that smokers, but not non-smokers, showed an automatized responsiveness to smoking paraphernalia similar to everyday objects. Moreover, smokers showed strong intercorrelations between fMRI activations in tool use-related brain regions, behavioral responsiveness to smoking-related cues, and severity of nicotine dependence. Apparently smoking-related action representations in smokers are stored in brain regions typically representing tool use skills and action knowledge. Most importantly, cortical and behavioral correlates of the respective drug taking skills vary with the individual degree of nicotine dependence.
\end{abstract}




\section{Introduction}

With an estimated death toll of $>5$ million per year (WHO, 2008) nicotine dependence is the addiction with the strongest impact on public health. While sophisticated medical and psychotherapeutic treatments have contributed to a certain increase in abstinence rates, even the most effective approaches have long-term success rates of only $\sim 30 \%$ (Piasecki, 2006). While research has focused mainly on the reward system and craving, there is evidence that relapse into drug taking behavior is not always related to conscious craving (Miller and Gold, 1994). Instead, the high change resistance of smoking behavior might be attributable also to deeply embedded habitual processes, which are compulsive and almost automatic. From a cognitive point of view, these habitual mechanisms are encoded as automatized action schemata, which develop with repeated use and contain information underlying the efficient initiation and execution of drug taking behavior. These schemata can be triggered rapidly by smoking cues, thus increasing the probability that smokers will perform substance-use actions independently of intentional processes. It has been shown that smoking behavior becomes automatized in frequent smokers (Baxter and Hinson, 2001; Field et al., 2006b). Furthermore, recent synaptic plasticity research has identified the dorsal striatum as the region contributing to the establishment of persistent drug-related habits (Gerdeman et al., 2003). While the activation patterns in the dorsal striatum reflect the formation of powerful stimulus-response associations (Everitt and Robbins, 2005), very little is known about the neural substrates of the corresponding drug taking skills and action knowledge.

The present study explored the neural and behavioral correlates of drug taking skills and smoking-related action knowledge in smokers and their associations with the individual degree of nicotine dependence. Smokers and nonsmokers were scanned with functional magnetic resonance imaging (fMRI) while viewing smoking-related and control images. We hypothesized that smokingrelated but not control cues trigger the respective skill and action representations, which should be reflected by higher fMRI activations in smokers compared with nonsmokers in brain regions known to encode tool use skills and action knowledge. Furthermore, as automatized behavioral patterns are executed relatively fast and efficiently once they are triggered (Tiffany, 1990), we expected an increased behavioral responsiveness to smoking-related objects in smokers compared with nonsmokers. To assess behavioral responsiveness to both everyday and smokingrelated objects we used an orientation affordance paradigm. This is a stimulusresponse compatibility paradigm which has been used in numerous studies (Tucker and Ellis, 1998; Symes et al., 2007; Vainio et al., 2007; Riggio et al., 2008) showing that certain action-related properties of a stimulus (e.g., spatial information in terms of left/right orientation) generate automatic response codes (Tucker and Ellis, 1998). Thus, it is a suitable instrument to study the automatic activation of motor representations by graspable tools (Tucker and Ellis, 1998; Vainio et al., 2007). Since the automatization of drug use behaviors increases with repeated practice, action representations on both the neural and behavioral levels should correlate with the individual degree of nicotine dependence. 


\section{Materials and Methods}

\section{fMRI study}

\section{Subjects}

All participants were right handed, had normal or corrected-to-normal vision and were students or university academic staff. The smokers $(n=15$, mean age 27.1 years ( $\mathrm{SD}=3.8), 6$ men and 9 women) were instructed to smoke according to their usual habits. Their individual level of nicotine dependence was evaluated with the Fagerström Test for Nicotine Dependence (FTND) (Bleich et al., 2002). The mean FTND score accounted to $3.13, \mathrm{SD}=2.39$. We recruited light as well as heavy smokers to represent the whole spectrum of smoking behavior. The nonsmokers $[\mathrm{n}=$ 15 , mean age 28.7 years $(\mathrm{SD}=6.8), 6$ men and 9 women] claimed that they had never smoked. After complete description of the study to the subjects, written informed consent was obtained.

\section{Experimental design}

During the experiment a total of 40 grayscale images from different categories were presented to the participants. The first category comprised images of humans smoking cigarettes ("smoke" condition) and the second category consisted of images of humans holding pens or glasses in their hands and mouths ("control" condition). The other three categories (data not reported here) consisted of images of everyday tools and animals and scrambled images. The images of humans were taken from the International Smoking Image Series (Gilbert and Rabinovich, 2006). We ensured that all images had the same size.

The participants underwent four functional runs and one anatomical scan. A dummy block at the beginning of each run ensured that $\mathrm{T} 1$ saturation effects were avoided. The eight images from the five categories were presented in separate blocks. Blocks for each image category were repeated three times within each run in pseudorandomized order, thus resulting in 15 experimental +1 dummy block per run. Blocks lasted for $16 \mathrm{~s}$ and alternated with fixation intervals of equal length. Images were presented in a randomized order for $1500 \mathrm{~ms}$ each with $500 \mathrm{~ms}$ interstimulus intervals in a block. We chose a passive paradigm to avoid confounding task-related sensorimotor activations. The participants were instructed to fixate the white cross and attend the images, which were projected onto a vertical screen positioned inside the scanner. Subjects viewed the screen through a mirror.

The fMRI procedure was performed on a 3-tesla Magnetom Allegra scanner (Siemens) at the Brain Imaging Center in Frankfurt am Main. A gradient-recalled echo-planar imaging sequence with the following parameters was used: 34 slices; repetition time $2000 \mathrm{~ms}$; echo time (TE) $30 \mathrm{~ms}$; field of view $192 \mathrm{~mm}$; in-plane resolution $3.3 \mathrm{~mm} 2$; slice thickness $3 \mathrm{~mm}$; gap thickness $0.3 \mathrm{~mm}$; 264 volumes per functional run. For the anatomical imaging a magnetization-prepared rapidacquisition gradient echo sequence (TR $2300 \mathrm{~ms}$, TE $3.49 \mathrm{~ms}$, flip angle $12^{\circ}$, matrix $256 \times 256$, and voxel size $1.0 \times 1.0 \times 1.0 \mathrm{~mm} 3$ ) was used. 


\section{Statistical analysis}

Data were analyzed using the BrainVoyager QX 1.10.4 software package (Brain Innovation). Preprocessing included the following steps: three-dimensional motion correction, linear-trend removal and temporal high-pass filtering at $0.0054 \mathrm{~Hz}$, and slice-scan-time correction with sinc interpolation. Functional and Talairachstandardized anatomical data were used to build a voxel time course file and were spatially smoothed with a Gaussian filter of $8 \mathrm{~mm}$ full width at half maximum. Volume-based statistical analyses were performed at the whole-brain level. For each voxel, the time course was regressed on a set of dummy-coded predictors representing the experimental conditions. To account for the shape and delay of the haemodynamic response, the predictor time courses (boxcar functions) were convolved with a gamma function.

First, a two-way random-effects analysis (RFX) ANOVA with factor 1 "stimulus category" (smoke vs. control) and factor 2 "group" (smokers vs. nonsmokers) was calculated ( $\mathrm{n}=30$ [15 smokers, 15 nonsmokers]). Then, a wholebrain statistical map for the interaction "stimulus category" $\mathrm{x}$ "group" was computed. Thus, we were able to show which regions exhibited differential fMRI activations across groups and object categories. Further analysis of these regions was performed with a ROI-based random-effects general linear model to identify the group/object differences which had caused the significant RFX ANOVA interaction. Unpaired $t$ tests of the estimated $\beta$-values for the two groups in the smoke and control conditions were performed to reveal the regions in which the fMRI activation during presentations of smoking-related objects was higher in smokers than nonsmokers.

For the analysis of correlations between fMRI cue reactivity and the individual degree of nicotine dependence a whole-brain RFX ANCOVA with the contrast "smoke > control" and the covariate "FTND scores" was computed $(n=15$ smokers, "RFX ANCOVA-FTND"). The resulting statistical map showed the regions where the $\beta$ weights of the GLM-contrast "smoke > control" and the individual FTND scores correlated significantly.

A similar procedure was used for correlations between fMRI cue reactivity and the behavioral task performance (see below). A whole-brain RFX ANCOVA with the contrast "smoke > control" and the covariate "affordance index for smoking objects" was computed ( $n=8$ smokers; "RFX ANCOVA-affordance"). The resulting statistical map showed the regions where the $\beta$ weights of the GLMcontrast "smoke > control" and the individual affordance indices for smoking objects correlated significantly.

The RFX ANCOVA-FTND was computed with the data of 15 participants who passively viewed smoking-associated and control images. The RFX ANCOVAaffordance was based on the data of 8 smokers who were involved additionally in the active stimulus-response compatibility task. According to our hypotheses, automatized smoking-related skills and action knowledge should be activated in both cases and may share common neural correlates. However, due to the different experimental designs, we did not expect that the two statistical maps resulting from the RFX ANCOVA-FTND and RFX ANCOVA-affordance would reveal identical regions. We performed a further ROI-based analysis of the two statistical wholebrain maps to assess the similarity of the neural correlates of automatized smokingrelated schemata in the two tasks. In the regions revealed by the whole-brain RFX 
ANCOVA-FTND, a ROI-based RFX ANCOVA with the contrast "smoke > control" and the covariate "affordance index for smoking objects" was performed. Similarly, a ROI-based RFX ANCOVA with the contrast "smoke > control" and the covariate "FTND" was computed in the regions revealed by the whole-brain RFX ANCOVAaffordance. All statistical maps were corrected for multiple comparisons using a cluster-size threshold procedure and projected onto anatomical data sets averaged across all 30 participants.

\section{Behavioral study}

Subjects

We recruited 8 of the smokers [mean age 27.5 years $(\mathrm{SD}=6.09), 3$ men and 5 women; mean FTND score $2.38(\mathrm{SD}=2.67)]$, who had participated in the fMRI experiment and whose FTND scores had remained stable over the time interval between the fMRI and behavioral experiments (on average 5 months), and 8 nonsmokers [mean age 28.13 years $(\mathrm{SD}=3.27), 3$ men and 5 women]. All the participants were right-handed students or university academic staff and had normal or corrected-to-normal vision. The smokers were instructed to smoke according to their usual habit. After complete description of the study to the subjects, written informed consent was obtained.

\section{Experimental design}

The participants sat in a dark, soundproofed room in front of a monitor on which visual stimuli were displayed. The experiment comprised five runs with 112 trials each. Each trial began with a white fixation cross visible for $1500 \mathrm{~ms}$. Afterwards, a randomly chosen object was presented for $700 \mathrm{~ms}$. Then the white fixation cross appeared again over the object and after $150 \mathrm{~ms}$ changed its color randomly from white to blue or brown. The new color was kept for $180 \mathrm{~ms}$ and then the fixation cross turned its color back to white. Half of the participants was instructed to respond with the left control button on the keyboard to a color change from white to blue and with the right control button to a color change from white to brown. The other half of the participants received the inversed mapping instruction.

Grayscale images of objects from two categories were used: (1) everyday tools/manipulable objects (e.g., cup, scissors, hammer, etc.) and (2) smoking-related objects (e.g., cigarette, lighter, etc.). Images originated from commercially available image collections (150.000 PhotoObjects Graphics Collection and The Big Box of Art 615 000, Hemera Technologies). We ensured that all images had the same size. Each of the objects was presented with its handle or side where the object is usually grasped in left or right orientation. Thus, we had a $2 \times 2 \times 2 \times 2$ experimental design (within-subjects factors: object category, responding hand, object orientation; between-subjects factor: smokers vs nonsmokers). Seventy trials were presented for each of the 8 within-subjects conditions.

\section{Statistical analysis}

Reaction times and error rates were recorded for each participant and mean reaction time was calculated for each experimental condition. Following a well established statistical analysis procedure (Tucker and Ellis, 1998; Vainio et al., 2007), trials with 
reaction times deviating by $>2$ SDs from the participant's mean were excluded from the analysis [mean eliminated trials 23.6 (4.2\% of all 560 trials per subject)].

The orientation affordance effect has been defined as a shorter reaction time when the side of the response hand and the orientation of the presented prime object are identical (e.g., a response with the left hand to an object oriented to the left) compared with when they differ (e.g., a response with the left hand to an object oriented to the right) (Tucker and Ellis, 1998; Vainio et al., 2007). To facilitate the search for more specific interactions (e.g., group x object category interaction for the affordance effect), we computed an "affordance index" separately for each object category across both hands according to the following formula:

$$
\mathrm{AI}=\left[\frac{\left(L H_{R O}-L H_{L O}\right)}{\left(L H_{R O}+L H_{L O}\right)}+\frac{\left(R H_{L O}-R H_{R O}\right)}{\left(R H_{L O}+R H_{R O}\right)}\right] / 2
$$

where LHRO is the mean reaction time for the left hand and right-oriented objects; LHLO is the mean reaction time for the left hand and left-oriented objects; RHLO is the mean reaction time for the right hand and left-oriented objects; and RHRO is the mean reaction time for the right hand and right-oriented objects. Thus, a higher affordance index for an object category indicates shorter reaction times for congruent combination between responding hand and orientation of the respective object (i.e., left hand and left object orientation, right hand and right object orientation) compared with an incongruent combination of the same (i.e., left hand and right object orientation, right hand and left object orientation). A spatially compatible combination is considered to activate the associated motor representation [(Tucker and Ellis, 1998; Symes et al., 2007; Vainio et al., 2007; Riggio et al., 2008); see discussion for more information on the nature of the affordance effect)]. Therefore a higher affordance index would be an indicator for an activated motor representation and thus a facilitated response. We performed two repeated-measures ANOVAs: in the first one we searched for a general affordance effect and used the reaction times as dependent variable, hand, object orientation, and object category as within-subject factors, and group membership as between-subjects factor. In the second ANOVA we looked for differential affordance effects depending on the stimulus category and the group membership, i.e., we used the affordance index as dependent variable, object category as within-subject factor and group membership as between-subjects factor. The individual affordance indices of the smokers for everyday and smoking-related objects were correlated (Pearson correlation) with the corresponding FTND scores.

\section{Results}

\section{fMRI study}

The two-way whole-brain RFX ANOVA with the factors "stimulus category" (smoke vs. control) and "group" (smokers vs. nonsmokers) revealed a significant stimulus category $x$ group interaction in the regions listed in Table 1 ( $F \geq 6.1 ; p<$ 0.001 , corrected for multiple comparisons). The ROI-based t tests indicated higher 
fMRI activation for smokers compared with nonsmokers during the presentation of smoking cues in mainly two sets of brain regions which were identified by the whole-brain RFX ANOVA interaction. The first set consisted of left ventral and dorsal striatum, dorsolateral prefrontal cortex (DLPFC), insula (Figure 1B), uncus and medial frontal gyrus, right posterior subcallosal gyrus, and bilateral parahippocampal gyrus (PhG). In line with our hypotheses, the second brain network comprised bilateral premotor cortex (PMC) (Figure 1A), left superior parietal lobule (SPL) (Figure 1C), and right lateral cerebellum (Figure 1D).

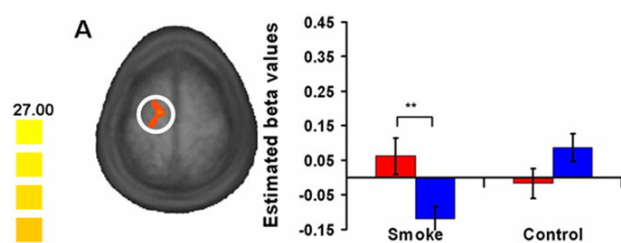

left premotor cortex, $z=61$

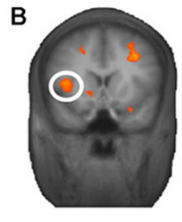

left insula, $y=12$

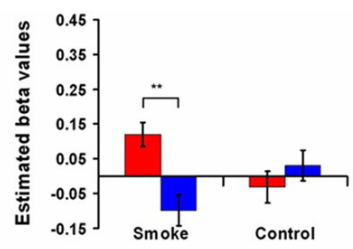

Control

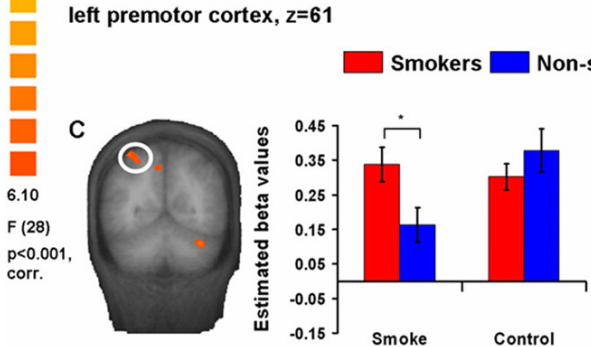

left superior parietal lobule, $y=-54$

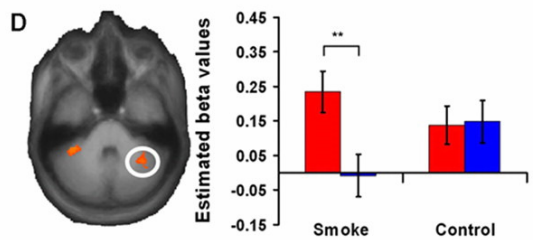

right cerebellum, $z=-32$

Figure 1. Neural cue reactivity in smokers and nonsmokers. A whole-brain RFX ANOVA interaction "stimulus category x group" revealed mainly two networks of brain regions, where ROI-based $t$ tests showed higher fMRI activations in smokers than nonsmokers while viewing smoking-related images. The first network consisted of regions related to craving and reward motivation (e.g., insula, see $\boldsymbol{B}$ ), and the second one comprised regions associated with skillful tool use and action knowledge (e.g., premotor cortex, superior parietal lobule, and lateral cerebellum; see $\boldsymbol{A}, \boldsymbol{C}$, and $\boldsymbol{D}$, respectively). The scale on the left side indicates minimum and maximum $F$ values, the degrees of freedom, and the significance level for the interaction. Error bars indicate SE from the mean; ${ }^{*} p<0.05 ; * * p<0.01$.

For the analysis of correlations between the individual degree of nicotine dependence and fMRI cue reactivity, a whole-brain RFX ANCOVA with the contrast "smoke $>$ control" and the covariate "FTND score" was computed $(n=15$ smokers) (Table 2). The $\beta$ weights of the GLM-contrast "smoke $>$ control" and the individual FTND scores were significantly correlated in left SPL $(r=0.67, p<0.01)$ and a region comprising parts of posterior middle temporal gyrus/inferior temporal cortex/fusiform gyrus ( $\mathrm{pMTG} / \mathrm{ITC} / \mathrm{FG} ; \mathrm{r}=0.70, \mathrm{p}<0.01$ ), right premotor cortex $(\mathrm{r}=0.66, \mathrm{p}<0.01), \mathrm{ITC} / \mathrm{FG}(\mathrm{r}=0.69, \mathrm{p}<0.01)$, middle frontal gyrus $(\mathrm{MFG} ; \mathrm{r}=0.68$, $\mathrm{p}<0.01)$, and middle occipital gyrus $(\mathrm{r}=0.67, \mathrm{p}<0.01)$ (Figure 2$)$.

\section{Behavioral study}

There were no main effects or interactions involving the factors object category or group on reaction times or error rates (Figure $3 \mathrm{~A}$ ). However, we found a hand $\mathrm{x}$ object orientation ANOVA interaction $(\mathrm{p}<0.05)$, i.e., a general affordance effect. 

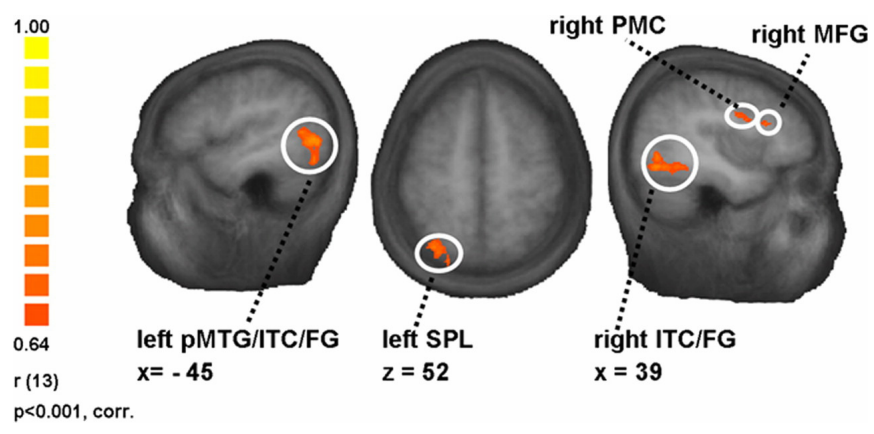

Figure 2. Association between neural cue reactivity in smokers and the individual degree of nicotine dependence. The figure illustrates some of the regions where the covariate "FTND score" correlated significantly with the $\beta$-values of the whole-brain RFX ANCOVA contrast "smoke $>$ control" $(n=15$ smokers). For a full list of the regions, see Table 2. The scale on the left side indicates minimum and maximum $r$ values, the degrees of freedom, and the significance level for the whole-brain RFX ANCOVA.

\section{Behavioral study}

There were no main effects or interactions involving the factors object category or group on reaction times or error rates (Figure 3A). However, we found a hand $\mathrm{x}$ object orientation ANOVA interaction $(\mathrm{p}<0.05)$, i.e., a general affordance effect. This interaction was attributable to shorter reaction times for compatibility compared with incompatibility between responding hand and object orientation of the priming visual object. There was a hand $\mathrm{x}$ object orientation $\mathrm{x}$ group $\mathrm{x}$ object category interaction $(\mathrm{p}<0.05)$ which indicated that the facilitation of responses compatible to object orientation depended on group membership and object category. To present this specific effect more clearly, we computed "affordance indices" indicating the amplitude of the respective affordance (i.e., facilitation) effect on the basis of the reaction times (see Materials and Methods). The ANOVA with the affordance index as dependent variable revealed a group $\mathrm{x}$ object category interaction $(\mathrm{p}<0.05)$ (Figure 3B). While smokers and nonsmokers showed comparable affordance effects for everyday nonsmoking objects, smoking paraphernalia led to affordance effects in smokers only. Furthermore, in smokers the FTND scores were significantly correlated with the affordance effect for smoking-related objects $(\mathrm{r}=0.71, \mathrm{p}=0.05)$ (Figure $3 \mathrm{C}$ ) but not for everyday objects $(\mathrm{r}=-0.4)$ (Figure 3D). The whole-brain RFX ANCOVA with the contrast "smoke $>$ control" and the covariate "affordance index for smoking objects" $(n=8$ smokers) (Table 3) revealed left PMC $(r=0.90, p<0.01)$, SPL $(r=0.90, p<0.01)$, MFG $(\mathrm{r}=0.90, \mathrm{p}<0.01)$, and Brodmann area 7 in the parietal lobe $(\mathrm{r}=0.90$, $\mathrm{p}<0.01)$ as well as right ITC $(\mathrm{r}=0.90, \mathrm{p}<0.01)$ and a region in cuneus/precuneus $(\mathrm{r}=0.90, \mathrm{p}<0.01)$ (Figure 4$)$. The behavioral orientation affordance experiment showed an increased behavioral responsiveness (in terms of the orientation affordance effect) to smoking-related objects in smokers than nonsmokers.

\section{ROI-based analyses}

Within the regions revealed by the whole-brain RFX ANCOVA with the contrast "smoke > control" and the covariate "FTND score", a further ROI-based RFX ANCOVA was computed with the contrast "smoke > control" and the covariate 
"affordance index for smoking objects" ( $\mathrm{n}=8$ smokers) (Table 4). Left SPL ( $\mathrm{r}=0.72$, $\mathrm{p}<0.05)$ and $\mathrm{pMTG} / \mathrm{ITC} / \mathrm{FG}(\mathrm{r}=0.74, \mathrm{p}<0.05)$ showed significant correlations not only for the whole-brain, but also for the ROI-based RFX ANCOVA. All of the regions revealed by the whole-brain RFX ANCOVA with the contrast "smoke>control" and the covariate "affordance index for smoking objects" with the exception of left BA 7 and right cuneus/precuneus showed significant correlations also in the ROI-based RFX ANCOVA $(n=8$ smokers, significant correlations varying between 0.76 and 0.80 ) with the contrast "smoke $>$ control" and the covariate "FTND" (Table 5).
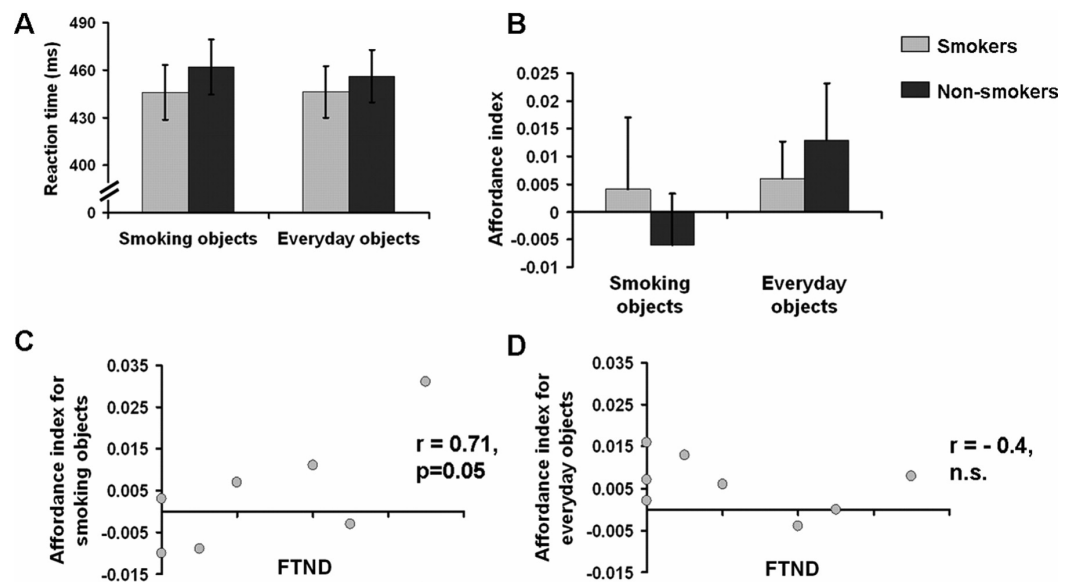

Figure 3. Behavioral cue reactivity of smokers and nonsmokers and association of the automatized responsiveness of smokers to the individual level of nicotine dependence. $\boldsymbol{A}$. Reaction times (means and SEs) of the two groups (smokers vs. nonsmokers) for the two object categories (smoking objects vs. everyday objects). B. Affordance indices (means and SEs) of the two groups (smokers vs. nonsmokers) for the two object categories (smoking objects vs. everyday objects) (ANOVA interaction, $p<0.05$ ). $\boldsymbol{C}$. Correlation between smokers' affordance indices for smoking objects and FTND scores $(r=0.71, p=0.05$; $n=8$; two of the smokers have identical affordance indices and FTND scores). $\boldsymbol{D}$. Correlation between smokers' affordance indices for everyday objects and FTND values $(r=-0.4$, n.s.; $n=8)$.

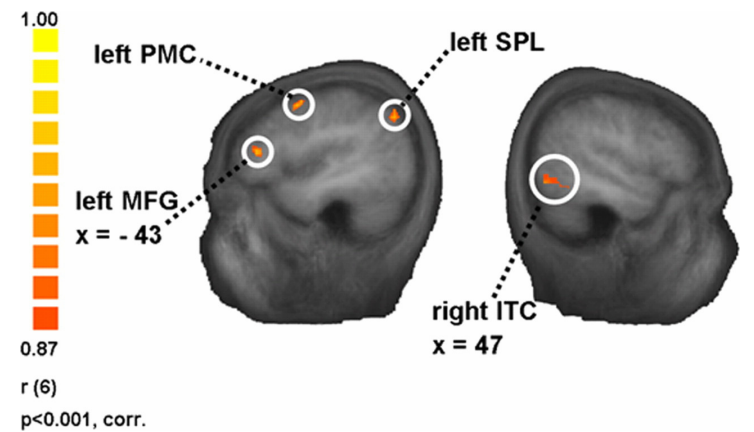

Figure 4. Association between neural cue reactivity in smokers and automatized behavioral responsiveness to smoking cues. The figure illustrates some of the regions where the covariate "affordance index for smoking objects" was significantly correlated with the $\beta$-values of the whole-brain RFX ANCOVA contrast "smoke $>$ control" ( $n=8$ smokers). For a full list of the regions, see Table 3. The scale on the left side indicates minimum and maximum $r$ values, the degrees of freedom, and the significance level for the whole-brain RFX ANCOVA. 


\section{Discussion}

Our analyses revealed mainly two networks of brain regions. The first one consisted of regions known to mediate the motivational aspects of addiction, while the second one consisted of brain regions related to tool use knowledge and action representation. Activations of some of the latter regions were correlated with the level of nicotine dependence.

The first network comprised the left ventral and dorsal striatum, DLPFC, insula, uncus, and medial frontal gyrus as well as right posterior subcallosal gyrus and bilateral $\mathrm{PhG}$. These regions have been associated with subjective responses of craving and the reward system (Breiter et al., 1997; Brody et al., 2002; Grusser et al., 2004; Wilson et al., 2004; Chiamulera, 2005; Naqvi et al., 2007; Fregni et al., 2008). The ventral striatum has been proposed to mediate the reinforcing effects of drugs (Drevets et al., 1999; Drevets et al., 2001; Kelley and Berridge, 2002; Volkow et al., 2004). The dorsal striatum is known for its role in stimulus-response learning and habitual behaviors (Packard and Knowlton, 2002) and may be of great importance for compulsive drug taking behavior (Gerdeman et al., 2003; Hyman et al., 2006). Moreover, dorsal striatal dopaminergic activity has been shown to be associated with cue-induced cocaine craving and could thus reflect the automatized nature of craving in later stages of addiction (Volkow et al., 2006). Ventral domains of the striatum may be more important for the early stages of addiction, whereas the more dorsal domains may take over control in the later stages, when drug usage is easily triggered by conditioned stimuli and is executed automatically (Everitt and Robbins, 2005). The higher activations in smokers compared with nonsmokers when exposed to smoking cues may thus reflect reward-related processes or experience of craving. Activations in the dorsal striatum may form the neural basis for the habitual link between conditioned cues and smoking behavior. The fact that smokers exhibited higher BOLD signals in both the ventral and dorsal parts of the striatum can be attributed to the fact that the smokers' group consisted both of light and heavy smokers. Interestingly, we found no correlations between the degree of nicotine dependence and activations in this craving-related network.

The second network comprising bilateral PMC, left SPL, and right lateral cerebellum might underlie the representation of drug taking skills as well as the preparation and efficient execution of the specific action sequences. These regions are thought to form part of a system representing tool use skills and action knowledge (Grafton et al., 1997; Chao and Martin, 2000; Grezes et al., 2003; Johnson-Frey, 2004) and to be crucial for the efficient execution of the corresponding actions (Creem-Regehr and Lee, 2005; Lewis, 2006). The premotor cortex is important for linking intent or knowledge of how to execute an action to the following sequence of motor commands as well as for selecting actions on the basis of arbitrary cues (Fink et al., 1999; Arbib et al., 2000; Grezes et al., 2003). The lateral cerebellum is known to encode skilled action representations, internal models of tools, and automatized actions (Imamizu et al., 2003; Higuchi et al., 2007). The left superior parietal lobule has a prominent role in planning and executing tool use movements (Johnson-Frey et al., 2005). Apparently, smoking cues automatically activate smokers' neural representations of the corresponding tool use skills and action knowledge. 
The association between the individual level of nicotine dependence and the $\beta$-values from the whole-brain RFX ANCOVA in left SPL, left pMTG/ITC/FG, and right PMC and MFG further supports the assumed relationship between the neural representations of drug-related action schemata which are dependent on the smoker's learning history (Tiffany, 1990), and the severity of nicotine dependence. The FG/ITC is related both to the analysis of object features and to the interpretation of visual action (Haxby et al., 2001; Chaminade et al., 2005). The pMTG is involved in processing action knowledge (Johnson-Frey et al., 2005). Several studies have pointed out the importance of MFG for skilled movement and tool-associated action identification (Grabowski et al., 1998; Haaland et al., 2000). Increased smoking expertise may thus include an enhanced ability to activate the respective smoking skill and action representations. Behavioral evidence also suggests that habitual, automatized actions are a frequent reason for smoking in more dependent smokers (Piasecki et al., 2007).

The absence of a correlation between FTND score and fMRI activation in craving-related brain regions may be attributable to the fact that our participants were not nicotine-deprived. Indeed, there is evidence that nicotine deprivation and cigarette availability affect cue reactivity (Droungas et al., 1995; Carter and Tiffany, 2001; McBride et al., 2006). However, our aim was to investigate reactivity as closely as possible to natural everyday smoking situations. Furthermore, it has been shown that craving occurs also in natural environments without an experimentally induced nicotine deprivation (Piasecki et al., 2007).

Our second experiment investigated the orientation affordance effect as a function of group and object category. Orientation affordance refers to faster responses for spatial compatibility between the responding hand and the orientation of the priming image of a graspable object. It has been proposed that this facilitation of reactions is attributable to the automatic activation of corresponding motor representations by the priming object(Tucker and Ellis, 1998; Symes et al., 2007; Vainio et al., 2007; Riggio et al., 2008). Thus, the affordance effect represents a suitable measure of automatized smoking-related action representations. Our data provide evidence for a general affordance effect, indicating faster reactions for congruency between the responding hand and the orientation of the priming object. Smokers and nonsmokers exhibited comparable affordance effects for common nonsmoking objects. Smoking paraphernalia led to affordance effects in smokers only, i.e., reactions to smoking-related objects were facilitated by compatible object orientation in smokers but not in nonsmokers. Thus, smokers' responses to smoking paraphernalia can be triggered as automatically and easily as to comparable everyday objects. Furthermore, experience-based motor representations of smoking actions have been established in smokers only.

Although there was a general affordance effect and a "group x object category" interaction for the affordance index, there were no general reaction time differences between the groups and no "group x object category" interaction for the reaction times. This was not unexpected since only the affordance index takes the compatibility between responding hand and object orientation into account and measures the action facilitation effect.

The FTND scores correlated with the affordance effect of smokers for smoking but not for everyday objects. Furthermore, the whole-brain RFX ANCOVA revealed remarkable correlations between the covariate "affordance index for 
smoking objects" and fMRI cue reactivity in left PMC, SPL, MFG, and right ITC. This shows that automatized behavioral responsiveness to smoking-associated cues is more pronounced in heavier smokers and is related to the neural underpinnings of drug taking skills. Thus, the smokers' experience with smoking-related objects was reflected in both behavioral affordance profiles and the activation of tool use-related brain regions.

The fMRI cue reactivity in the majority of those regions which were identified by the whole-brain RFX ANCOVAs using the one covariate also showed a significant ROI-based correlation with the other covariate. This further supports the association of fMRI activation of action-related brain regions with the severity of nicotine dependence and automatized behavioral responsiveness to smoking-related objects.

The strong activation of smoking-related action schemata in smokers might either be interpreted as a cause of smoking or as an effect of exaggerated consumption. Research on experience-based neural plasticity shows that intensive engagement in an activity leads to stronger neural representations of the respective activity and the used objects (Pearce et al., 2000; Sanes and Donoghue, 2000; CalvoMerino et al., 2006; Schwenkreis et al., 2007; Weisberg et al., 2007). Therefore, we suggest that strong activation of the smoking-related action schemata is the consequence of the frequently executed process of smoking. On the other hand, triggered schemata may also reduce the threshold for executing the action of smoking, thus contributing to the high smoking relapse rates.

Together, our findings confirm that nicotine dependence involves striatum, insula, and dorsolateral prefrontal cortex, which have been intensively studied in relation to craving and drug seeking habits. The present results provide new evidence that smoking-associated images can activate neural representations in brain regions probably encoding the respective drug taking skills and action knowledge. Responses of action-related regions to smoking cues may thus represent a novel neural correlate of nicotine dependence. On the behavioral level, smoking paraphernalia activate motor representations in smokers but not in nonsmokers as easily and automatically as similarly familiar objects. This effect is linked to the fMRI cue reactivity of smokers in tool use and action-related brain regions. Both neural and behavioral correlates of the drug taking skills and action knowledge correlate with the level of nicotine dependence. The activation of such deeply embedded action schemata might contribute to the high relapse risk in nicotine addiction. We hope that these findings will initiate the development of novel neural and behavioral markers of therapy success or risk of relapse, and guide new therapeutic strategies addressing the importance of involuntary, automatic action sequences. 


\section{Tables}

Table 1. Brain regions showing significant whole brain ANOVA interaction "stimulus category x group" $(\mathrm{df}=28, \mathrm{p}<0.001$, corrected for multiple comparisons $)$ and ROI-based unpaired, two-tailed t-tests $(\mathrm{df}=28)$ for group differences between responses to stimuli from the "smoke" vs. "control" categories. a = Positive t-values indicate higher beta values for the smokers compared to non-smokers. Negative t-values indicate higher beta values for non-smokers compared to smokers.

\begin{tabular}{|c|c|c|c|c|c|c|c|c|c|}
\hline \multirow[t]{2}{*}{ Region } & \multicolumn{3}{|c|}{$\begin{array}{c}\text { Talairach } \\
\text { coordinates }\end{array}$} & \multirow{2}{*}{$\begin{array}{l}\mathrm{Nr} \text { of } \\
\text { voxels }\end{array}$} & \multirow[t]{2}{*}{ F-value } & \multicolumn{2}{|c|}{ Smoke stimulia } & \multicolumn{2}{|c|}{ Control stimuli ${ }^{\mathrm{a}}$} \\
\hline & $\mathrm{x}$ & $\mathrm{y}$ & $\mathrm{z}$ & & & t- & $\mathrm{p}$ & t-value & $\mathrm{p}$ \\
\hline $\begin{array}{l}\text { left ventral/dorsal } \\
\text { striatum }\end{array}$ & -11 & 10 & -2 & 3069 & 8.23 & 4.838 & $0.000 * *$ & -1.133 & n. s. \\
\hline left insula & -36 & 13 & 9 & 1485 & 7.84 & 3.856 & $0.001 * *$ & -0.975 & n. s. \\
\hline $\begin{array}{l}\text { left dorsolateral } \\
\text { prefrontal cortex }\end{array}$ & -39 & 30 & 31 & 2690 & 10.14 & 3.336 & $0.002 * *$ & -1.709 & n. s. \\
\hline $\begin{array}{l}\text { left medial frontal } \\
\text { gyrus }\end{array}$ & -19 & 9 & 43 & 514 & 6.76 & 2.925 & $0.007 * *$ & -1.906 & n. s. \\
\hline left premotor cortex & -15 & -12 & 59 & 1066 & 7.84 & 2.879 & $0.008 * *$ & -1.781 & n. s. \\
\hline right cerebellum & 33 & -48 & -28 & 1259 & 7.64 & 2.837 & $0.008 * *$ & -0.123 & n. s. \\
\hline left uncus & -31 & 2 & -19 & 706 & 8.61 & 2.766 & $0.01 *$ & -2.842 & $0.008 * *$ \\
\hline $\begin{array}{l}\text { left parahippocampal } \\
\text { gyrus }\end{array}$ & -13 & -28 & -7 & 973 & 7.68 & 2.742 & $0.011 *$ & -2.461 & $0.02 *$ \\
\hline $\begin{array}{l}\text { right subcallosal } \\
\text { gyrus, Brodmann } \\
\text { area } 34\end{array}$ & 28 & 4 & -12 & 1866 & 8.53 & 2.522 & $0.018 *$ & -1.724 & n. s. \\
\hline $\begin{array}{l}\text { left superior parietal } \\
\text { lobule }\end{array}$ & -24 & -61 & 46 & 2050 & 8.28 & 2.496 & $0.019 *$ & -1.029 & n. s. \\
\hline $\begin{array}{l}\text { right } \\
\text { parahippocampal } \\
\text { gyrus }\end{array}$ & 32 & -28 & -20 & 1594 & 7.67 & 2.468 & $0.02 *$ & -1.915 & n. s. \\
\hline right premotor cortex & 31 & 12 & 38 & 2117 & 7.58 & 2.123 & $0.043 *$ & -2.146 & $0.041 *$ \\
\hline left cerebellum & -35 & -39 & -30 & 435 & 7.83 & 1.700 & n. s. & -2.243 & $0.035 *$ \\
\hline $\begin{array}{l}\text { right posterior middle } \\
\text { temporal gyrus }\end{array}$ & 50 & -61 & 6 & 1057 & 8.22 & 1.377 & n. s. & -1.069 & n. s. \\
\hline $\begin{array}{l}\text { left posterior middle } \\
\text { temporal gyrus }\end{array}$ & -38 & -75 & 11 & 656 & 7.19 & 1.007 & n. s. & -1.842 & n. s. \\
\hline $\begin{array}{l}\text { right posterior } \\
\text { cingulate gyrus }\end{array}$ & 24 & -64 & 8 & 879 & 8.64 & 0.824 & n. s. & -3.484 & $0.002 * *$ \\
\hline $\begin{array}{l}\text { right middle temporal } \\
\text { gyrus/occipital lobe }\end{array}$ & 42 & -70 & 14 & 791 & 7.39 & 0.242 & n. s. & -2.052 & $0.05 *$ \\
\hline
\end{tabular}


Table 2. Brain regions showing significant results for the whole-brain RFX ANCOVA with contrast "smoke $>$ control" and covariate FTND $(\mathrm{df}=13, \mathrm{p}<0.001$, corrected for multiple comparisons $)$

\begin{tabular}{lcccccc}
\hline \multirow{2}{*}{ Region } & \multicolumn{2}{c}{ Talairach coordinates } & & \\
\cline { 2 - 4 } & $\mathrm{x}$ & $\mathrm{y}$ & $\mathrm{z}$ & & \\
\cline { 2 - 5 } & -40 & -66 & -2 & 4021 & 0.70 \\
$\begin{array}{l}\text { left posterior middle temporal gyrus/inferior } \\
\text { temporal cortex/fusiform gyrus }\end{array}$ & 36 & -63 & -8 & 4405 & r-value \\
$\begin{array}{l}\text { right inferior temporal cortex/ } \\
\text { fusiform gyrus }\end{array}$ & 37 & 3 & 28 & 418 & 0.69 \\
$\begin{array}{l}\text { right premotor cortex } \\
\text { right middle frontal gyrus }\end{array}$ & 36 & 22 & 22 & 379 & 0.66 \\
right middle occipital gyrus & 30 & -79 & 8 & 555 & 0.67 \\
left superior parietal lobule & -27 & -66 & 52 & 834 & 0.67 \\
\hline
\end{tabular}

Table 3. Brain regions showing significant results for the whole-brain RFX ANCOVA with contrast "smoke > control" and covariate "affordance index for smoking objects" $(\mathrm{df}=6, \mathrm{p}<0.001$, corrected for multiple comparisons)

\begin{tabular}{lccccc}
\hline \multirow{2}{*}{ Region } & \multicolumn{2}{c}{ Talairach coordinates } & & \\
\cline { 2 - 4 } & $\mathrm{x}$ & $\mathrm{y}$ & $\mathrm{z}$ & & \\
\hline left premotor cortex & -34 & 2 & 51 & 1653 & 0.90 \\
left superior parietal lobule & -37 & -66 & 41 & 944 & 0.90 \\
right inferior temporal cortex & 44 & -59 & -5 & 649 & 0.90 \\
left middle frontal gyrus & -45 & 35 & 14 & 588 & 0.90 \\
left parietal lobe, BA 7 & -10 & -51 & 36 & 529 & 0.90 \\
right cuneus/precuneus & -28 & -70 & 15 & 527 & 0.90 \\
\hline
\end{tabular}


Chapter 3

Table 4. ROI-based RFX ANCOVA with the contrast "smoke $>$ control" and the covariate "affordance index for smoking objects" $(\mathrm{df}=6)$ was computed within brain regions showing significant results for the whole-brain RFX ANCOVA with contrast "smoke > control" and covariate FTND.

\begin{tabular}{|c|c|c|c|c|c|}
\hline \multirow{2}{*}{ Region } & \multicolumn{3}{|c|}{ Talairach coordinates } & \multirow{2}{*}{$\mathrm{Nr}$ of voxels } & \multirow{2}{*}{$\begin{array}{c}\text { r-value } \\
\text { (ROI-based ANCOVA) }\end{array}$} \\
\hline & $\mathrm{x}$ & $\mathrm{y}$ & $\mathrm{z}$ & & \\
\hline $\begin{array}{l}\text { left posterior middle temporal } \\
\text { gyrus/inferior temporal } \\
\text { cortex/fusiform gyrus }\end{array}$ & -40 & -66 & -2 & 4021 & 0.74 \\
\hline left superior parietal lobule & -27 & -66 & 52 & 834 & 0.72 \\
\hline right premotor cortex & 37 & 3 & 28 & 418 & n. s. \\
\hline right middle occipital gyrus & 30 & -79 & 8 & 555 & n. s. \\
\hline $\begin{array}{l}\text { right inferior temporal cortex/ } \\
\text { fusiform gyrus }\end{array}$ & 36 & -63 & -8 & 4405 & n.s. \\
\hline right middle frontal gyrus & 36 & 22 & 22 & 379 & n. s. \\
\hline
\end{tabular}

Table 5. ROI-based RFX ANCOVA with the contrast "smoke > control" and the covariate "FTND" $(\mathrm{df}=6)$ was computed within brain regions showing significant results for the whole-brain RFX ANCOVA with contrast "smoke > control" and covariate "affordance index for smoking objects".

\begin{tabular}{lccccc}
\hline \multirow{2}{*}{ Region } & \multicolumn{3}{c}{ Talairach coordinates } & & \multicolumn{1}{c}{$\begin{array}{c}\text { r-value } \\
\text { (ROI-based ANCOVA) }\end{array}$} \\
\cline { 2 - 4 } & $\mathrm{x}$ & $\mathrm{y}$ & $\mathrm{z}$ & & 0.80 \\
\hline left superior parietal lobule & -37 & -66 & 41 & 944 & 0.79 \\
$\begin{array}{l}\text { left premotor cortex } \\
\text { right inferior temporal }\end{array}$ & -34 & 2 & 51 & 1653 & 0.77 \\
$\begin{array}{l}\text { cortex } \\
\text { left middle frontal gyrus }\end{array}$ & -44 & -59 & -5 & 649 & 0.76 \\
right cuneus/precuneus & -28 & -70 & 15 & 527 & n. s. \\
left parietal lobe, BA 7 & -10 & -51 & 36 & 529 & n. s. \\
\hline
\end{tabular}




\section{Chapter 4}

\section{Sensory modality of smoking cues modulates neural cue reactivity}

\section{Based on:}

Yalachkov Y, Kaiser J, Goerres A, Seehaus A, Naumer MJ. Sensory modality of smoking cues modulates neural cue reactivity. (in preparation for submission to Archives of General Psychiatry) 


\begin{abstract}
Attempts to identify brain regions activated by addiction-related cues have yielded inconsistent findings. The present study on nicotine addiction tested whether neural responses to drug cues are modulated by the sensory modality of the presented stimuli. We measured brain activation with functional magnetic resonance imaging (fMRI) in smokers and non-smokers while they either viewed images of smoking paraphernalia and control objects or touched the same objects without seeing them. Smoking-related stimuli presented in the haptic modality induced more pronounced neural cue reactivity than visual cues in the left dorsal striatum of smokers compared to non-smokers. Similarly, within-subject analyses in smokers revealed stronger neural cue reactivity for haptic stimuli in the left dorsal striatum as well as in the right premotor cortex and dorsolateral prefrontal cortex. Conversely, an increased fMRI cue reactivity for visual compared to haptic smoking cues was found in the right cerebellum of smokers. Apparently, cue reactivity of brain regions involved in crucial addiction-related processes such as habits and automatized action schemata as well as reward motivation and decision-making is modulated by the sensory modality in which the smoking cues are presented.
\end{abstract}




\section{Introduction}

During the last decades addiction research has focused on elucidating the neurobiological mechanisms of reward, learning, and memory, which are hijacked by addictive drugs and contribute to the development of pathological incentive values of drugs and their associated cues (Hyman et al., 2006). The emergence of drug-seeking habits and automatized action schemata controlled by conditioned stimuli has received particular attention (Tiffany, 1990; Everitt and Robbins, 2005; Belin and Everitt, 2008; Yalachkov et al., 2009). Recently, there has also been a growing interest in addiction-related dysfunctions of neural processes which reflect decision-making (Garavan and Hester, 2007; Goldstein et al., 2009). These functions have mostly been studied in experiments using cue reactivity paradigms during which addicts are exposed to drug-related stimuli while their behavioral and/or physiological responses are recorded. Since drug consumption is triggered easily by drug-associated stimuli, studying the mechanisms of cue reactivity in the laboratory promises important insights into the processes underlying cue-induced drug-seeking and relapse in everyday life (Tiffany, 1990; Carter and Tiffany, 1999).

A growing body of evidence has shown that the addicted human brain exhibits differential patterns of activation when confronted with drug-related as compared to neutral cues (Volkow et al., 2004; Fowler et al., 2007). However, neuroimaging results have been rather inconsistent, showing that the neurocircuits of addiction are not activated to the same extent in different studies and indicating that different factors such as for example the perceived availability of the drug may modulate the neural and behavioral responses to drug cues (Wilson et al., 2004).

Interestingly, the use of different sensory modalities for the presentation of drug cues has rarely been considered as a possible source of these inconsistencies, although behavioral experiments have demonstrated pronounced differences in the ability of drug cues to elicit behavioral and psychophysiological reactions depending on the sensory modality of presentation (Johnson et al., 1998; Reid et al., 2006). The ability to detect brain regions underlying neural cue reactivity in addiction might similarly be linked to the sensory modality in which the drug cues occur. This would implicate important consequences not only for addiction-related experimental designs but also for the development of exposure therapies and biomarkers of addiction.

In the present experiment, we employed functional magnetic resonance imaging (fMRI) to address these issues in the context of smoking. Subjects touched smoking paraphernalia and control objects without seeing them or viewed their greyscaled images while executing a movement similar to the haptic exploration but without receiving any tactile stimulation. We examined the influence of the object category (smoking vs. control) and modality (visual vs. haptic) as well as the participants' smoking status (smokers vs. non-smokers) on neural cue reactivity. Additionally, we tested the smokers and non-smokers separately for within-subject effects and explored whether the effects in smokers are linked to the individual degree of nicotine addiction. 


\section{Materials and Methods}

\section{Subjects}

All participants were right-handed, had normal or corrected-to-normal vision and were students or university academic staff. The smokers $(n=15$, mean age 28.3 years $[\mathrm{SD}=3.7], 8$ women) were included in the study only if they smoked at least one cigarette per day for at least two years. They were instructed to smoke according to their usual habits but to abstain from smoking 30 minutes before the fMRI recordings to reduce the confounding influence of nicotine on the blood oxygen level-dependent (BOLD) signal. Their individual level of nicotine dependence was assessed with the Fagerström Test for Nicotine Dependence [FTND; (Bleich et al., 2002)] after the fMRI sessions (mean FTND score $=2.8, \mathrm{SD}=2.4$ ). We recruited both light and heavy smokers to ensure that there was enough variation in the severity of nicotine addiction, facilitating the detection of possible correlations between test scores and neural cue reactivity. The non-smokers $(n=15$, mean age 27 years $[\mathrm{SD}=5.01], 9$ women) claimed that they had either never smoked or had smoked only a very limited number of cigarettes during their lifetime. Written informed consent was obtained after giving the subjects a complete description of the study. All subjects reported that they were free from neurological/psychiatric disorders and not taking any psychotropic medications at the time of the study.

\section{Experimental design}

Stimuli were four common everyday objects (a pen, a crème box, a small video cassette, an audio cassette; control objects condition) and four smoking-related objects (a cigarette, an ashtray, a normal cigarette box, a longer cigarette box; smoking paraphernalia condition) and gray-scaled pictures of these objects. The objects in the two categories were matched for size and weight and, as far as possible, for roughness of their surface.

During the fMRI recordings, participants actively touched the objects or viewed the images. Using a block design, the "smoking paraphernalia" and "control objects" conditions were presented either in the visual or haptic modality. Closing and reopening of the participant's grasping hand was cued by color changes of a centrally presented fixation cross which was present in all conditions. The participants had to follow the color change instructions irrespective of the experimental condition (i.e., also in the visual condition without haptic input). Thus, we created comparable motor components for all conditions. Haptic stimuli were presented to the participant's hand by an experimenter who received instructions about the on- and offset of stimulation via headphones. Additionally, there was a motor control condition during which participants merely closed and reopened their hand following the color changes of the fixation cross without receiving any further sensory stimulation. This resulted in 5 experimental conditions: visual control (VC), haptic control (HC), visual smoke (VS), haptic smoke (HS), and motor (M). Two further conditions, which were also included in the design, are not the subject of the present paper and will therefore not be reported.

The participants underwent four functional runs (two with the left and two with the right hand; the assignment of left or right hand to the respective run was 
pseudo-randomized) and one anatomical scan. At the beginning of each run a fixation interval and a dummy block ensured that T1 saturation effects were avoided. Each experimental condition was repeated twice in separate blocks within each run, resulting in $7 \times 2=14$ experimental blocks per functional run. The order of the blocks and the runs was pseudo-randomized. Blocks lasted for 24 seconds and alternated with fixation intervals of equal length. Thus, each of the four runs consisted of 15 blocks ( 14 experimental +1 dummy) and 16 fixation intervals, in total $31 \times 24=744 \mathrm{~s}$ or $12 \mathrm{~min}$. The entire experiment lasted approximately 55 minutes (4 functional runs x $12 \mathrm{~min}+1$ anatomical scan x $6.6 \mathrm{~min}$ ).

During each block, the following procedure was repeated four times: 3200 $\mathrm{ms}$ of fixation preceded a color change of the fixation cross lasting for $300 \mathrm{~ms}$ which instructed the participants to close their hand. The experimental stimulus was then presented for $2000 \mathrm{~ms}$ and another color change of the fixation cross signaled to the participants to reopen their hand. This was followed by another $500 \mathrm{~ms}$ of fixation. In the motor condition (M) no stimulus was presented during the $2000 \mathrm{~ms}$ and the participants viewed the default fixation cross. Thus, four stimuli from the same object category were presented in a randomized order within each block (4 x 6000 $\mathrm{ms}=24 \mathrm{~s}$ per block).

The stimuli were presented with Presentation software (Neurobehavioral Systems, Albany, CA, U.S.A.) running on a PC (Miditower Celeron) at a frame rate of $60 \mathrm{~Hz}$. Images were projected onto a vertical screen positioned inside the scanner with an LCD projector (Sony, VPL PX 20) equipped with a custom-made lens. Participants viewed the screen through a mirror fixed on the head coil. The participants' field of view was 52.5 degrees visual angle (maximum horizontal distance).

The fMRI procedure was performed on a 3-Tesla Magnetom Allegra scanner (Siemens, Erlangen, Germany) at the Brain Imaging Center in Frankfurt am Main. A gradient-recalled echo-planar imaging sequence with the following parameters was used: 33 slices; repetition time (TR) $2000 \mathrm{~ms}$; echo time (TE) 30 ms; field of view $192 \mathrm{~mm}$; in-plane resolution $3 \mathrm{~mm}^{2}$; slice thickness $3 \mathrm{~mm}$; gap thickness $0.3 \mathrm{~mm} ; 372$ volumes per functional run. For the anatomical imaging a magnetization-prepared rapid-acquisition gradient echo sequence (176 slices per slab, TR $2250 \mathrm{~ms}$, TE $2.6 \mathrm{~ms}$, flip angle $9 \mathrm{deg}$, matrix 256x256 and voxel size $1 \mathrm{~mm}^{3}$ ) was used.

\section{Statistical analysis}

Data were analyzed using the BrainVoyager QX 1.10.4 software package (Brain Innovation, Maastricht, The Netherlands). Preprocessing included the following steps: three-dimensional motion correction, linear-trend removal and temporal highpass filtering at $0.0054 \mathrm{~Hz}$, and slice-scan-time correction with sinc interpolation. Functional and Talairach-standardized anatomical data were used to build a voxel time course file and were spatially smoothed with a Gaussian filter of $8 \mathrm{~mm}$ full width at half maximum. For each voxel the time course was regressed on a set of dummy-coded predictors representing the experimental conditions. To account for the shape and delay of the hemodynamic response, the predictor time courses (boxcar functions) were convolved with a gamma function. 
First, we conducted a between-subjects analysis creating a whole-brain random-effects general linear model (RFX-GLM) using the data of all participants $(\mathrm{n}=30)$. Then an analysis of variance (ANOVA) with the within-subject factors "Object category" (control objects vs. smoking paraphernalia) and "Modality" (visual vs. haptic) and the between-subjects factor "Group" (non-smokers vs. smokers) was computed.

Next, using two group-specific whole-brain RFX-GLMs (n1 = 15 smokers; $\mathrm{n} 2=15$ non-smokers), we computed two additional whole-brain ANOVAs separately for smokers and non-smokers to search for specific within-subject cue reactivity effects. For each group-specific ANOVA two within-subject factors were defined: "Object category" (control objects vs. smoking paraphernalia) and "Modality" (visual vs. haptic).

Separate statistical maps were created for all main effects and interactions of the three ANOVAs. Where necessary, t-tests based on the beta-weights extracted from the respective brain region were performed to help interpreting the statistical interactions.

Finally, based on the smokers' data, we tested for correlations between the beta weights from statistical cue reactivity contrasts and the severity of nicotine dependence indicated by FTND scores. For this purpose a whole-brain analysis of covariance (ANCOVA, $\mathrm{n}=15$ smokers) with the external covariate "FTND score" and the statistical contrasts "VS > VC" and "HS > HC" was computed.

All statistical maps were corrected for multiple comparisons using the cluster-size thresholding method (Forman et al., 1995; Goebel et al., 2006) implemented in the "Cluster-level Statistical Threshold Estimator" plugin of BrainVoyager. In this method, for each statistical map the uncorrected voxel level threshold was set at $\mathrm{p}<0.01$ (uncorrected) and was then submitted to a whole-brain correction criterion based on the estimate of the spatial smoothness of the map and on an iterative procedure (Monte Carlo simulation) for estimating cluster-level falsepositive rates. After 1,000 iterations, the minimum cluster-size that yielded a clusterlevel false-positive rate of $5 \%$ was used to threshold the statistical map, thus resulting in a final $\mathrm{p}<0.05$, corrected for multiple comparisons.

The statistical maps for the main effect of the factor "Modality" in the whole-brain ANOVAs resulted in several clusters which comprised many thousand voxels. To obtain a clearer statistical map, we increased in all three whole-brain ANOVAs the statistical threshold for this factor to $\mathrm{p}<0.001$ (corrected for multiple comparisons using the False Discovery Rate [FDR]).

\section{Results}

\section{Between-subjects analysis}

The whole-brain ANOVA computed on the basis of all 30 subjects resulted in 7 statistical maps which reflected the main effects "Object category", "Modality", and "Group" as well as the interactions "Object category x Group", "Object category x Modality", "Group x Modality" and "Object category x Group x Modality" (Tables 1-14). 
The fMRI signal was higher in smokers in comparison to non-smokers across all of the experimental conditions (main effect "Group") in the right inferior frontal gyrus (IFG) and the supplementary motor area. Conversely, a higher fMRI activation across all conditions for non-smokers was found in occipital and temporal regions such as the right lingual gyrus, middle occipital gyrus, left fusiform gyrus, cuneus and middle temporal gyrus (Table 1). The interaction "Group x Object category" revealed significant effects in the bilateral lingual gyrus and the right middle temporal gyrus. In these regions the smokers showed a lower BOLD signal as compared to the non-smokers and this was more pronounced for the smoking paraphernalia than for the control objects (Table 2). An interaction between the three factors "Group", "Object category" and "Modality" was demonstrated only in the left dorsal striatum (nucleus caudatus and putamen), where the fMRI response to haptically presented smoking paraphernalia in comparison to control objects (HS HC) was higher for smokers than non-smokers. On the other hand, in the same brain region the visual cue reactivity $(\mathbf{V S}-\mathbf{V C})$ was lower for smokers than non-smokers (Figure $1 \&$ Table 3 ).
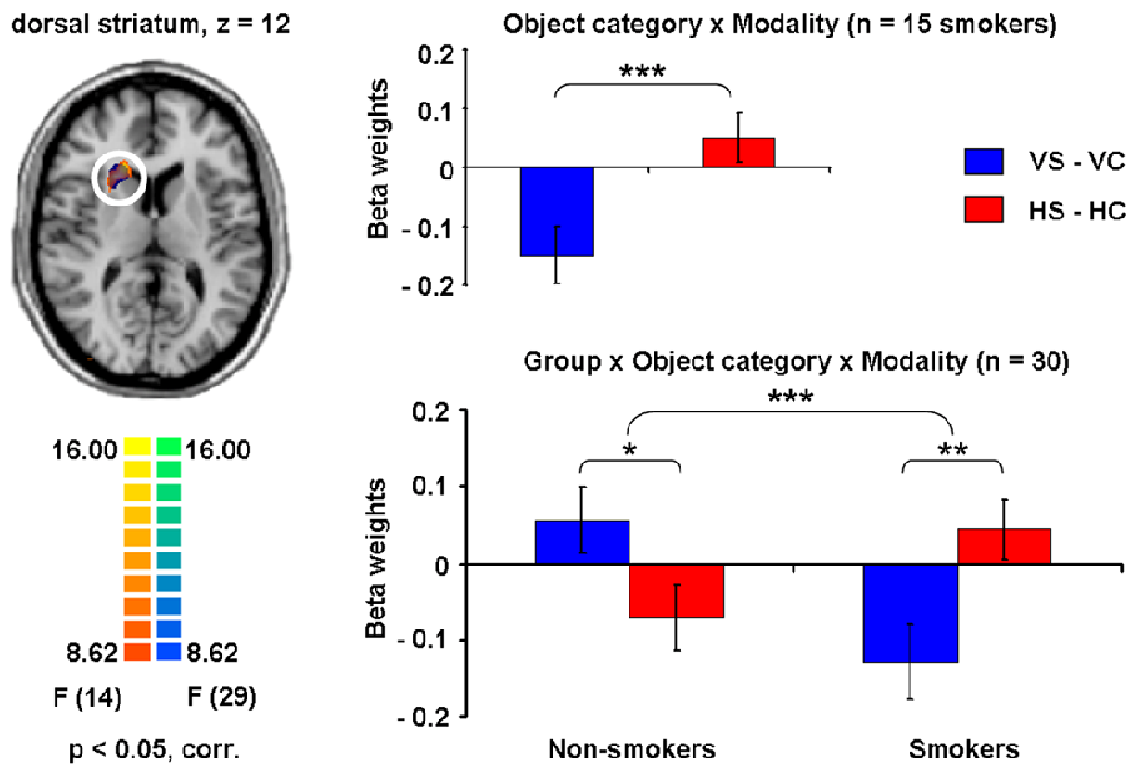

Figure 1. Neural cue reactivity of the dorsal striatum in smokers and nonsmokers. The interactions "Object category x Modality x Group" (whole-brain between-subjects ANOVA, $\mathrm{n}=30$; significant clusters in blue) and "Object category x Modality (whole-brain within-subjects ANOVA, $\mathrm{n}=15$ smokers; significant clusters in orange) revealed that the neural cue reactivity in the left dorsal striatum was modulated by the sensory modality of the presented cues. To illustrate the fMRI signal during the different conditions, beta weights for the respective control condition (C) were subtracted from the respective smoking condition (S) on a region-of-interest basis. The modality-specific beta weight differences were then plotted. The first plot on the right depicts the beta weights differences in the left dorsal striatum as defined by the within-subjects ANOVA interaction for the 15 smokers. The second one demonstrates the beta weights differences in the left dorsal striatum as defined by the between-subjects ANOVA interaction for all 30 subjects. Post-hoc t-tests were computed in each of the revealed brain regions to examine the interactions $\left(* \mathrm{p}<0.05 ;{ }^{* *} \mathrm{p}<0.01 ; * * \mathrm{p} \leq 0.001\right.$, for more information on the ttests see Tables $3 \& 5$ ). 


\section{Within-subjects analysis}

To further investigate neural cue reactivity in smokers, an additional whole-brain ANOVA was computed for the smokers' data only. A main effect of the factor "Object category" with a higher fMRI signal for control objects compared to smoking paraphernalia was found in the bilateral lingual gyrus and cuneus as well as in the right precentral gyrus (Table 4). Interestingly, several brain regions showed a differential activation pattern depending on object category and sensory modality ("Object category x Modality" interaction). In the right cerebellum, anterior prefrontal cortex (white matter, vicinity of Brodmann area 10) and medial temporal lobe (white matter, vicinity of the parahippocampal gyrus) as well as in the left middle occipital gyrus the fMRI activation to visual smoking cues as compared to visual control cues (VS - VC) was higher than to haptically presented stimuli (HS HC) (Figure $2 \&$ Table 5). The opposite activation pattern - higher neural cue reactivity for the haptic than for the visual modality - was observed in the right dorsolateral prefrontal cortex (DLPFC) and dorsal premotor cortex (PMd), in the left dorsal striatum (nucleus caudatus and putamen) and in a cluster lying in white matter
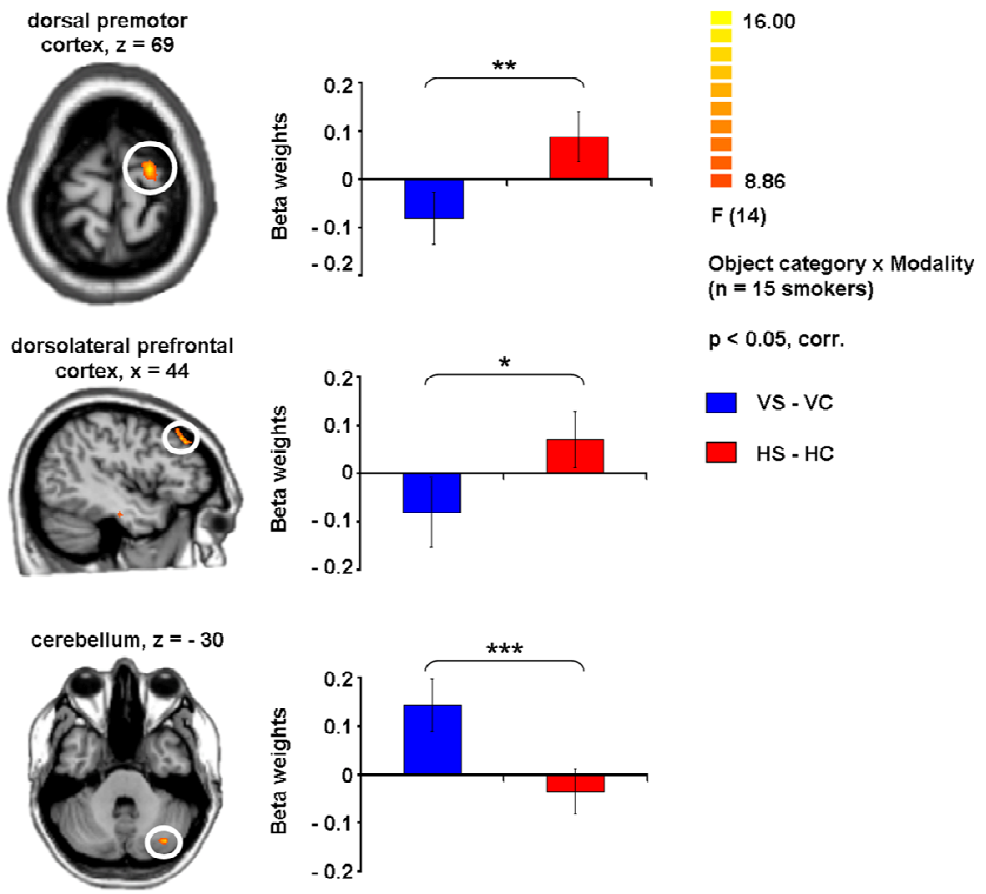

Figure 2. Neural cue reactivity of the dorsal premotor cortex, dorsolateral prefrontal cortex and cerebellum in smokers. The interaction "Object category $\mathrm{x}$ Modality (whole-brain within-subjects ANOVA, $\mathrm{n}=15$ smokers; significant clusters in orange) revealed that the neural cue reactivity in the right dorsal premotor cortex, dorsolateral prefrontal cortex and cerebellum was modulated by the sensory modality of the presented cues. To illustrate the fMRI signal during the different conditions, graphs on the right depict beta weights as a function of modality for the respective control condition (C) subtracted from the respective smoking condition $(\mathrm{S})$ on a region-of-interest basis. Post-hoc t-tests were computed in each of the revealed brain regions to examine the interactions $(* \mathrm{p}<0.05 ; * * \mathrm{p}<0.01 ; * * * \mathrm{p} \leq 0.001$, for more information on the t-tests see Table 5). 
in the vicinity of the left middle/dorsal anterior cingulate cortex (Figures 1 \& 2; Table 5). Importantly, the cluster in the left dorsal striatum overlapped to a great extent with the cluster revealed by the "Object category x Modality x Group" interaction from the between-subjects ANOVA (Figure 1).

Similarly, a whole-brain ANOVA was conducted for the non-smokers. A main effect of the factor "Object category" was found in the right parahippocampal gyrus, in the left middle temporal and inferior frontal gyrus, superior parietal lobe (SPL) and premotor cortex as well as in the bilateral superior temporal gyrus (Table 6). In all of these brain regions the fMRI response to control objects was lower as compared to smoking paraphernalia. The interaction "Object category x Modality" was significant only in the right middle temporal gyrus and the left superior temporal gyrus but in none of the regions showing the same interaction in smokers (Table 7).

Finally, the whole-brain ANCOVA revealed that the beta weights for the statistical contrast "VS -VC" were negatively correlated with the individual severity of nicotine dependence as indicated by the FTND scores in multiple brain regions in the right lingual gyrus, cuneus, lateral occipital complex, middle temporal gyrus, thalamus, posterior insula and supplementary motor area, left fusiform gyrus, inferior parietal lobule (IPL), posterior cingulate cortex, sensorimotor and premotor cortex and frontal eye fields as well as bilateral SPL (Table 8). In contrast there were no correlations between fMRI cue reactivity and FTND scores for the haptic modality.

\section{Discussion}

Addiction is a chronic disease marked by prominent changes in brain regions responsible for reward-related motivation, habitual and automatized behavior, as well as decision-making. It is commonly accepted that drug cues strongly activate the reward and habit circuits in the addicted brain, while the executive control regions, which exhibit a particular dysfunction in addicts, fail to exert control over these powerful and often impulsive signals, driving the addicted person to drugseeking and drug-taking behavior (Baler and Volkow, 2006; Koob and Volkow, 2010). However, in order to implement this knowledge effectively in the clinical routine, e.g. for the development of relapse predictors, reliable neural markers of these addiction-related brain dysfunctions have to be established. Several studies have demonstrated that neural cue reactivity can be used as a successful relapse predictor in addiction populations (Grusser et al., 2004; Kosten et al., 2006) but the consistency of the results from neural cue reactivity research is not very encouraging, indicating that the neural and behavioral responses to drug cues may depend on a number of factors (Conklin and Tiffany, 2002; Wilson et al., 2004). This is a crucial issue for addiction research, because if the biomarker used to assess the relapse risk is susceptible to various influences with unknown mechanisms, the prediction about a patient's relapse risk will be unreliable. Therefore, a thorough investigation of the factors which modulate neural cue reactivity is warranted.

In the present study we investigated the sensory modality in which cues are presented as one possible factor which may influence neural cue reactivity in 
nicotine addiction. In our within-subjects analysis we found that in smokers smoking-related stimuli induced a higher fMRI response than control objects depending on the sensory modality of the cues. The right PMd, left dorsal striatum and the right DLPFC showed a higher fMRI signal for smoking paraphernalia than control objects when the stimuli were presented haptically, while the reversed activation pattern with higher signals for visual control than smoking stimuli was found when the stimuli occurred in the visual modality (Figures $1 \& 2$ ). The opposite neural cue reactivity patterns - i.e. preference for the smoking cues over the control cues for the visual but not for the haptic modality - was observed in the right cerebellum (Figure 1). These findings were complemented by the between-subjects analysis which showed that the higher fMRI cue reactivity for haptic as compared to visual stimuli in the left dorsal striatum is seen only in smokers but not in nonsmokers. Importantly, for non-smokers we found no significant "Object category $\mathrm{x}$ Modality" interaction in any of the regions where the smokers' neural cue reactivity was modulated by sensory modality.

The involvement of these brain regions in the neural cue reactivity of smokers is not surprising, since their relevance for the development and maintenance of different addiction-related mechanisms is well-known. Cravingassociated activations of the DLPFC have been observed as a response to drug cues and linked to the contribution of the DLPFC to reward motivation and decision making (Wilson et al., 2004; Lee and Seo, 2007; Fecteau et al., 2010; Park et al., 2010). Interestingly, Wilson et al. (Wilson et al., 2004) suggested that the prefrontal cortex processes drug-cues in a context-dependent manner. More precisely, their review indicated that the perceived drug availability modulates the neural response to drug cues in the DLPFC. A similar mechanism might underlie the modalitydependent cue reactivity in the DLPFC observed in the present study. Since touching and manipulating smoking paraphernalia is very often directly followed by the act of smoking, haptic stimuli may imply a higher drug availability than visual cues. Thus, haptic input may be perceived by smokers as a more powerful predictor and trigger of smoking behavior in comparison to visual images, resulting in the prominent engagement of the DLPFC.

The dorsal striatum and PMd are essentially involved in the planning and execution of actions as well as the storage of action knowledge (Packard and Knowlton, 2002; Johnson-Frey, 2004). In the context of addiction they are of crucial importance for the establishment and expression of drug-associated habits and automatized action schemata responsible for the shift from initial drug consumption to a chronic and compulsive drug-use pattern (Tiffany, 1990; Everitt and Robbins, 2005; Yalachkov et al., 2009, 2010). Their function as mediators of drug-related habits and associated action knowledge explains why they are particularly responsive to haptic stimuli: the action of using smoking paraphernalia is strongly correlated with the respective haptic perception - for example lighting a cigarette always implies the haptic sensation and perception of the lighter and the cigarette. On the other hand, visual smoking cues are not always directly linked to the corresponding action, as one may for example see a cigarette in another person's hand. This could be the reason why in our study habit- and action-related brain regions such as the dorsal striatum and the PMd exhibited a pronounced smokingrelated neural cue reactivity for haptic but not for visual stimuli. 
One would expect that the cerebellum, which is not only responsive to haptic inputs (Naumer et al., 2010a) but also influences action patterns and stores tool use schemata (Imamizu et al., 2000; Imamizu et al., 2003), should respond to drug cues in a manner similar to the fMRI activation pattern of the dorsal striatum and the PMd. However, craving-associated activations of the cerebellum due to the presentation of visual drug cues have been reported (Grant et al., 1996) suggesting that the cerebellum may possess not only habit-related but also reward motivationassociated functions in the context of addiction.

Interestingly, the sensory modality-driven modulations of the neural cue reactivity in the above described brain regions were always in opposite directions for the two modalities used in the present study. While in the DLPFC, dorsal striatum and PMd haptic stimulation induced stronger fMRI responses for smoking than for control objects, the visual control cues elicited a higher fMRI signal than the visual smoking cues. The reversed pattern was found in the cerebellum. One might have expected that smoking-related stimuli induce a neural cue reactivity in both modalities which may be particularly strong in only one of them. Therefore we were surprised by the absence of any cue reactivity for the visual modality in the dorsal striatum, PMd and DLPFC. A careful examination of the stimuli used in the majority of the nicotine addiction studies may, however, reveal one possible explanation of these findings. For example, in an earlier study from our laboratory we used visual stimuli from the International Smoking Images Series [ISIS] (Gilbert and Rabinovich, 2006) and demonstrated cue reactivity in all of the brain regions described above (Yalachkov et al., 2009). These images which have been employed in a large number of nicotine addiction fMRI studies, are rather complex and salient, as they depict people smoking. Thus, a typical image from the ISIS shows not only smoking paraphernalia but also hands, faces, even whole bodies, other objects and further visual information about the environment. In contrast the visual stimuli used in the present study were rather simple, depicting only a single smoking-related object without any additional information. This might explain why we did not find any visual cue reactivity in the DLPFC, dorsal striatum and PMd: the visual smoking-related stimuli employed in the current experiment were perhaps not salient enough to induce a higher fMRI signal than the control objects. This explanation fits well to our findings, which demonstrated that there is neither a "Group x Category" interaction for the whole-brain ANOVA based on all subjects nor a "Category" main effect for the smokers in any of the reward and habit-related structures typically associated with addiction.

We found negative correlations between the severity of nicotine dependence measured by the FTND scores and the neural cue reactivity induced by visual stimuli in brain regions involved in the processing of sensory information as well as the allocation of attentional resources (Table 8). Several reports have indicated that lighter smokers show a greater attentional bias towards smoking cues than heavier smokers (Hogarth et al., 2003; Waters et al., 2003; Mogg et al., 2005; Yan et al., 2009). The present results might reflect the neural underpinnings of these observations.

The current study has some limitations which have to be addressed in future experiments. The smokers' group showed a relatively low level of nicotine dependence as indicated by the mean FTND scores. This could have limited the effect size and should be considered in future studies if one wanted to investigate the 
influence of sensory modality on neural cue reactivity in other brain regions which might be activated only in smokers with particularly high levels of dependence. Nevertheless, our experiment demonstrates that even in light or medium smokers, smoking-related neural cue reactivity is modulated by the sensory modality of the presented cue in brain regions involved in reward motivation, habits and decisionmaking such as the DLPFC, dorsal striatum, PMd and cerebellum.

To the best of our knowledge the current study is the first one to stress the importance of the sensory modality in which drug-associated stimuli are presented. Considering the sensory modality of the presented cues could have a considerable effect on the outcomes of neuroimaging investigations and exposure therapies of addiction. Our results suggest that the neural cue reactivity in the DLPFC, dorsal striatum and PMd is more pronounced for haptic than for visual stimuli, while the cerebellum is more responsive to visual than to haptic smoking-related cues. These findings could be used for the development of more reliable fMRI-specific biomarkers, more ecologically valid experimental designs, and more effective cueexposure therapies of addiction. 


\section{Tables}

Table 1. Between-subjects ANOVA: brain regions where a significant main effect of the factor "Group" was revealed (whole-brain ANOVA, $\mathrm{n}=30$ [15 smokers and 15 non-smokers], df $=29, \mathrm{p}<0.05$, corrected for multiple comparisons).

\begin{tabular}{|c|c|c|c|c|c|c|c|c|c|}
\hline & \multirow[b]{2}{*}{ Region } & \multicolumn{3}{|c|}{$\begin{array}{l}\text { Talairach } \\
\text { coordinates }\end{array}$} & \multirow[b]{2}{*}{$\begin{array}{l}\text { Nr. of } \\
\text { voxels }\end{array}$} & \multicolumn{2}{|c|}{$\begin{array}{l}\text { ANOVA (RFX of } \\
\text { Conjunction) }\end{array}$} & \multirow{2}{*}{$\begin{array}{l}\text { Beta } \\
\text { weights of } \\
\text { non- } \\
\text { smokers }\end{array}$} & \multirow{2}{*}{$\begin{array}{l}\text { Beta } \\
\text { weights of } \\
\text { smokers }\end{array}$} \\
\hline & & $\mathrm{x}$ & $\mathrm{y}$ & $\mathrm{z}$ & & Average & Average & & \\
\hline \multirow{9}{*}{ 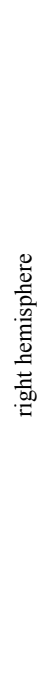 } & lingual gyrus & 21 & -74 & 0 & 623 & 9.19 & 0.006 & 0.250 & -0.031 \\
\hline & inferior frontal gyrus & 55 & 5 & 13 & 966 & 9.12 & 0.006 & 0.339 & 0.554 \\
\hline & middle occipital gyrus & 34 & -81 & 16 & 450 & 9.57 & 0.005 & 0.216 & -0.064 \\
\hline & $\begin{array}{l}\text { superior temporal gyrus, } \\
\text { BA22 }\end{array}$ & 50 & -56 & 14 & 411 & 8.57 & 0.007 & 0.365 & 0.096 \\
\hline & posterior cingulate gyrus & 11 & -53 & 29 & 237 & 9.24 & 0.006 & 0.043 & -0.115 \\
\hline & precuneus & 3 & -61 & 38 & 470 & 8.95 & 0.006 & 0.222 & 0.012 \\
\hline & premotor cortex, subgyral & 26 & 6 & 40 & 779 & 9.39 & 0.005 & 0.155 & -0.063 \\
\hline & medial frontal gyrus & 1 & 21 & 44 & 1843 & 9.86 & 0.005 & 0.214 & 0.019 \\
\hline & $\begin{array}{l}\text { pre-supplementary/ } \\
\text { supplementary motor area }\end{array}$ & 4 & -17 & 55 & 562 & 9.51 & 0.005 & 0.150 & 0.530 \\
\hline \multirow{6}{*}{ 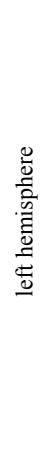 } & fusiform gyrus & -39 & -38 & -12 & 314 & 9.29 & 0.006 & 0.392 & 0.156 \\
\hline & cuneus & -6 & -56 & 8 & 489 & 8.56 & 0.007 & -0.076 & -0.326 \\
\hline & $\begin{array}{l}\text { middle temporal } \\
\text { gyrus/BA19 }\end{array}$ & -35 & -82 & 22 & 1559 & 10.60 & 0.004 & 0.158 & -0.169 \\
\hline & middle temporal gyrus & -38 & -59 & 17 & 1142 & 10.19 & 0.005 & 0.275 & -0.094 \\
\hline & $\begin{array}{l}\text { white matter, vicinity } \\
\text { of anterior cingulate } \\
\text { cortex }\end{array}$ & -18 & -9 & 37 & 223 & 8.79 & 0.006 & -0.019 & 0.088 \\
\hline & $\begin{array}{l}\text { middle frontal gyrus } \\
\text { BA8/9 }\end{array}$ & -47 & 8 & 40 & 1395 & 9.56 & 0.005 & 0.419 & 0.136 \\
\hline
\end{tabular}


Chapter 4

Table 2. Between-subjects ANOVA: brain regions where the interaction "Object category x Group" was significant (whole-brain ANOVA, $\mathrm{n}=30$ [15 smokers and 15 non-smokers], $\mathrm{df}=29, \mathrm{p}<0.05$, corrected for multiple comparisons).

\begin{tabular}{|c|c|c|c|c|c|c|c|c|c|c|c|}
\hline \multirow{2}{*}{\multicolumn{2}{|c|}{ Region }} & \multicolumn{3}{|c|}{$\begin{array}{c}\text { Talairach } \\
\text { coordinates }\end{array}$} & \multirow[b]{2}{*}{$\begin{array}{l}\mathrm{Nr} \text { of } \\
\text { voxels }\end{array}$} & \multicolumn{2}{|c|}{$\begin{array}{l}\text { ANOVA (RFX of } \\
\text { Conjunction) }\end{array}$} & \multicolumn{2}{|c|}{$\begin{array}{l}\text { Beta weights } \\
\text { "Control } \\
\text { objects" }\end{array}$} & \multicolumn{2}{|c|}{$\begin{array}{l}\text { Beta weights } \\
\text { "Smoking } \\
\text { paraphernalia" }\end{array}$} \\
\hline & & $\mathrm{x}$ & $\mathrm{y}$ & $\mathrm{z}$ & & $\begin{array}{l}\text { Average } \\
\text { F-value }\end{array}$ & Average & $\begin{array}{l}\text { Non- } \\
\text { smokers }\end{array}$ & Smokers & $\begin{array}{l}\text { Non- } \\
\text { smokers }\end{array}$ & Smokers \\
\hline \multirow{2}{*}{ 莺离 } & $\begin{array}{l}\text { middle } \\
\text { temporal } \\
\text { gyrus }\end{array}$ & 55 & -10 & -7 & 217 & 8.07 & 0.008 & -0.202 & -0.113 & -0.066 & -0.203 \\
\hline & $\begin{array}{l}\text { lingual } \\
\text { gyrus }\end{array}$ & 12 & -82 & 5 & 4340 & 11.27 & 0.004 & -0.047 & -0.071 & 0.033 & -0.195 \\
\hline \multirow{2}{*}{ 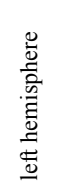 } & $\begin{array}{l}\text { lingual } \\
\text { gyrus }\end{array}$ & -22 & -75 & -8 & 316 & 9.07 & 0.006 & -0.040 & -0.087 & 0.014 & -0.177 \\
\hline & $\begin{array}{l}\text { lingual } \\
\text { gyrus }\end{array}$ & -12 & -89 & 1 & 292 & 8.72 & 0.007 & 0.173 & 0.126 & 0.214 & 0.017 \\
\hline
\end{tabular}

Table 3. Between-subjects ANOVA: brain regions where the interaction "Object category $\mathrm{x}$ Modality $\mathrm{x}$ Group" was significant (whole-brain ANOVA, $\mathrm{n}=30$ [15 smokers and 15 non-smokers], $\mathrm{df}=29, \mathrm{p}<$ 0.05 , corrected for multiple comparisons).

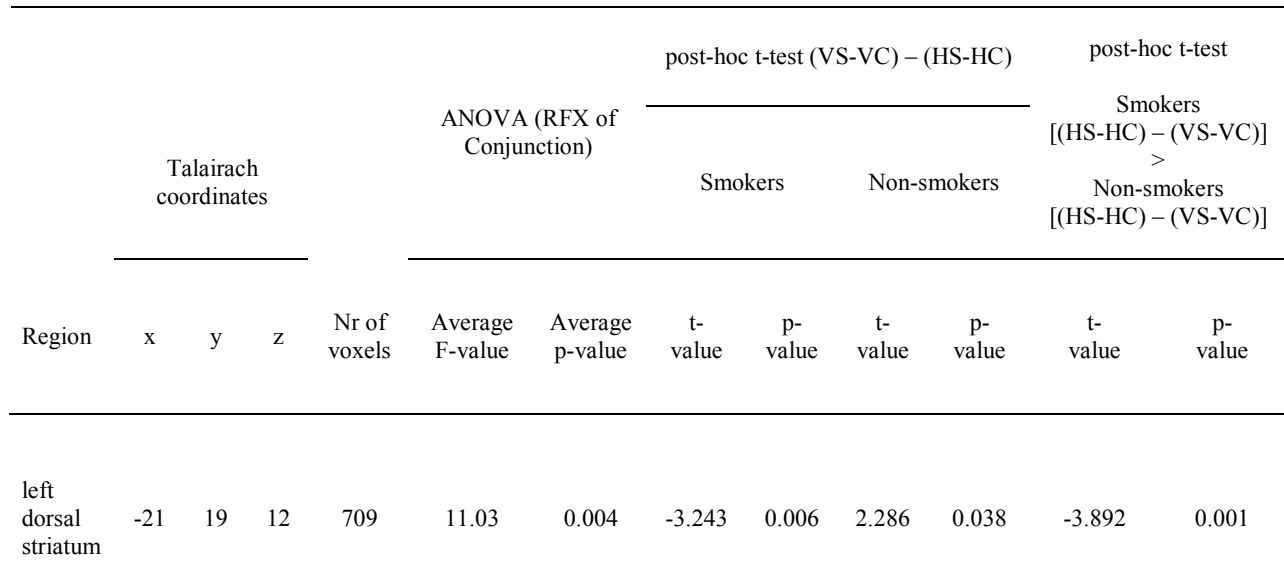


Table 4. Within-subjects ANOVA: brain regions where a significant main effect of the factor "Object category" was revealed (whole-brain ANOVA, $\mathrm{n}=15$ smokers, $\mathrm{df}=14, \mathrm{p}<0.05$, corrected for multiple comparisons).

\begin{tabular}{|c|c|c|c|c|c|c|c|c|c|}
\hline & \multirow[b]{2}{*}{ Region } & \multicolumn{3}{|c|}{$\begin{array}{c}\text { Talairach } \\
\text { coordinates }\end{array}$} & \multirow[b]{2}{*}{$\mathrm{Nr}$ of voxels } & \multicolumn{2}{|c|}{$\begin{array}{l}\text { ANOVA (RFX of } \\
\text { Conjunction) }\end{array}$} & \multirow[b]{2}{*}{$\begin{array}{c}\text { Beta weights } \\
\text { „Control“" }\end{array}$} & \multirow[b]{2}{*}{$\begin{array}{c}\text { Beta weights } \\
\text { „Smoke“ }\end{array}$} \\
\hline & & $\mathrm{x}$ & $\mathrm{y}$ & $\mathrm{Z}$ & & $\begin{array}{l}\text { Average } \\
\text { F-value }\end{array}$ & $\begin{array}{l}\text { Average } \\
\text { p-value }\end{array}$ & & \\
\hline \multirow{3}{*}{ 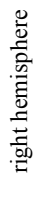 } & lingual gyrus & 22 & -81 & -2 & 6007 & 14.69 & 0.004 & 0.276 & 0.136 \\
\hline & cuneus & 18 & -70 & 7 & 241 & 10.04 & 0.007 & -0.022 & -0.111 \\
\hline & $\begin{array}{l}\text { precentral } \\
\text { gyrus, BA4 }\end{array}$ & 61 & -10 & 29 & 442 & 12.30 & 0.005 & 0.205 & 0.067 \\
\hline$\frac{0}{0}$ & lingual gyrus & -29 & -75 & -7 & 4301 & 13.39 & 0.004 & 0.452 & 0.319 \\
\hline 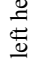 & cuneus & -13 & -79 & 11 & 435 & 10.52 & 0.006 & -0.152 & -0.255 \\
\hline
\end{tabular}

Table 5. Within-subjects ANOVA: brain regions where the interaction "Object category x Modality" was significant (whole-brain ANOVA, $\mathrm{n}=15$ smokers, $\mathrm{df}=14, \mathrm{p}<0.05$, corrected for multiple comparisons).

\begin{tabular}{|c|c|c|c|c|c|c|c|c|c|c|c|c|c|}
\hline & \multirow[b]{2}{*}{ Region } & \multicolumn{3}{|c|}{$\begin{array}{l}\text { Talairach } \\
\text { coordinates }\end{array}$} & \multirow[b]{2}{*}{$\begin{array}{c}\mathrm{Nr} \text { of } \\
\text { voxels }\end{array}$} & \multicolumn{2}{|c|}{$\begin{array}{c}\text { RFX of } \\
\text { Conjunction }\end{array}$} & \multicolumn{2}{|c|}{$\begin{array}{l}\text { Beta weights } \\
\text { "Control" }\end{array}$} & \multicolumn{2}{|c|}{$\begin{array}{l}\text { Beta weights } \\
\text { „Smoke“ }\end{array}$} & \multicolumn{2}{|c|}{$\begin{array}{c}\text { post-hoc } \\
\text { t-test } \\
\text { (VS-VC) - } \\
\text { (HS-HC) }\end{array}$} \\
\hline & & $\mathrm{x}$ & y & $\mathrm{z}$ & & $\begin{array}{l}\text { Average } \\
\text { F-value }\end{array}$ & $\begin{array}{l}\text { Average } \\
\text { p-value }\end{array}$ & V & $\mathrm{H}$ & $\mathrm{V}$ & $\mathrm{H}$ & t-value & $\mathrm{p}$-value \\
\hline \multirow{6}{*}{ 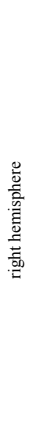 } & cerebellum & 31 & -70 & -27 & 278 & 10.53 & 0.006 & 0.193 & 0.213 & 0.336 & 0.177 & 4.563 & $<0.001$ \\
\hline & $\begin{array}{l}\text { medial temporal lobe } \\
\text { (white matter, vicinity of } \\
\text { parahippocampal gyrus) }\end{array}$ & 39 & -15 & -21 & 648 & 11.98 & 0.005 & -0.036 & 0.089 & 0.092 & -0.004 & 4.951 & $<0.001$ \\
\hline & orbitofrontal cortex & 4 & 32 & -16 & 299 & 9.92 & 0.007 & -0.250 & 0.009 & -0.060 & -0.114 & 3.283 & 0.005 \\
\hline & $\begin{array}{l}\text { anterior prefrontal cortex } \\
\text { (white matter, vicinity of } \\
\text { BA10) }\end{array}$ & 35 & 49 & -8 & 409 & 11.17 & 0.006 & -0.069 & 0.146 & 0.032 & -0.063 & 4.004 & 0.001 \\
\hline & $\begin{array}{l}\text { dorsolateral prefrontal } \\
\text { cortex }\end{array}$ & 44 & 31 & 41 & 282 & 10.23 & 0.007 & 0.269 & 0.175 & 0.188 & 0.245 & -2.210 & 0.044 \\
\hline & dorsal premotor cortex & 19 & -12 & 68 & 257 & 10.51 & 0.007 & 0.365 & 0.545 & 0.284 & 0.633 & -3.296 & 0.005 \\
\hline \multirow{3}{*}{ 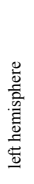 } & middle occipital gyrus & -40 & -88 & 6 & 263 & 10.58 & 0.006 & 0.219 & -0.055 & 0.328 & -0.135 & 3.322 & 0.005 \\
\hline & dorsal striatum & -19 & 20 & 13 & 619 & 11.13 & 0.006 & 0.160 & 0.073 & 0.010 & 0.123 & -3.969 & 0.001 \\
\hline & $\begin{array}{l}\text { white matter, vicinity of } \\
\text { middle/dorsal anterior } \\
\text { cingulate gyrus }\end{array}$ & -12 & -2 & 29 & 246 & 10.81 & 0.006 & 0.096 & 0.000 & 0.003 & 0.072 & -4.370 & 0.001 \\
\hline
\end{tabular}


Table 6. Within-subjects ANOVA: brain regions where a significant main effect of the factor "Object category" was revealed (whole-brain ANOVA, $\mathrm{n}=15$ non-smokers, $\mathrm{df}=14, \mathrm{p}<0.05$, corrected for multiple comparisons).

\begin{tabular}{|c|c|c|c|c|c|c|c|c|c|}
\hline \multirow{2}{*}{\multicolumn{2}{|c|}{ Region }} & \multicolumn{3}{|c|}{$\begin{array}{l}\text { Talairach } \\
\text { coordinates }\end{array}$} & \multirow[b]{2}{*}{$\begin{array}{l}\mathrm{Nr} \text { of } \\
\text { voxels }\end{array}$} & \multicolumn{2}{|c|}{$\begin{array}{l}\text { ANOVA (RFX of } \\
\text { Conjunction) }\end{array}$} & \multirow{2}{*}{$\begin{array}{c}\text { Beta } \\
\text { weights } \\
\text { „Control“ }\end{array}$} & \multirow[b]{2}{*}{$\begin{array}{l}\text { Beta weights } \\
\text { „Smoke“" }\end{array}$} \\
\hline & & $\mathrm{x}$ & $\mathrm{y}$ & $\mathrm{z}$ & & $\begin{array}{c}\text { Average } \\
\text { F-value }\end{array}$ & $\begin{array}{l}\text { Average } \\
\text { p-value }\end{array}$ & & \\
\hline \multirow{3}{*}{ 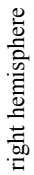 } & parahippocampal gyrus & 30 & -30 & -22 & 606 & 11.82 & 0.005 & 0.345 & 0.484 \\
\hline & middle temporal gyrus & 57 & -11 & -6 & 667 & 10.42 & 0.006 & -0.197 & -0.032 \\
\hline & superior temporal gyrus & 47 & -61 & 19 & 2157 & 11.00 & 0.006 & 0.051 & 0.226 \\
\hline \multirow{12}{*}{ 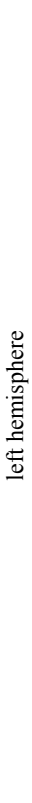 } & middle temporal gyrus & -53 & -12 & -14 & 1766 & 10.66 & 0.006 & -0.223 & -0.066 \\
\hline & middle temporal gyrus & -59 & -43 & -3 & 635 & 10.04 & 0.007 & 0.064 & 0.225 \\
\hline & $\begin{array}{l}\text { superior temporal gyrus, } \\
\text { BA38 }\end{array}$ & -40 & 16 & -20 & 1215 & 11.85 & 0.005 & -0.226 & -0.066 \\
\hline & superior temporal gyrus & -50 & -60 & 21 & 2093 & 13.04 & 0.004 & 0.053 & 0.214 \\
\hline & inferior frontal gyrus & -45 & 24 & -10 & 271 & 11.64 & 0.005 & -0.173 & -0.046 \\
\hline & inferior frontal gyrus & -45 & 35 & 0 & 376 & 10.56 & 0.006 & -0.086 & 0.032 \\
\hline & inferior frontal gyrus & -43 & 14 & 23 & 508 & 10.14 & 0.007 & 0.086 & 0.196 \\
\hline & $\begin{array}{l}\text { frontal lobe, white matter, } \\
\text { adjacent to insula }\end{array}$ & -28 & -11 & 25 & 261 & 11.10 & 0.006 & 0.057 & -0.041 \\
\hline & superior parietal lobule & -28 & -72 & 48 & 746 & 10.48 & 0.006 & 0.261 & 0.407 \\
\hline & premotor cortex, BA6 & -36 & -4 & 44 & 540 & 11.46 & 0.005 & 0.268 & 0.388 \\
\hline & superior frontal gyrus & -26 & 17 & 46 & 2043 & 11.34 & 0.005 & -0.142 & -0.003 \\
\hline & $\begin{array}{l}\text { superior frontal gyrus, } \\
\text { BA8 }\end{array}$ & -9 & 44 & 49 & 540 & 10.07 & 0.007 & -0.232 & -0.108 \\
\hline $\begin{array}{l}\bar{\pi} \\
\frac{\pi}{0} \\
\frac{\pi}{30}\end{array}$ & orbitofrontal cortex & 2 & 32 & -11 & 638 & 10.61 & 0.006 & -0.284 & -0.142 \\
\hline
\end{tabular}


Table 7. Within-subjects ANOVA: brain regions where the interaction "Object category x Modality" was significant (whole-brain ANOVA, $\mathrm{n}=15$ non-smokers, $\mathrm{df}=14, \mathrm{p}<0.05$, corrected for multiple comparisons).

\begin{tabular}{|c|c|c|c|c|c|c|c|c|c|c|}
\hline \multirow[b]{2}{*}{ Region } & \multicolumn{3}{|c|}{$\begin{array}{c}\text { Talairach } \\
\text { coordinates }\end{array}$} & \multirow{2}{*}{$\begin{array}{l}\mathrm{Nr} \text { of } \\
\text { voxels }\end{array}$} & \multicolumn{2}{|c|}{$\begin{array}{c}\text { ANOVA (RFX of } \\
\text { Conjunction) }\end{array}$} & \multicolumn{2}{|c|}{$\begin{array}{c}\text { Beta weights } \\
\text { „Control“" }\end{array}$} & \multicolumn{2}{|c|}{$\begin{array}{l}\text { Beta weights } \\
\text { „Smoke“" }\end{array}$} \\
\hline & $\mathrm{x}$ & $\mathrm{y}$ & z & & $\begin{array}{l}\text { Average } \\
\text { F-value }\end{array}$ & $\begin{array}{l}\text { Average } \\
\text { p-value }\end{array}$ & V & $\mathrm{H}$ & V & $\mathrm{H}$ \\
\hline $\begin{array}{l}\text { right middle } \\
\text { temporal gyrus }\end{array}$ & 53 & -23 & -13 & 312 & 10.07 & 0.007 & 0.008 & 0.156 & 0.164 & 0.068 \\
\hline $\begin{array}{l}\text { left superior } \\
\text { temporal gyrus }\end{array}$ & -46 & -35 & 15 & 523 & 12.16 & 0.005 & -0.015 & 0.288 & 0.121 & 0.261 \\
\hline
\end{tabular}

Table 8. Brain regions where the beta weights from the visual modality-specific contrast "smoking paraphernalia - control objects" (VS - VC) were correlated with the FTND scores (whole-brain ANCOVA; $\mathrm{n}=15$ smokers; $\mathrm{df}=13 ; \mathrm{r} \geq+/-0.642 ; \mathrm{p}<0.05$, corrected for multiple comparisons).

\begin{tabular}{|c|c|c|c|c|c|c|c|}
\hline & \multirow[b]{2}{*}{ Region } & \multicolumn{3}{|c|}{$\begin{array}{l}\text { Talairach } \\
\text { coordinates }\end{array}$} & \multirow[b]{2}{*}{$\begin{array}{l}\text { Nr. of } \\
\text { voxels }\end{array}$} & \multicolumn{2}{|c|}{ ANCOVA } \\
\hline & & $\mathrm{x}$ & $\mathrm{y}$ & $\mathrm{z}$ & & $\begin{array}{l}\text { Average } \\
\text { r-value }\end{array}$ & $\begin{array}{l}\text { Average } \\
\text { p-value }\end{array}$ \\
\hline \multirow{8}{*}{ 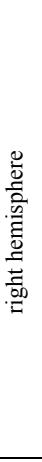 } & lingual gyrus & 15 & -88 & -16 & 1303 & -0.69 & 0.005 \\
\hline & middle temporal gyrus & 55 & -6 & -19 & 472 & -0.70 & 0.005 \\
\hline & $\begin{array}{l}\text { lateral occipital } \\
\text { complex }\end{array}$ & 45 & -62 & -4 & 1101 & -0.68 & 0.006 \\
\hline & cuneus & 20 & -89 & 5 & 838 & -0.68 & 0.006 \\
\hline & thalamus & 10 & -25 & 1 & 775 & -0.68 & 0.006 \\
\hline & posterior insula & 39 & -34 & 17 & 386 & -0.66 & 0.008 \\
\hline & $\begin{array}{l}\text { superior parietal } \\
\text { lobule }\end{array}$ & 21 & -54 & 59 & 3639 & -0.71 & 0.004 \\
\hline & $\begin{array}{l}\text { supplementary motor } \\
\text { area }\end{array}$ & 2 & -15 & 61 & 970 & -0.68 & 0.006 \\
\hline \multirow{7}{*}{ 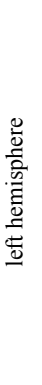 } & fusiform gyrus & -43 & -72 & -21 & 488 & -0.67 & 0.007 \\
\hline & inferior parietal lobule & -41 & -30 & 33 & 344 & -0.67 & 0.007 \\
\hline & $\begin{array}{l}\text { posterior cingulate } \\
\text { cortex }\end{array}$ & -2 & -28 & 40 & 938 & -0.66 & 0.008 \\
\hline & sensorimotor cortex & -36 & -15 & 48 & 1272 & -0.67 & 0.006 \\
\hline & $\begin{array}{l}\text { superior frontal gyrus, } \\
\text { BA8 }\end{array}$ & -22 & 25 & 47 & 421 & -0.66 & 0.007 \\
\hline & $\begin{array}{l}\text { superior parietal } \\
\text { lobule }\end{array}$ & -9 & -48 & 67 & 857 & -0.67 & 0.006 \\
\hline & premotor cortex & -16 & -16 & 67 & 1721 & -0.69 & 0.005 \\
\hline
\end{tabular}


Table 9. Between-subjects ANOVA: brain regions where a significant main effect of the factor "Object category" was revealed (whole-brain ANOVA, $\mathrm{n}=30$ [15 smokers and 15 non-smokers], df = 29, $\mathrm{p}<$ 0.05 , corrected for multiple comparisons).

\begin{tabular}{|c|c|c|c|c|c|c|c|c|c|}
\hline \multirow{2}{*}{\multicolumn{2}{|c|}{ Region }} & \multicolumn{3}{|c|}{$\begin{array}{l}\text { Talairach } \\
\text { coordinates }\end{array}$} & \multirow{2}{*}{$\begin{array}{l}\mathrm{Nr} \text { of } \\
\text { voxels }\end{array}$} & \multicolumn{2}{|c|}{$\begin{array}{l}\text { ANOVA (RFX of } \\
\text { Conjunction) }\end{array}$} & \multirow[b]{2}{*}{$\begin{array}{l}\text { Beta weights } \\
\text { „Control“" }\end{array}$} & \multirow[b]{2}{*}{$\begin{array}{l}\text { Beta weights } \\
\text { „Smoke“ }\end{array}$} \\
\hline & & $\mathrm{x}$ & $\mathrm{y}$ & $\mathrm{z}$ & & $\begin{array}{c}\text { Average } \\
\text { F-value }\end{array}$ & $\begin{array}{l}\text { Average } \\
\text { p-value }\end{array}$ & & \\
\hline \multirow{2}{*}{ 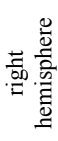 } & $\begin{array}{l}\text { occipital/temporal } \\
\text { cortex }\end{array}$ & 50 & -66 & 17 & 700 & 9.06 & 0.006 & -0.049 & 0.075 \\
\hline & motor cortex & 63 & -9 & 29 & 309 & 9.66 & 0.005 & 0.123 & 0.039 \\
\hline \multirow{7}{*}{ 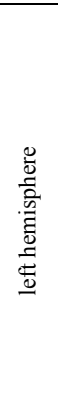 } & $\begin{array}{l}\text { middle temporal } \\
\text { gyrus, BA21 }\end{array}$ & -56 & -22 & -14 & 987 & 9.42 & 0.005 & -0.107 & 0.013 \\
\hline & $\begin{array}{l}\text { subcallosal gyrus, } \\
\text { subgyral }\end{array}$ & -22 & 16 & -10 & 421 & 9.26 & 0.006 & -0.088 & 0 \\
\hline & middle temporal gyrus & -59 & -42 & -6 & 396 & 8.40 & 0.007 & 0.004 & 0.123 \\
\hline & $\begin{array}{l}\text { inferior/middle frontal } \\
\text { gyrus }\end{array}$ & -44 & 34 & -4 & 959 & 8.70 & 0.007 & -0.104 & -0.005 \\
\hline & inferior frontal gyrus & -46 & 14 & 19 & 651 & 8.80 & 0.007 & 0.071 & 0.169 \\
\hline & $\begin{array}{l}\text { occipital/temporal } \\
\text { cortex }\end{array}$ & -49 & -64 & 24 & 3721 & 9.52 & 0.005 & -0.014 & 0.094 \\
\hline & $\begin{array}{l}\text { superior frontal gyrus, } \\
\text { white matter }\end{array}$ & -14 & 21 & 48 & 282 & 8.862 & 0.006 & -0.158 & -0.056 \\
\hline
\end{tabular}

Table 10. Between-subjects ANOVA: brain regions where a significant main effect of the factor "Modality" was revealed (whole-brain ANOVA, $\mathrm{n}=30$ [15 smokers and 15 non-smokers], df $=29, \mathrm{p}<$ 0.05 , corrected for multiple comparisons).

\begin{tabular}{|c|c|c|c|c|c|c|c|}
\hline & \multirow{2}{*}{ Region } & \multicolumn{3}{|c|}{$\begin{array}{c}\text { Talairach } \\
\text { coordinates }\end{array}$} & \multirow[b]{2}{*}{ Nr of voxels } & \multicolumn{2}{|c|}{ ANOVA (RFX of Conjunction) } \\
\hline & & $\mathrm{X}$ & $\mathrm{y}$ & Z & & Average & Average \\
\hline \multirow{4}{*}{ 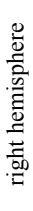 } & occipitotemporal cortex & 25 & -70 & 4 & 44337 & 69.80 & $<0.001$ \\
\hline & thalamus & 9 & -18 & 7 & 2402 & 28.55 & $<0.001$ \\
\hline & $\begin{array}{l}\text { paracentral lobule, } \\
\text { white matter }\end{array}$ & 14 & -29 & 57 & 54 & 21 & $<0.001$ \\
\hline & premotor cortex & 44 & -2 & 37 & 10 & 19.64 & $<0.001$ \\
\hline \multirow{5}{*}{ 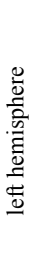 } & occipitotemporal cortex & -30 & -76 & 6 & 25139 & 56.22 & $<0.001$ \\
\hline & amygdala & -23 & -1 & -10 & 91 & 20.97 & $<0.001$ \\
\hline & cerebellum, vermis & -1 & -63 & -6 & 246 & 20.54 & $<0.001$ \\
\hline & thalamus & -13 & -20 & 7 & 1918 & 29.92 & $<0.001$ \\
\hline & temporal cortex & -50 & -59 & 7 & 3127 & 26.41 & $<0.001$ \\
\hline \multirow{2}{*}{$\begin{array}{l}\frac{\pi}{0} \\
\frac{0}{0} \\
\frac{\pi}{0}\end{array}$} & sensorimotor cortex & -1 & -28 & 36 & 125925 & 49.09 & $<0.001$ \\
\hline & posterior cingulate & 0 & -26 & 40 & 1552 & 23.19 & $<0.001$ \\
\hline
\end{tabular}


Table 11. Between-subjects ANOVA: brain regions where the interaction "Object category x Modality" was significant (whole-brain ANOVA, $\mathrm{n}=30$ [15 smokers and 15 non-smokers], $\mathrm{df}=29, \mathrm{p}<0.05$, corrected for multiple comparisons).

\begin{tabular}{|c|c|c|c|c|c|c|c|}
\hline & \multirow[b]{2}{*}{ Region } & \multicolumn{3}{|c|}{$\begin{array}{c}\text { Talairach } \\
\text { coordinates }\end{array}$} & \multirow{2}{*}{$\begin{array}{c}\mathrm{Nr} \text { of } \\
\text { voxels }\end{array}$} & \multicolumn{2}{|c|}{ ANOVA (RFX of Conjunction) } \\
\hline & & $\mathrm{X}$ & $\mathrm{y}$ & $\mathrm{z}$ & & Average & Average \\
\hline \multirow{3}{*}{ 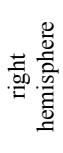 } & inferior/middle temporal gyrus & 52 & -22 & -13 & 1775 & 10.27 & 0.005 \\
\hline & $\begin{array}{l}\text { superior temporal gyrus/ } \\
\text { posterior insula }\end{array}$ & 41 & 10 & -14 & 738 & 9.75 & 0.005 \\
\hline & orbitofrontal cortex & 5 & 31 & -12 & 302 & 8.87 & 0.006 \\
\hline \multirow{2}{*}{ 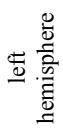 } & orbitofrontal cortex & -6 & 44 & -9 & 1128 & 9.65 & 0.005 \\
\hline & middle occipital gyrus & -40 & -87 & 4 & 302 & 9.34 & 0.005 \\
\hline
\end{tabular}

Table 12. Between-subjects ANOVA: brain regions where the interaction "Modality x Group" was significant (whole-brain ANOVA, $\mathrm{n}=30$ [15 smokers and 15 non-smokers], $\mathrm{df}=29, \mathrm{p}<0.05$, corrected for multiple comparisons).

\begin{tabular}{|c|c|c|c|c|c|c|c|}
\hline & \multirow[b]{2}{*}{ Region } & \multicolumn{3}{|c|}{$\begin{array}{l}\text { Talairach } \\
\text { coordinates }\end{array}$} & \multirow[b]{2}{*}{$\begin{array}{l}\mathrm{Nr} \text { of } \\
\text { voxels }\end{array}$} & \multicolumn{2}{|c|}{ ANOVA (RFX of Conjunction) } \\
\hline & & $\mathrm{x}$ & $\mathrm{y}$ & $\mathrm{z}$ & & $\begin{array}{l}\text { Average } \\
\text { F-value }\end{array}$ & $\begin{array}{l}\text { Average } \\
\text { p-value }\end{array}$ \\
\hline \multirow{9}{*}{ 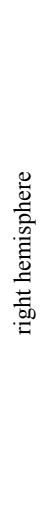 } & lingual gyrus & 17 & -78 & -2 & 337 & 8.84 & 0.006 \\
\hline & $\begin{array}{l}\text { middle occipital gyrus, } \\
\text { white matter }\end{array}$ & 36 & -61 & 1 & 560 & 8.62 & 0.007 \\
\hline & cuneus & 21 & -73 & 13 & 740 & 9.50 & 0.005 \\
\hline & precuneus & 28 & -67 & 27 & 242 & 8.50 & 0.007 \\
\hline & cuneus, BA19 & 10 & -86 & 31 & 233 & 9.69 & 0.005 \\
\hline & $\begin{array}{l}\text { cingulate gyrus, white } \\
\text { matter }\end{array}$ & 10 & -13 & 42 & 284 & 9.68 & 0.005 \\
\hline & $\begin{array}{l}\text { superior frontal gyrus, } \\
\text { BA8 }\end{array}$ & 22 & 21 & 43 & 229 & 8.89 & 0.006 \\
\hline & superior parietal lobule & 31 & -60 & 47 & 2521 & 10.70 & 0.004 \\
\hline & precentral gyrus & 30 & -20 & 48 & 260 & 8.87 & 0.006 \\
\hline \multirow{6}{*}{ 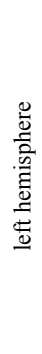 } & lateral occipital complex & -44 & -71 & -3 & 1403 & 10.26 & 0.004 \\
\hline & ventral striatum & -8 & 16 & 7 & 597 & 10.91 & 0.004 \\
\hline & $\begin{array}{l}\text { middle occipital gyrus, } \\
\text { BA19 }\end{array}$ & -29 & -84 & 20 & 784 & 9.07 & 0.006 \\
\hline & medial frontal gyrus & -1 & 39 & 34 & 1077 & 8.60 & 0.007 \\
\hline & $\begin{array}{l}\text { posterior cingulate gyrus, } \\
\text { white matter }\end{array}$ & -19 & -30 & 34 & 247 & 9.17 & 0.006 \\
\hline & $\begin{array}{l}\text { superior frontal gyrus, } \\
\text { BA8 }\end{array}$ & -35 & 17 & 49 & 585 & 9.08 & 0.006 \\
\hline
\end{tabular}


Chapter 4

Table 13. Within-subjects ANOVA: brain regions where a significant main effect of the factor "Modality" was revealed (whole-brain ANOVA, $\mathrm{n}=15$ smokers, $\mathrm{df}=14, \mathrm{p}<0.05$, corrected for multiple comparisons).

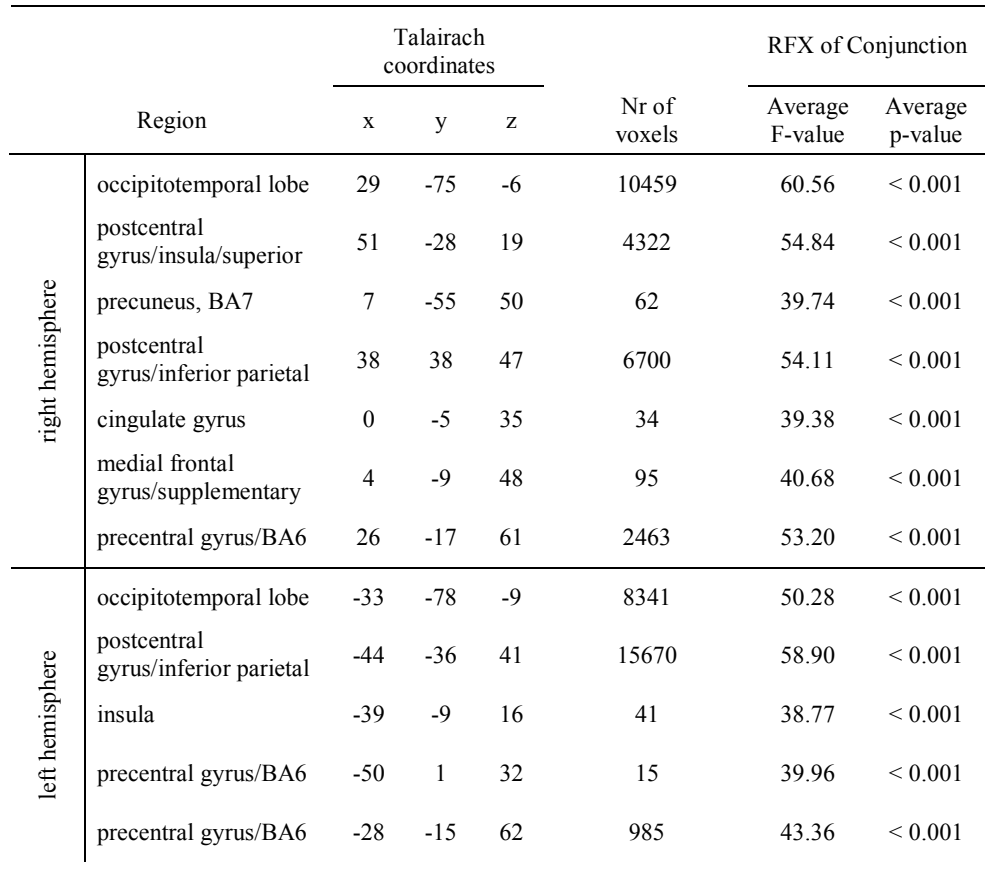


Table 14. Within-subjects ANOVA: brain regions where a significant main effect of the factor "Modality" was revealed (whole-brain ANOVA, $\mathrm{n}=15$ non-smokers, $\mathrm{df}=14, \mathrm{p}<0.05$, corrected for multiple comparisons).

\begin{tabular}{|c|c|c|c|c|c|c|c|}
\hline \multirow{2}{*}{\multicolumn{2}{|c|}{ Region }} & \multicolumn{3}{|c|}{$\begin{array}{l}\text { Talairach } \\
\text { coordinates }\end{array}$} & \multirow[b]{2}{*}{$\begin{array}{l}\mathrm{Nr} \text { of } \\
\text { voxels }\end{array}$} & \multicolumn{2}{|c|}{ ANOVA (RFX of Conjunction) } \\
\hline & & $\mathrm{x}$ & $\mathrm{y}$ & $\mathrm{z}$ & & $\begin{array}{l}\text { Average } \\
\text { F-value }\end{array}$ & $\begin{array}{c}\text { Average } \\
\text { p-value }\end{array}$ \\
\hline \multirow{5}{*}{ 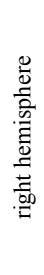 } & occipitotemporal cortex & 26 & -76 & 3 & 32055 & 71.03 & $<0.001$ \\
\hline & $\begin{array}{l}\text { posterior middle temporal } \\
\text { gyrus }\end{array}$ & 47 & -58 & 9 & 7 & 35.86 & $<0.001$ \\
\hline & $\begin{array}{l}\text { posterior middle temporal } \\
\text { gyrus }\end{array}$ & 50 & -50 & 12 & 15 & 35.85 & $<0.001$ \\
\hline & sensorimotor cortex & 43 & -30 & 40 & 11588 & 50.38 & $<0.001$ \\
\hline & precentral gyrus, BA6 & 30 & -11 & 58 & 127 & 37.29 & $<0.001$ \\
\hline \multirow{10}{*}{ 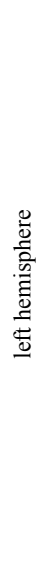 } & amygdala & -28 & 0 & -12 & 135 & 44.53 & $<0.001$ \\
\hline & cerebellum & -25 & -48 & -23 & 102 & 39.92 & $<0.001$ \\
\hline & occipitotemporal cortex & -34 & -79 & 1 & 20410 & 69.00 & $<0.001$ \\
\hline & insula & -39 & -10 & 5 & 465 & 41.39 & $<0.001$ \\
\hline & thalamus & -13 & -20 & 8 & 39 & 36.24 & $<0.001$ \\
\hline & $\begin{array}{l}\text { posterior middle temporal } \\
\text { gyrus }\end{array}$ & -51 & -60 & 9 & 58 & 36.79 & $<0.001$ \\
\hline & insula & -39 & -6 & 16 & 132 & 36.64 & $<0.001$ \\
\hline & sensorimotor cortex & -48 & -31 & 40 & 14162 & 59.13 & $<0.001$ \\
\hline & precentral gyrus, BA6 & -30 & -15 & 59 & 1162 & 42.35 & $<0.001$ \\
\hline & superior frontal gyrus, BA6 & -20 & -8 & 63 & 10 & 35.98 & $<0.001$ \\
\hline
\end{tabular}





\section{Chapter 5}

\section{Smoking experience modulates the cortical integration of vision and haptics}

\section{Based on:}

Yalachkov Y, Kaiser J, Goerres A, Seehaus A, Naumer MJ. Smoking experience modulates the cortical integration of vision and haptics. (Cerebral Cortex, under review) 


\begin{abstract}
Human neuroplasticity of multisensory integration has been studied mainly in the context of natural or artificial training situations in healthy subjects. However, substance addicts also offer the opportunity to assess the impact of intensive daily multisensory interactions with addiction-related objects on the neural correlates of crossmodal object processing. The present functional magnetic resonance imaging study revealed that smokers show a comparable visuo-haptic integration pattern for both smoking paraphernalia and control objects in the left lateral occipital complex, a region playing a crucial role in crossmodal object recognition. Moreover, the degree of nicotine addiction correlated positively with the magnitude of visuo-haptic integration in the left LOC for smoking-associated but not for control objects. In contrast, in the left LOC non-smokers displayed a visuo-haptic integration pattern for control objects, but not for smoking paraphernalia. This suggests that prolonged smoking-related multisensory experiences in smokers facilitate the merging of visual and haptic inputs in the lateral occipital complex for the respective stimuli. Studying clinical populations who engage in compulsive activities may represent an ecologically valid approach to investigating the neuroplasticity of multisensory integration.
\end{abstract}




\section{Introduction}

Studies of human multisensory neuroplasticity have demonstrated our brain's striking ability to reorganize as a consequence of experience. Evidence of crossmodal plasticity stems both from studies of sensory deprivation and from experiments investigating the impact of intensive training on the convergence of multisensory information. In monkeys, prolonged training of an auditory discrimination task resulted in the activation of the auditory cortex by visual and somatosensory stimuli as well as by movements (Brosch et al., 2005). In human subjects behavioral training of associations between unfamiliar sounds and images induced a recruitment of superior temporal gyrus/sulcus and intraparietal sulcus in cortical audio-visual integration (Naumer et al., 2009). Furthermore, traininginduced reorganization of cortical and subcortical multisensory interactions has been found in musicians (Pantev et al., 2003; Schulz et al., 2003; D'Ausilio et al., 2006; Musacchia et al., 2007; Hyde et al., 2009). Similarly, long-term experience with two-dimensional shapes in the context of a board game led to enhanced visuo-tactile crossmodal plasticity (Saito et al., 2006).

Intensive or long-term training of a particular activity or task may thus alter the functional network of multisensory convergence in the human brain and facilitate multisensory integration in heteromodal brain regions. So far the basis of multisensory integration plasticity has been studied mainly in healthy or in sensorydeprived (i.e. blind or deaf) individuals (Finney et al., 2001; Bavelier and Neville, 2002; Goldreich and Kanics, 2003; Wittenberg et al., 2004; Bavelier et al., 2006; Hötting and Röder, 2009; Merabet and Pascual-Leone, 2010). Some patient groups, such as substance addicts, also interact excessively and on a long-term basis with substance-related objects resulting in a specific multisensory expertise. Multisensory integration in substance addicts thus provides an opportunity for elucidating the impact of perceptual expertise on crossmodal object processing. In the present study we used functional magnetic resonance imaging (fMRI) to examine whether experience with smoking-related objects changed visuo-haptic integration of smoking paraphernalia in smokers compared to non-smokers. We expected visuohaptic integration effects in the lateral occipital complex, a core region for crossmodal object recognition (Amedi et al., 2001; Amedi et al., 2002; Pietrini et al., 2004; Amedi et al., 2005; Amedi et al., 2007; Stilla and Sathian, 2008; Kim and James, 2009; Lacey et al., 2009; Tal and Amedi, 2009). LOC should be similarly engaged in visuo-haptic integration of everyday control objects in all participants. In contrast, we hypothesized that only smokers but not non-smokers would exhibit a visuo-haptic integration pattern for smoking paraphernalia and that the magnitude of multisensory integration in LOC for smoking-related but not control stimuli would be correlated with the individual degree of nicotine dependence. 


\section{Materials and Methods}

\section{Subjects}

All participants were right-handed, had normal or corrected-to-normal vision and were students or university academic staff. The smokers $(n=15$, mean age 28.3 years $(\mathrm{SD}=3.7), 7$ men and 8 women) were instructed to smoke according to their usual habits but to abstain from smoking $30 \mathrm{~min}$. before the fMRI recordings in order to reduce the confounding influence of nicotine on the blood oxygen level-dependent (BOLD) signal. Their individual level of nicotine dependence was evaluated with the Fagerström Test for Nicotine Dependence [FTND; (Bleich et al., 2002)] after the fMRI sessions. The mean FTND score amounted to $2.8(\mathrm{SD}=2.4$; FTND scores can vary from 0 to 10$)$. We recruited light, medium and heavy smokers to cover the whole spectrum of smoking behavior. The non-smokers $(n=15$, mean age 27 years $(\mathrm{SD}=5.01), 6$ men and 9 women) claimed that they had either never smoked or had smoked a very limited number of cigarettes during their lifetime. Written informed consent was obtained after giving the subjects a complete description of the study.

\section{Experimental design}

Stimuli were four common everyday objects (a pen, a crème box, a small video cassette, an audio cassette; control objects condition) and four smoking-related objects (a cigarette, an ashtray, a normal cigarette box, a longer cigarette box; smoking paraphernalia condition) and gray-scaled pictures of these objects. The objects in the two categories were matched for size and weight and, as far as possible, for roughness of their surface.

Before the fMRI sessions participants performed a haptic recognition task with the experimental objects while lying in the inactive MR scanner. The stimuli were presented to the right subjects' hand by an experimenter in a similar way as in the following fMRI sessions. For each stimulus the participants had 2 seconds to recognize it with eyes closed. They were instructed to identify the object by pronouncing its name (e.g. "a cigarette", "a pen", etc.). The answers were immediately classified as correct or incorrect and written down by the experimenter. If the participant could not recognize a stimulus, it was named to him/her by the experimenter so that in the end all participants were sufficiently familiar with all objects.

During the fMRI recordings, participants actively touched the objects and/or viewed the images. Using a block design, the "smoking paraphernalia" and "control objects" conditions were presented either in the visual modality, in the haptic modality or in both modalities simultaneously. Closing and reopening of the participant's grasping hand was cued by color changes of a centrally presented fixation cross which was present in all conditions. The participants had to follow the color change instructions irrespective of the experimental condition (i.e., also in the visual condition without haptic input). Thus, we created comparable motor components of all three conditions. Haptic stimuli were presented to the participant's hand by an experimenter who received instructions about the on- and offset of stimulation via headphones. Additionally, there was a motor control condition during which participants merely closed and reopened their hand 
following the color changes of the fixation cross without receiving any additional sensory stimulation. This resulted in 7 experimental conditions: visual control (VC), haptic control (HC), visuo-haptic control (VHC), visual smoke (VS), haptic smoke (HS), visuo-haptic smoke (VHS) and motor (M).

The participants underwent four functional runs (two with the left and two with the right hand; the assignment of left or right hand to the respective run was pseudorandomized) and one anatomical scan. At the beginning of each run a fixation interval and a dummy block ensured that T1 saturation effects were avoided. Each experimental condition was repeated twice in separate blocks within each run, resulting in 14 experimental blocks per functional run. The order of the blocks and the runs was pseudo-randomized. Blocks lasted for 24 seconds and alternated with fixation intervals of equal length. Thus, each of the four runs consisted of 15 blocks (14 experimental +1 dummy) and 16 fixation intervals, in total $31 \times 24=744 \mathrm{~s}$ or $12 \mathrm{~min}$. The entire experiment lasted approximately 55 minutes (4 functional runs $\mathrm{x}$ $12 \mathrm{~min}+1$ anatomical scan x $6.6 \mathrm{~min}$ ).

During each block, the following procedure was repeated four times: 3200 ms of fixation preceded a color change of the fixation cross lasting for $300 \mathrm{~ms}$ which instructed the participants to close their hand. The respective experimental stimulus was then presented for $2000 \mathrm{~ms}$ and another color change of the fixation cross signaled to the participants to reopen their hand. This was followed by another 500 $\mathrm{ms}$ of fixation. In the motor condition (M) no stimulus was presented during the $2000 \mathrm{~ms}$ and the participants viewed the default fixation cross. Thus, four stimuli from the same category were presented in a randomized order within each block $(4 \mathrm{x}$ $6000 \mathrm{~ms}=24 \mathrm{~s}$ per block).

The stimuli were presented with Presentation software (Neurobehavioral Systems, Albany, CA, U.S.A.) running on a PC (Miditower Celeron) at a frame rate of $60 \mathrm{~Hz}$. Images were projected onto a vertical screen positioned inside the scanner with an LCD projector (Sony, VPL PX 20) equipped with a custom-made lens. Participants viewed the screen through a mirror fixed on the head coil. The participants' field of view was 52.5 degrees visual angle (maximum horizontal distance).

The fMRI procedure was performed on a 3-Tesla Magnetom Allegra scanner (Siemens, Erlangen, Germany) at the Brain Imaging Center in Frankfurt am Main. A gradient-recalled echo-planar imaging sequence with the following parameters was used: 33 slices; repetition time (TR) $2000 \mathrm{~ms}$; echo time (TE) 30 ms; field of view $192 \mathrm{~mm}$; in-plane resolution $3 \mathrm{~mm}^{2}$; slice thickness $3 \mathrm{~mm}$; gap thickness $0.3 \mathrm{~mm} ; 372$ volumes per functional run. For the anatomical imaging a magnetization-prepared rapid-acquisition gradient echo sequence (176 slices per slab, TR $2250 \mathrm{~ms}$, TE $2.6 \mathrm{~ms}$, flip angle $9 \mathrm{deg}$, matrix $256 \times 256$ and voxel size $1 \mathrm{~mm}^{3}$ ) was used.

After the fMRI sessions the participants filled out the FTND and two additional questionnaires. The first of the additional tests measured the general experience of the subjects with the experimental stimuli. For each object used in the study, the following question had to be answered: "How often do you encounter this object in your everyday life?", whereby the participants had to choose one of the possible answers "very rarely", "rarely", "often", and "very often". The second questionnaire measured the distribution of the subjects' experience with an object between the sensory modalities. Subjects had to estimate which proportion of their 
experience with a particular object stemmed from the visual, haptic and visuo-haptic modality. Thus, regardless of whether someone had little or much experience with an object, they had to estimate whether their experience was predominantly visual, haptic, or visuo-haptic. For example, a subject who had never touched a cigarette, but had seen it frequently (e.g. in advertisements, with other people, etc.), would estimate $100 \%$ visual, $0 \%$ haptic, and $0 \%$ visuo-haptic experience for cigarettes. A heavy smoker would most probably have predominantly visuo-haptic experience with a cigarette (for example 50\%) and the rest of his experience would be distributed among the unisensory modalities (for example 30\% visual and $20 \%$ haptic). Subjects were informed that the sum of the three modality-specific experience distributions should be $100 \%$.

\section{Statistical analysis}

The behavioral recognition test served to determine whether all subjects were similarly familiar with the experimental stimuli and could recognize them even if there was no visual but only haptic input. A recognition index (RI) indicating the proportion of correctly identified objects $(\mathrm{RI}=0$ when no object was recognized, RI $=1$ when all objects were identified) was computed separately for each participant and object category. The RI was subjected to a repeated-measures ANOVA with the within-factor "Object category" (control objects vs. smoking paraphernalia) and the between-factor "Group" (non-smokers vs. smokers).

The data from the first questionnaire measuring general experience with the experimental stimuli were analyzed in the following way: each of the answer options was scored ("very rarely" =1, "rarely" = 2, "often" $=3$, "very often" $=4$ ) and then the mean score was computed for the respective category. Thus, if for example all of the control objects occurred "very often" in a person's everyday life, the mean general experience of this person for the control category would be 4 . Similarly, for the second questionnaire the individual mean percentage of experience was computed separately for the two object categories and the three sensory modalities. Thus, if a person had for example only visual but no haptic or visuo-haptic experience with smoking objects, this would result in $100 \%$ visual, $0 \%$ haptic and $0 \%$ visuo-haptic experience of this person for the object category "smoking paraphernalia". The general experience scores and modality-specific scores that were acquired with the help of the two questionnaires were subjected to two repeated-measures ANOVAs. The first one used the general experience scores as a dependent variable and had the within-subjects factor "Object category" (control objects vs. smoking paraphernalia) and the between-subjects factor "Group" (nonsmokers vs. smokers), while the second ANOVA used the modality-specific scores as a dependent variable and the same two factors as well as the additional withinsubjects factor "modality" (visual vs. haptic vs. visuo-haptic).

Imaging data were analyzed using the BrainVoyager QX 1.10.4 software package (Brain Innovation, Maastricht, The Netherlands). Preprocessing included the following steps: three-dimensional motion correction, linear-trend removal and temporal high-pass filtering at $0.0054 \mathrm{~Hz}$, and slice-scan-time correction with sinc interpolation. Functional and Talairach-standardized anatomical data were used to build a voxel time course file and were spatially smoothed with a Gaussian filter of 8 $\mathrm{mm}$ full width at half maximum. For each voxel the time course was regressed on a 
set of dummy-coded predictors representing the experimental conditions. To account for the shape and delay of the haemodynamic response, the predictor time courses (box-car functions) were convolved with a gamma function.

First, a whole-brain random-effects general linear model (RFX-GLM) based on the data of all participants was run $(\mathrm{n}=30$ [15 smokers +15 non-smokers]). Then a whole-brain statistical map for the conjunction $\mathbf{V C}>\mathbf{M} \cap \mathbf{H C}>\mathbf{M}$ was computed (RFX of conjunction, $\mathrm{t} \geq 5.88, \mathrm{q}<0.005$, corrected for multiple comparisons using the false discovery rate [FDR]). The clusters resulting from this conjunction were then defined as regions of interest where visual and haptic information from objects equally familiar to both smokers and non-smokers ("control objects" condition) converges. We did not use the VS and HS conditions for the definition of the multisensory integration candidate regions, since smokers and non-smokers differ in their experience with smoking paraphernalia and this would have most probably biased the ROI definition. Brain regions consisting of less than 10 voxels were excluded from further analysis.

The ROIs were tested for multisensory integration using the MAXcriterion, which has been established as a measure of multisensory integration in fMRI and is met when the multisensory response is larger than the maximum of the unimodal responses (Beauchamp, 2005; Naumer et al., 2010b). The MAX-criterion was computed separately for both object categories and groups. Thus, the following ROI-based conjunctions were conducted separately for each group: VHC $>$ VC $\cap$ VHC $>$ HC [MAX-criterion for control objects] and VHS $>$ VS $\cap$ VHS $>$ HS [MAX-criterion for smoking paraphernalia].

As we were interested in the association between smoking behavior and the degree of visuo-haptic integration, we searched within the ROIs for correlations between the degree of meeting the MAX-criterion for the two object categories as measured by the fMRI signal and the individual severity of nicotine addiction as indicated by the smoking questionnaire FTND. For this purpose, the MAX-criterion was quantified by computing the multisensory integration index [MSI; (Meredith and Stein, 1986; Wallace et al., 1996; Stanford et al., 2005)]. The individual MSIs for both the control objects and the smoking paraphernalia conditions were computed for each participant based on the beta weights extracted from the respective ROI:

$$
\boldsymbol{M S I}=\left[\frac{\boldsymbol{V H}-\boldsymbol{M A X}(\boldsymbol{V}, \boldsymbol{H})}{\boldsymbol{M A X}(\boldsymbol{V}, \boldsymbol{H})}\right] * 100
$$

where "VH" is the beta weight for the respective bimodal condition and "MAX (V, $\mathrm{H}$ )" is the beta weight for the unimodal condition with the maximal amplitude. Thus, the MSI values quantify the difference between the multisensory response and the most vigorous unimodal response and reflect the enhancement of unimodal responses due to multisensory stimulation. The MSI values were correlated (Pearson correlation) with the external covariate FTND indicating the individual severity of nicotine addiction and the resulting correlations were tested for significance (twosided). 


\section{Results}

The analysis of the behavioral recognition data demonstrated that there was no main effect of the factor "Object category" and no interaction "Object category x Group" but a significant main effect of the factor "Group" (Figure 1A \& Table 1). Thus, smokers exhibited a better haptic recognition ability across all experimental stimuli in comparison to non-smokers but this effect was not dependent on the object category.

The ANOVA for the general experience score revealed a significant main effect of "Group" and a significant interaction "Object category x Group", indicating that smokers had more general experience than non-smokers which was particularly true for smoking paraphernalia (Figure 1B \& Table 1).
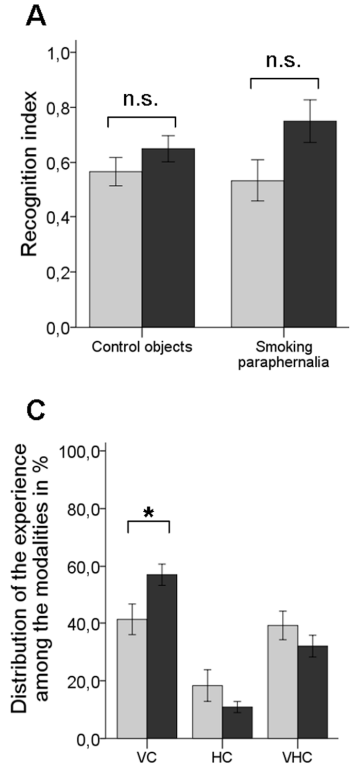
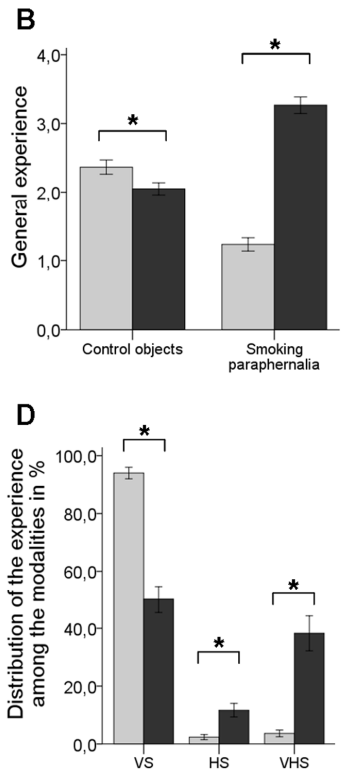

Non-smokers

Smokers

* t-test, $p<0.05$

n.s. t-test non-significant

Figure 1. Results of the behavioral recognition task and general and modality-specific experience questionnaires. A. The recognition index as a function of object category and group; t-tests conducted in addition to the repeated-measures ANOVA presented in Table 1 showed no group differences within the separate object categories. B. General experience as a function of object category and group (see Table 1 for a repeated-measures ANOVA); t-tests showed group differences between the object categories. C \& D. The distribution of the experience with the experimental stimuli among the different sensory modalities is shown separately for the categories "control objects" (C) and "smoking paraphernalia" (D).

The ANOVA for the modality-specific experience percentages resulted in a significant main effect of "Modality" and significant interactions "Modality $\mathrm{x}$ Group", "Object category x Modality" and "Object category x Group x Modality" (Figure 1C \& D as well as Table 1). Additional t-tests were computed in order to understand better the last interaction and the following differences were significant: "VC (smokers) > VC (non-smokers)", "VS (non-smokers) > VS (smokers)", "HS (smokers) > HS (non-smokers)" and "VHS (smokers) > VHS (non-smokers)" (for 
all t-tests $\mathrm{p}<0.05)$, demonstrating that the modality-specific experience distribution differed across groups depending on object category and modality. In particular, smokers reported more haptic and visuo-haptic experiences with smokingparaphernalia than non-smokers, while non-smokers had predominantly visual experiences with these objects.

The whole-brain contrast $\mathbf{V C}>\mathbf{M} \cap \mathbf{H C}>\mathbf{M}$ based on the data of all participants $(n=30)$ showed that visual and haptic information from common objects (control objects condition) converged in the left LOC and superior parietal lobule (SPL) $(\mathrm{q}<0.005$, FDR; see Figure 2 and Table 2).
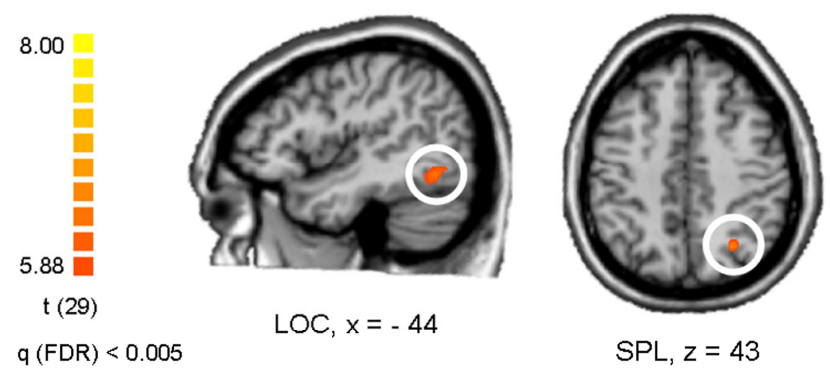

LOC: control objects LOC: smoking paraphernalia
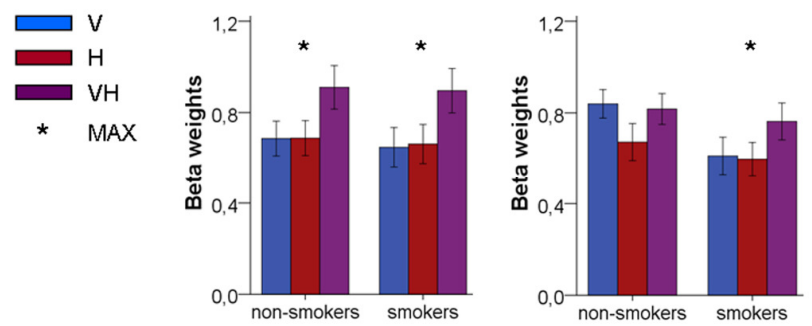

Figure 2. Visuo-haptic integration of control and smoking-related objects in the human brain. In the left LOC and SPL visual and haptic convergence (whole-brain RFX-GLM conjunction VC $>\mathrm{M} \cap \mathrm{HC}>$ M) was observed for control objects in the entire group of participants $(n=30)$. These regions were defined as regions of interest and submitted to further analyses. Beta weights for the different experimental conditions are shown for the left LOC separately for smokers and non-smokers. Error bars indicate the standard error. An asterisk indicates whether the MAX-criterion (VH > MAX $[\mathrm{V}, \mathrm{H}]$ ) was met for the respective group or object category.

Within these regions we applied the MAX-criterion ( $\mathrm{VH}>\mathrm{MAX}[\mathrm{V}, \mathrm{H}])$ separately for the two groups and for each object category. In the left LOC both groups met this criterion for the control objects. However, in the same brain region the MAXcriterion for smoking paraphernalia was met only for smokers (Figure $2 \&$ Tables 3 and 4). The analysis of the left SPL revealed that the MAX-criterion was not met for any of the groups or object categories.

In the next analysis step we tested the two ROIs for correlations between the individual severity of nicotine addiction and the strength of multisensory integration as quantified by MSI values. A significant correlation between the FTND 
and MSI values was found only in the left LOC and only for the multisensory integration of smoking-related but not control objects $(r=0.449, p=0.013$; Figure 3 , Tables 3 and 4). This correlation was still significant even when only the data of the smokers were included $(\mathrm{r}=0.532, \mathrm{p}=0.041)$.

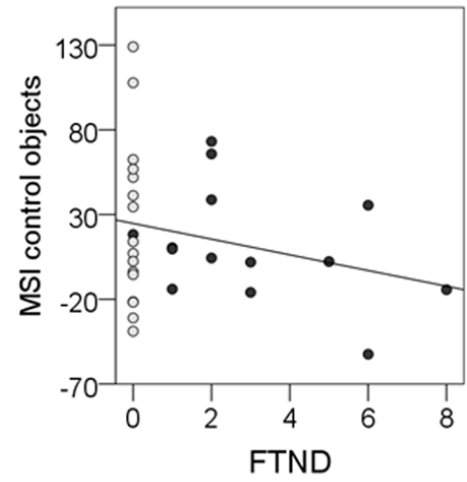

$r=-0.242$, n.s.

O Non-smokers

Smokers

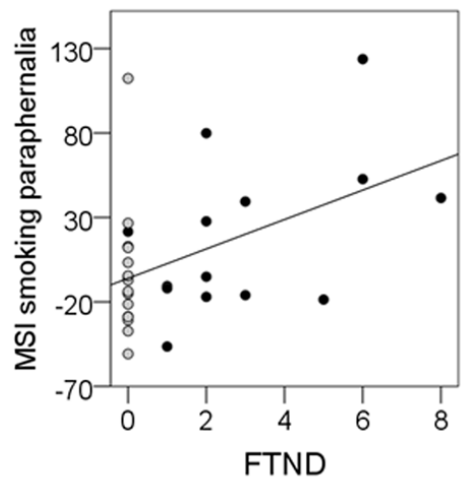

$r=0.449, p=0.013$

O Non-smokers

Smokers

Figure 3. Visuo-haptic integration of smoking-related stimuli in left LOC reflects the degree of nicotine addiction. The scatter plots show the correlations between the individual FTND scores and MSI values for both control objects and smoking paraphernalia in the left LOC which was defined as a ROI in an earlier analysis step.

\section{Discussion}

The present study investigated the impact of smoking-related perceptual experience on the neuroplasticity of multisensory integration. We assessed visuo-haptic integration of everyday objects and smoking paraphernalia in smokers and nonsmokers. Our results confirm previous findings on visuo-haptic object processing. Moreover, they extend the current knowledge on how multisensory integration might be altered by perceptual expertise, and they demonstrate that substance abuse offers a valuable opportunity to study multisensory plasticity in an ecologically valid context.

As expected, the analysis of the questionnaire data revealed that smokers had much more experience with smoking paraphernalia than non-smokers (Figure 1B). This finding can be attributed to the regular usage of smoking-related objects by smokers, who acquire a pronounced perceptual expertise with these stimuli. Furthermore, we found that the smokers' expertise with smoking paraphernalia is to a far greater extent based on haptic and visuo-haptic experience than in non-smokers (Figure 1D). Conversely, non-smokers had mainly visual experiences with smokingrelated objects (Figure 1D), confirming our assumption that non-smokers have little visuo-haptic but a certain visual perceptual expertise for these objects. 
The neural correlates of these bimodal experience differences were reflected by the neuroimaging data. Visuo-haptic convergence ( $\mathrm{VC}>\mathrm{M} \cap \mathrm{HC}>\mathrm{M}$ ) was found for control objects in all participants in the left LOC and SPL. The convergence of visual and haptic information in the SPL is consistent with reports suggesting the SPL or the posterior parietal cortex as brain regions where visual and tactile information converge (Makin et al., 2008; Nakashita et al., 2008). However, the MAX criterion was not met in the left SPL for any of the groups or object categories, i.e., bimodal stimulation did not lead to higher fMRI activations in this region compared to the unimodal conditions. This looks at first surprising given the well-known role of the parietal cortex in multisensory integration (Nakashita et al., 2008). However, as our task was associated rather with shape than with motion perception, the finding that visuo-haptic integration engages predominantly the left LOC, a crossmodal object recognition region, but not the SPL, which is a part of the dorsal visual stream, looks appropriate.

The LOC forms part of the ventral visual pathway and is known to respond preferentially to shape or intact objects as compared to texture patterns or scrambled objects (Malach et al., 1995). Moreover, a part of the LOC termed the lateral occipital tactile-visual region (LOtv) is activated during both visual and tactile object recognition (Amedi et al., 2001; Amedi et al., 2002; James et al., 2002; Pietrini et al., 2004; Stilla and Sathian, 2008; Tal and Amedi, 2009), see (Amedi et al., 2005; Beauchamp, 2005; Lacey et al., 2009) for detailed reviews. Based on this evidence, it has been suggested that the LOC is an object-selective region involved in modality-independent processing of geometric shape (Lacey et al., 2009). Furthermore, visual and haptic processing have been demonstrated to influence each other in the LOC resulting in higher fMRI responses to bimodal than unimodal stimulation (Kim and James, 2009; Naumer et al., 2010a). This enhancement of haemodynamic responses due to bimodal stimulation can be quantified by the MAX criterion and the MSI scores.

Interestingly, the visuo-haptic integration pattern in the left LOC covaried with object category, group membership and degree of nicotine addiction. While both smokers and non-smokers met the MAX criterion in the left LOC for control objects, only smokers met it for smoking paraphernalia. Moreover, the degree of meeting the MAX criterion as quantified by MSI values correlated positively with the severity of nicotine addiction as measured by FTND scores. More importantly, this was true only for smoking paraphernalia but not for control objects, which further corroborates our assumption that smoking-related experience modulates the cortical integration of vision and haptics for the respective object category.

Based on these results, we propose that in the course of nicotine addiction, the merging of visual and haptic information related to smoking paraphernalia progressively engages the left LOC. Smokers are confronted daily with smokingassociated visual and haptic inputs and rely on their successful integration to recognize the respective objects, interact with them and initiate a smoking action. Such intensive perceptual experience over extended periods of time may have facilitated the development of optimized visuo-haptic integration mechanisms for these objects in the lateral occipital cortex. Essentially, this interpretation is consistent with reports of training-induced effects on human neural plasticity which have demonstrated that shape-related activity in the LOC can be altered by intensive experience (Op de Beeck et al., 2006; Jiang et al., 2007). Similarly, crossmodal 
transfer of object shape information triggered by training has been shown to involve the LOC (Amedi et al., 2007). Moreover, the benefits of multisensory learning for perceptual and behavioral performance have recently been emphasized (Seitz et al., 2006; Seitz et al., 2007; Shams and Seitz, 2008). Crossmodal interactions seem to be most susceptible to environmental influences early in life (Wallace and Stein, 2007; Batterson et al., 2008), but multisensory integration patterns can be modified or even acquired in adults (Gonzalo et al., 2000; Schulz et al., 2003; Tanabe et al., 2005; Naumer et al., 2009), whereby familiarity with particular objects can facilitate their crossmodal perception (Bushnell and Baxt, 1999; Lacey and Campbell, 2006). These findings support the assumption that visuo-haptic integration of smoking-related objects may indeed be obtained on the basis of the smokers' experience with smoking paraphernalia.

Essentially, smokers did not differ from non-smokers in their recognition ability for haptically presented smoking paraphernalia (see Figure 1A). Thus, while non-smokers could recognize smoking-related objects as well as smokers when only haptic input was present, only smokers exhibited a neural visuo-haptic integration pattern in their left LOC. This shows that insufficient recognition ability of nonsmokers cannot account for their lack of multisensory integration of smoking paraphernalia as quantified by the fMRI signal. Therefore it seems more likely that the inferior visuo-haptic integration of smoking objects in non-smokers is attributable to their lesser multisensory smoking-related experience.

One of the limitations of the current study is the smokers' relatively moderate level of nicotine dependence. The FTND score can vary between 0 and 10 and the mean FTND score of the smokers in our experiment was 2.8, indicating that the majority of the participants were light to medium smokers. Possibly, if the mean severity of nicotine addiction was higher, the observed effects would have been even stronger, for example with regard to the correlations between the FTND and the MSI scores.

Future research might investigate visuo-haptic integration of smoking paraphernalia in ex-smokers. Whether their cortical multisensory integration pattern for smoking-related objects is more similar to the one of current smokers or the one of non-smokers would provide important information concerning the question of how multisensory integration is formed, maintained and developed across the life span. Thus, the observation that "training" a bimodal behavior leads to multisensory integration could be extended by studying the effects of abstaining from a previously trained behavior: would crossmodal integration decline or stay the same?

The current results may be relevant not only in the context of crossmodal plasticity, but also for understanding addiction. Our study offers the possibility to transfer knowledge from basic research on multisensory integration to the clinical domain and to contribute to the understanding of the etiology, diagnostics and treatment of addiction. Similar experience-dependent plasticity in nicotine addiction has already been demonstrated for the sensorimotor domain, where we found relationships between the activation of sensorimotor brain regions by visual smoking-associated cues, automatized smoking-related motor schemata and the severity of nicotine dependence (Yalachkov et al., 2009), see (Yalachkov et al., 2010) for a review. Visuo-haptic integration in smokers emphasizes the relevance of experience-based neural plasticity for the understanding of addiction. Since multisensory integration usually results in enhanced detection rates and orienting 
responses (Stein and Meredith, 1993; Naumer and Kaiser, 2010), efficient multisensory integration of drug-related cues obtained by everyday experience with these objects may provide them with particular salience, thus reducing the threshold for an overt response towards them. Thus, future research should address the question whether robust visuo-haptic integration of smoking paraphernalia is not only the consequence of prolonged experience, but may in some cases also contribute to the initiation of smoking actions and thus to relapses in individuals who are trying to abstain from smoking. Moreover, integrating visual and haptic smoking cues appears to be a perceptual function which is modified by smoking behavior in smokers and studying it may be an interesting approach towards establishing a reliable biomarker of addiction.

The present paper demonstrates that while both smokers and non-smokers show visuohaptic integration for control objects in the left LOC, only smokers exhibit a similar fMRI signal pattern for smoking paraphernalia. Furthermore, this effect is correlated positively with the severity of their nicotine addiction. These findings emphasize the value of studying patient populations for understanding the mechanisms of multisensory integration plasticity. Clinical subjects such as substance addicts offer a unique possibility to study multisensory integration changes which are established as a consequence of intensive engagement in addictive activities and long-term perceptual experience with the respective paraphernalia.

Acknowledgments: We thank Christoph Bledowski for helpful discussions. 


\section{Tables}

Table 1. Three repeated-measures ANOVAs were computed with the dependent variables "recognition index" (within-factor "Object category": control objects vs. smoking paraphernalia; between-factor "Group": non-smokers vs. smokers), "general experience score" (within-factor "Object category": control objects vs. smoking paraphernalia; between-factor "Group" non-smokers vs. smokers) and "modalityspecific percentage of experience" (within-factor "Object category": control objects vs. smoking paraphernalia; within factor "Modality" visual vs. haptic vs. visuo-haptic; between-factor "Group": nonsmokers vs. smokers).

\begin{tabular}{|c|c|c|c|c|}
\hline & Source of variance & Type III sum of squares & F-value & p-value \\
\hline 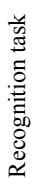 & $\begin{array}{l}\text { Object category } \\
\text { Group } \\
\text { Object category } \times \text { Group }\end{array}$ & $\begin{array}{l}0.017 \\
0.338 \\
0.067\end{array}$ & $\begin{array}{r}0.222 \\
6.69 \\
0.887\end{array}$ & $\begin{array}{l}\text { n.s. } \\
0.015 \\
\text { n.s. }\end{array}$ \\
\hline 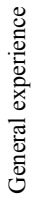 & $\begin{array}{l}\text { Object category } \\
\text { Group } \\
\text { Object category } \times \text { Group }\end{array}$ & $\begin{array}{r}0.026 \\
11.051 \\
20.709\end{array}$ & $\begin{array}{c}0.189 \\
61.68 \\
150.288\end{array}$ & $\begin{aligned} & \text { n.s. } \\
< & 0.001 \\
< & 0.001\end{aligned}$ \\
\hline 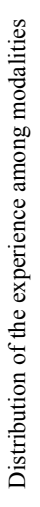 & $\begin{array}{l}\text { Object category } \\
\text { Modality } \\
\text { Group } \\
\text { Object category x Modality } \\
\text { Object category } \times \text { Group } \\
\text { Modality x Group } \\
\text { Object category x Modality x Group }\end{array}$ & $\begin{array}{c}1.8 \\
76621.3 \\
1.8 \\
11931.008 \\
1.8 \\
5802.008 \\
20980.633\end{array}$ & $\begin{array}{c}1 \\
85.728 \\
1 \\
24.874 \\
1 \\
6.492 \\
43.74\end{array}$ & $\begin{array}{l}\text { n.s. } \\
<0.001 \\
\text { n.s. } \\
<0.001 \\
\text { n.s. } \\
0.021 \\
<0.001\end{array}$ \\
\hline
\end{tabular}

Table 2. Brain regions where the whole-brain GLM-RFX conjunction $\mathrm{VC}>\mathrm{M} \cap \mathrm{HC}>\mathrm{M}$ was met $[\mathrm{n}=$ 30 ; $\mathrm{df}=29 ; \mathrm{q}<0.005$, FDR; voxel size $=1 \times 1 \times 1 \mathrm{~mm}]$

\begin{tabular}{|c|c|c|c|c|c|c|}
\hline \multirow[b]{2}{*}{ Region } & \multicolumn{3}{|c|}{$\begin{array}{l}\text { Talairach } \\
\text { coordinates }\end{array}$} & \multirow[b]{2}{*}{$\mathrm{Nr}$ of voxels } & \multicolumn{2}{|c|}{ RFX of Conjunction } \\
\hline & $\mathrm{x}$ & $\mathrm{y}$ & $\mathrm{z}$ & & Average t-value & Average p-value \\
\hline left lateral occipital cortex & -45 & -58 & -9 & 477 & 6.16 & 0.000001 \\
\hline left superior parietal lobule & -26 & -60 & 43 & 594 & 6.17 & 0.000001 \\
\hline
\end{tabular}


Table 3. ROI-based analysis for non-smokers $(\mathrm{n}=15$; $\mathrm{df}=14)$ for the two object categories. $[\mathrm{MAX}] \sqrt{ }=$ MAX-criterion is met in this ROI $[\mathrm{MAX}] \mathrm{X}=\mathrm{MAX}$-criterion is not met in this ROI

\begin{tabular}{|c|c|c|c|c|c|c|}
\hline Region & MAX control & $\mathrm{VHC}>\mathrm{VC}$ & $\mathrm{VHC}>\mathrm{HC}$ & MAX smoking & VHS $>$ VS & VHS $>$ HS \\
\hline $\begin{array}{l}\text { left lateral occipital } \\
\text { cortex }\end{array}$ & $\sqrt{ }$ & $\begin{array}{l}\mathrm{t}=2.945 \\
\mathrm{p}=0.011\end{array}$ & $\begin{array}{l}\mathrm{t}=3.098 \\
\mathrm{p}=0.008\end{array}$ & $\times$ & $\begin{array}{c}\mathrm{t}=-0.515 \\
\text { n.s. }\end{array}$ & $\begin{array}{c}\mathrm{t}=1.898, \\
\text { n.s. }\end{array}$ \\
\hline $\begin{array}{l}\text { left superior parietal } \\
\text { lobule }\end{array}$ & $\times$ & $\begin{array}{l}\mathrm{t}=8.572 \\
\mathrm{p}=0.000\end{array}$ & $\begin{array}{c}\mathrm{t}=1.768 \\
\text { n.s. }\end{array}$ & $\times$ & $\begin{array}{c}\mathrm{t}=-0.434 \\
\text { n.s. }\end{array}$ & $\begin{aligned} \mathrm{t}= & 1.130 \\
& \text { n.s. }\end{aligned}$ \\
\hline
\end{tabular}

Table 4. ROI-based analysis for smokers $(\mathrm{n}=15 ; \mathrm{df}=14)$ for the two object categories. $[\mathrm{MAX}] \sqrt{ }=$ MAX-criterion is met in this ROI $[\mathrm{MAX}] \mathrm{X}=\mathrm{MAX}$-criterion is not met in this ROI

\begin{tabular}{|c|c|c|c|c|c|c|c|c|}
\hline Region & $\begin{array}{l}\text { MAX } \\
\text { control }\end{array}$ & $\mathrm{VHC}>\mathrm{VC}$ & $\mathrm{VHC}>\mathrm{HC}$ & $\begin{array}{c}\text { MAX } \\
\text { smoking }\end{array}$ & $\mathrm{VHS}>\mathrm{VS}$ & VHS $>$ HS & $\begin{array}{l}\text { Correlation } \\
\text { FTND } \\
\text { \& MSI } \\
\text { control } \\
\text { objects }\end{array}$ & $\begin{array}{c}\text { Correlation } \\
\text { FTND } \\
\& \text { MSI } \\
\text { smoking } \\
\text { paraphernalia }\end{array}$ \\
\hline $\begin{array}{l}\text { left lateral } \\
\text { occipital } \\
\text { cortex }\end{array}$ & $\sqrt{ }$ & $\begin{array}{l}\mathrm{t}=3.276 \\
\mathrm{p}=0.006\end{array}$ & $\begin{array}{l}\mathrm{t}=3.226 \\
\mathrm{p}=0.006\end{array}$ & $\sqrt{ }$ & $\begin{array}{l}\mathrm{t}=2.213 \\
\mathrm{p}=0.044\end{array}$ & $\begin{array}{l}\mathrm{t}=2.360 \\
\mathrm{p}=0.033\end{array}$ & n.s. & $\begin{array}{l}r=0.449 \\
p=0.013\end{array}$ \\
\hline $\begin{array}{l}\text { left } \\
\text { superior } \\
\text { parietal } \\
\text { lobule }\end{array}$ & $\times$ & $\begin{array}{c}\mathrm{t}=1.719, \\
\text { n.s. }\end{array}$ & $\begin{array}{c}\mathrm{t}=2.624 \\
\mathrm{p}=0.02\end{array}$ & $\times$ & $\begin{array}{c}\mathrm{t}=-0.579 \\
\text { n.s. }\end{array}$ & $\begin{array}{c}\mathrm{t}=0.573 \\
\text { n.s. }\end{array}$ & n.s & n.s \\
\hline
\end{tabular}



Chapter 6

General discussion 


\section{General discussion}

Addiction is a chronic disease with a strong impact on public health. Biology, psychology and medicine have tried to reduce addiction-related harms and costs by understanding how and why addiction develops, which individuals are particularly at risk and which are the best prevention strategies and treatments. Among the different scientific fields dealing with addiction, neuroscience has substantially contributed to understanding the disease mechanisms and to the development of efficient treatments. Moreover, its findings have a much deeper impact than expected: while the majority of studies have demonstrated that chronic drug use results in longlasting alterations in brain structure, function, and connectivity, it is now particularly hard to define addiction as a problem of "free will" or to view it solely as a psychological problem (Volkow and Li, 2004; Hyman, 2007). Therefore, studying the neurobiological underpinnings of addiction may not only elucidate its underlying mechanisms but also change prejudices against addicts.

The most vigorously researched topic in addiction neuroscience is how drugs of abuse interact with processes of the healthy brain like learning, memory and cognitive control to eventually establish substance dependence. It is assumed

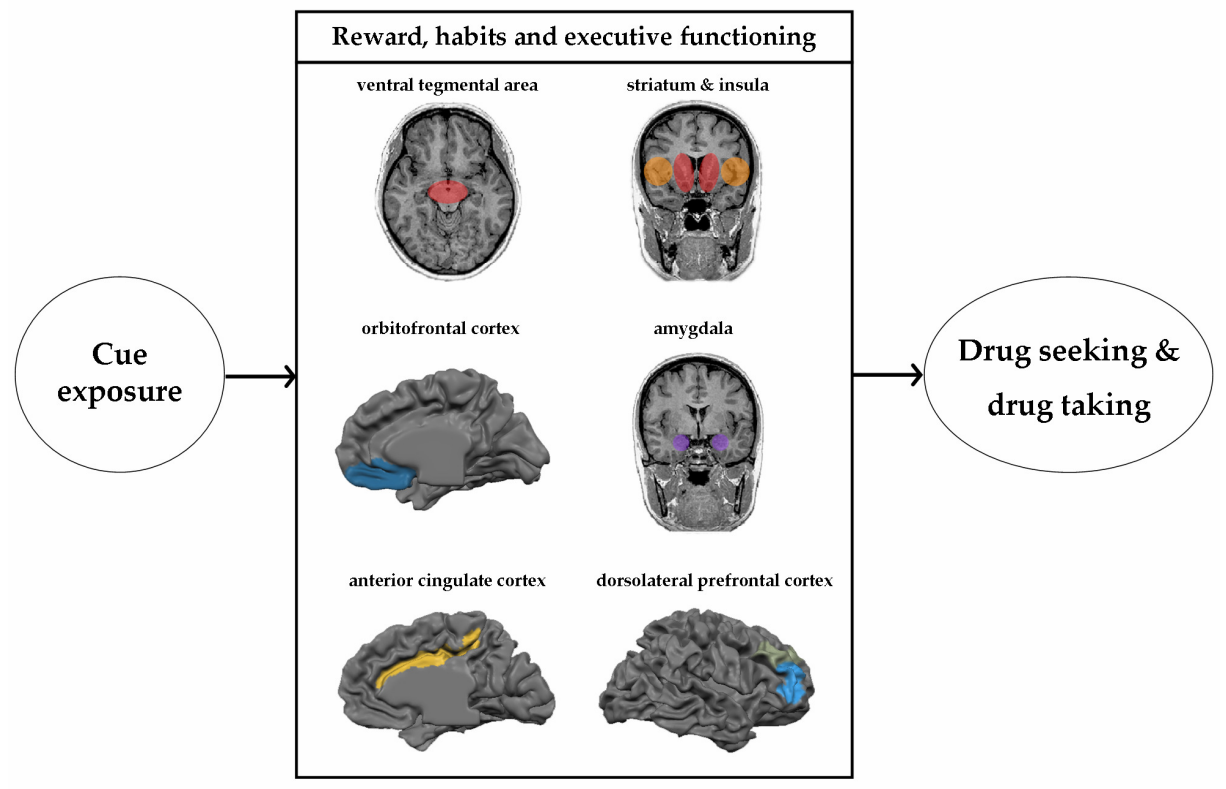

Figure 1. Brain systems involved in neural cue reactivity and relapse according to the current knowledge. This simplified version of the current neuroscientific models of addiction shows the brain regions which are associated with the different mechanisms of addiction such as reward motivation (ventral tegmental area, ventral striatum, orbitofrontal cortex, amygdala, insula), habits (dorsal striatum) and impaired executive functioning (anterior cingulate, dorsolateral prefrontal cortex and insula). The hippocampus and thalamus are not depicted due to space limitations; however, their role in addiction is indisputable. For more information on the well-established neuroscientific concepts of addiction see Chapter 1. 
that reward-related processes are hijacked by chronic drug abuse and lead to prominent changes in the reward and habit systems of the brain. These neural alterations, combined with impaired behavioral inhibition functions, leave the individual highly susceptible to the incentive values and action associations of conditioned drug cues (Volkow et al., 2004; Hyman, 2005; Baler and Volkow, 2006; Hyman et al., 2006; Garavan and Hester, 2007; Goldstein et al., 2009; Koob and Volkow, 2010). Thus, in a complex interaction the reward- and habit-associated brain networks are activated by drug cues (e.g. paraphernalia, people, context, internal states, etc.) and drive the individual towards drug seeking, while the control over drug use behavior fails due to the impaired executive functions (see Figure 1 for a schematic model of the brain systems involved in cue reactivity and relapse).

The present thesis sought to complement the current state of knowledge by exploring how sensorimotor and multisensory aspects of addiction are involved in these processes. After providing a brief introduction to the general neuroscience of addiction in Chapter 1, I reviewed the literature on sensory and motor processes in the context of addiction in Chapter 2. While intensive and regular involvement in activities with particular objects results in deeply embedded action schemata which contain the corresponding motor knowledge that is quickly accessible and can easily be triggered (Tiffany, 1990; Johnson-Frey, 2004; Calvo-Merino et al., 2005; CalvoMerino et al., 2006; Lewis, 2006; Weisberg et al., 2007), animal experiments have suggested that drugs of abuse like cocaine facilitate brain circuits responsible for developing such motor programs (Willuhn et al., 2003; Willuhn and Steiner, 2006, 2009). This implies that in the human addict the "training" effect of using drug paraphernalia several times per day would be complemented by the direct neuropharmacological effects of drugs of abuse and would eventually lead to even more strongly represented drug-associated motor schemata. Furthermore, it has been demonstrated that sensory cues acquire motivational values dependent on their reward history. This process is reflected not only by reward but also by sensory and perceptual systems (Serences, 2008). Again, animal research points to the facilitating impact of cocaine on the neural processing of sensory cues (Devonshire et al., 2004; Devonshire et al., 2007). Thus, in the context of human drug use, cocaine might enhance not only the development of motor schemata but also the sensory processing of drug cues such as the used paraphernalia resulting in salient brain representations of these stimuli. Similar findings have been reported for nicotine. Nicotine administration facilitates the corticospinal excitability leading to increased motor-evoked potentials, and the stimulation of nicotinic acetylcholine receptors located in different sensory cortices contributes to the enhancement of sensory responses (Metherate, 2004; Swayne et al., 2009). Interestingly, a large number of studies has provided evidence that drug cues activate preferentially not only reward- or habit-related but also sensory, sensorimotor and motor brain regions (Yalachkov et al., 2010). These activations could reflect the enhanced sensory and semantic representations of drug paraphernalia as well as the persistent drug-related motor schemata. A further observation that stresses the relevance of sensory and motor processes for addiction is that activations of the respective brain regions in response to drug-associated cues can predict relapse and correlate with craving, severity of dependence and with automatized behavioral reactions towards drugrelated stimuli (Yalachkov et al., 2010). 
Chapter 3 dealt with the question whether visual smoking-related cues can activate the neural representations of the corresponding drug taking skills and action knowledge in smokers. Using fMRI and a behavioral orientation affordance paradigm, we revealed that smokers exhibit higher BOLD signals than non-smokers when viewing smoking-related but not control images. These group differences in fMRI activations were located not only in brain regions associated with reward, habits and decision-making (striatum, dorsolateral prefrontal cortex, insula, etc.), but also in regions implicated in the encoding of action knowledge and tool use skills (bilateral premotor cortex, left superior parietal lobule, and right lateral cerebellum). A behavioral affordance reaction-time task indicated that smokers, but not nonsmokers, showed an automatized responsiveness to smoking paraphernalia similar to everyday objects. Moreover, smokers showed strong intercorrelations between fMRI activations in tool use-related brain regions, behavioral responsiveness to smokingrelated cues, and severity of nicotine dependence. Apparently, the brain representations of smoking-related motor schemata and action skills can be activated by visual cues and the effect is reflected not only on the neural but also on the behavioral level. Even more importantly, the correlation of these activations with the individual degree of nicotine dependence suggests that fMRI and behavioral measurements of automatized action schemata in smoking can be used for the assessment of the patient's severity of addiction as well as the risk of relapse and therapy success.

However, attempts to develop reliable and valid biomarkers of addiction by measuring fMRI activations have proved rather difficult. Since different neuroimaging studies have not identified the same brain regions, it has been proposed that brain responses to drug stimuli can be modulated by additional factors which remain to be elucidated (Wilson et al., 2004). The study described in Chapter 4 is an attempt to reveal the impact of one of those factors by investigating how smoking cue reactivity is modulated by the sensory modality in which the stimuli occur. Employing fMRI, the Fagerström Test for Nicotine Dependence (Bleich et al., 2002) and a simple behavioral task, we addressed this question while smokers touched smoking paraphernalia and control objects or viewed the gray-scaled images of these stimuli. Our findings suggest that while the dorsal striatum, dorsal premotor cortex (PMd) and DLPFC are more responsive to haptic than to visual smoking cues, the cerebellum prefers visual over haptic smoking-related stimuli. A possible explanation for these differential neural cue reactivity patterns has been outlined by a number of studies which have shown that cue reactivity co-varies with the perceived availability of the substance (Carter and Tiffany, 2001; Bailey et al., 2010). Moreover, a recent review has proposed that participants who exhibit a more pronounced perceived availability of the drug, also show stronger neural cue reactivity in the DLPFC (Wilson et al., 2004). Thus, haptic cues are most probably experienced by smokers as a more powerful predictor and trigger of smoking behavior than visual information, resulting in the more prominent engagement of the DLPFC. Furthermore, haptic perception is more tightly linked to action than visual perception and therefore more pronounced haemodynamic responses of the dorsal striatum and PMd (brain regions representing action schemata and habits) to tactile smoking stimuli are not surprising. Finally, cognitive neuroscience research has linked the cerebellum to action schemata and tool use. However, in the context of addiction it has been identified also as an important structure involved in craving 
when visual drug cues are presented (Grant et al., 1996), which fits well to its greater responsiveness to visual than haptic smoking stimuli in our experiment. One implication of these findings for the development of reliable neurobiological markers of addiction, effective cue-exposure therapies and ecologically valid experimental designs is that the sensory modality of drug cues should be more strongly considered in addiction research and clinical practice. Haptic cues could be used to evoke more pronounced cue reactivity especially in the dorsal striatum, which seemed to respond in smokers but not in non-smokers preferentially to haptically as compared to visually presented smoking paraphernalia. Of course, an intriguing question is why visual smoking cues induced neural cue reactivity in the dorsal striatum and the premotor cortex in the study reported in Chapter 3 but not in the study described in Chapter 4. As outlined in Chapter 4, the reason for this difference might be the kind of stimuli that were used in the two studies. While in the first study (Chapter 3) images were presented depicting people who were smoking and thus the participants saw not only smoking-related objects but also faces, hands and the respective environment, in the second study (Chapter 4) only images of smoking-paraphernalia were used. I suggest that the visual stimuli in the second study may not have been salient enough to surpass the cue reactivity threshold in the dorsal striatum and premotor cortex. On the contrary, haptic cues seemed particularly effective in the same experiment and were able to induce strong fMRI responses in those brain regions. In summary, our finding that the sensory modality of the drug stimuli affects neural cue reactivity has important implications for the neuroscience of addiction, since the dorsal striatum, PMd, DLPFC and cerebellum play a prominent role in mediating drug-related habits, rewardassociated motivation and decision-making.

Chapter 5 pursued the question of how drug-related perceptual experience alters the multisensory integration efficiency on the neural level. Since smokers rely in their everyday life on integrating efficiently visual and haptic sensory information about the smoking cues in order to successfully initiate and carry out the corresponding action sequences like grasping the cigarettes and the lighter, lifting them up, using them, etc., they should possess a superior multisensory integration expertise for smoking paraphernalia compared to non-smokers. This question is of interest to both multisensory and addiction research. Studying subjects who engage regularly in compulsive activities such as drug consumption offers a new opportunity to investigate the neuroplasticity of multisensory integration. On the other hand, multisensory integration, unlike higher cognitive and motivational functions, is a rather basic perceptual process which may be less susceptible to bias and thus be more suitable as a biological marker of addiction. Indeed, we demonstrated that in the left LOC, a brain region crucial for crossmodal object recognition, smokers show enhanced visuo-haptic integration for smoking paraphernalia but not for control objects in comparison to non-smokers and that this effect is dependent on the severity of nicotine addiction.

However, there are some questions which remain unanswered by the present thesis and require further investigation. Can the effects reported in Chapter 3 be replicated with other neuroimaging techniques? fMRI has a higher spatial resolution than electro- (EEG) or magnetoencephalography (MEG), which is of great value when localizing the neural cue reactivity differences between smokers and control subjects. Nevertheless, methods like EEG or MEG, which have a better 
temporal resolution than fMRI, could contribute valuable information about the temporal characteristics of the automatized action schemata in smokers and nonsmokers. If these are rather basic neural processes triggered automatically by smoking cues, this should be visible in early sensorimotor activity changes.

Another technique, which could be successfully employed to extend the knowledge about sensorimotor and multisensory processes in nicotine addiction, is transcranial magnetic stimulation (TMS). Since in a typical TMS study the experimentator temporarily stimulates or blocks the activity of cortical regions applying electromagnetic pulses, this would be particularly helpful for developing a study design in which either the automatized reactions towards smoking cues are inhibited or the visuo-haptic integration of the same cues is disrupted. The latter application of TMS would represent an interesting approach to test the relevance of multisensory integration for drug seeking behavior: if successful visuo-haptic integration of smoking paraphernalia is necessary for triggering drug use behavior, a temporary disruption of the neural activity in the underlying regions should lead to diminished cue reactivity.

The nature of the visuo-haptic integration processes in smokers could be studied more precisely in the future. While multisensory integration can be modified or even acquired by training, we cannot clearly separate the influence of multisensory integration expertise in smokers from the reinforcing effects of the drugs which have been regularly paired with visual and haptic cues. Due to the direct pharmacological effects of nicotine, dopamine transmission in the mesocorticolimbic reward system is elevated during acute consumption. However, there are dopaminergic projections also to the occipital and temporal lobes of the mammalian brain (Takada and Hattori, 1987; Mansour and Watson, 2000; Muller and Huston, 2007; Vitay and Hamker, 2007). Since dopamine is a crucial factor for triggering neuroplasticity (Dani et al., 2001; Jones and Bonci, 2005), it is conceivable that dopaminergic bursts during acute nicotine administration additionally facilitate the merging of sensory modalities in the lateral occipital cortex. A promising experimental design to try to disentangle these two factors may include current smokers, who are still exposed to the pharmacological effects of nicotine, and smokers, who no longer smoke cigarettes but use the "electronic cigarette". However, in many of the cases the electronic cigarette devices still deliver nicotine via a vaporized nicotine solution. Therefore, the subjects should be carefully selected and only those should be included who use devices without nicotine.

Studying different populations of smokers seems an interesting approach to reveal how different aspects of nicotine addiction change over the course of the disease and to test whether and how some of the mechanisms of addiction are still active even after prolonged abstinence. Ex-smokers are particularly interesting for this kind of research. They could be compared with current smokers and nonsmokers regarding their performance in smoking-related sensorimotor and multisensory tasks. Furthermore, subjects who are addicted to other kinds of substances should be tested with the same paradigms. Alcohol, for example, has the second greatest impact on public health after nicotine. Excessive and regular drinking is associated with intensive and prolonged sensorimotor and multisensory experience: drinkers rely on integrating visual alcohol-related inputs with haptic information coming from the glass or the bottle in order to represent the 
corresponding object on the neural level, to access the correct semantic information and to initiate the appropriate action sequence. Because drinking even just a normal bottle of beer implies pouring the beer in the glass and lifting up the glass to your mouth at least several times, frequent alcohol consumers might possess wellembedded drinking action schemata, which are easily triggered by conditioned cues. However, it could be difficult picking up the appropriate control group, because everyone drinks water or other non-alcoholic beverages quite often using a similar action schema. Thus, even a control subject could activate pronounced drinking action schemata. The confounding influence of this factor could prove quite difficult to control for. A possible solution of this problem could be related to the specific size and form of glasses for different beverages: many of the glasses used for example for beer consumption are bigger and have to be grasped with the whole hand (power grip) while simple small cups for coffee are often held between the index finger and the thumb (precision grip). The difference between a power and a precision grip has been already utilized in affordance paradigms to measure the behavioral and neural activation of motor schemata (Ellis and Tucker, 2000; Grezes and Decety, 2002) and could therefore be implemented in the context of experiments studying alcohol-specific automatized action knowledge.

All in all, there are several issues, which need to be taken into account when discussing the findings of this $\mathrm{PhD}$ thesis. Future investigations should employ further neuroimaging techniques to replicate the present results and to test for questions that could not be answered by the studies included in this thesis. Different populations of addicts (ex-smokers, other substance abusers) should be recruited to study the alterations of sensorimotor and multisensory mechanisms during the course of addiction and to enhance the external validity of our findings.

In summary, the present thesis complements the current state of knowledge in substance dependence research and serves a better understanding of the underlying mechanisms of addiction. While I fully acknowledge and appreciate the findings reported in the last years and the models of addiction that have been developed on their basis (see Chapter 1 for an overview), I suggest that there are at least two facets of addiction left which have not gained the necessary attention from the research community yet. These facets are the addict's perceptual and actionrelated processes with their respective sensorimotor and multisensory aspects. In Chapters 2-5 I presented evidence that sensory and motor representations of drugassociated objects and actions are reflected on the neural level and are correlated both with the severity of nicotine dependence and with automatized behavior. Importantly, the sensory modality of the drug cues modulates the corresponding neural cue reactivity, which is an essential insight for the neuroscience of addiction. More precisely, haptic cues seem particularly efficient in inducing neural cue reactivity in brain regions involved in different mechanisms of addiction and should therefore be carefully considered when developing biological markers, cue-exposure therapies and experimental designs. Integrating visual and haptic smoking cues appears to be another perceptual function which is modified by experience in smokers and studying it may be an interesting approach towards establishing a reliable biomarker of addiction (see Figure 2 for a summary of the findings of this thesis and how they complement the current state of knowledge).

On the other hand, the observations reported in Chapter 5 indicate that substance abuse populations can be used for a more ecologically valid investigation 
of multisensory integration mechanisms: due to their regular natural experience with drug-related objects addicts possess a strongly pronounced multisensory integration

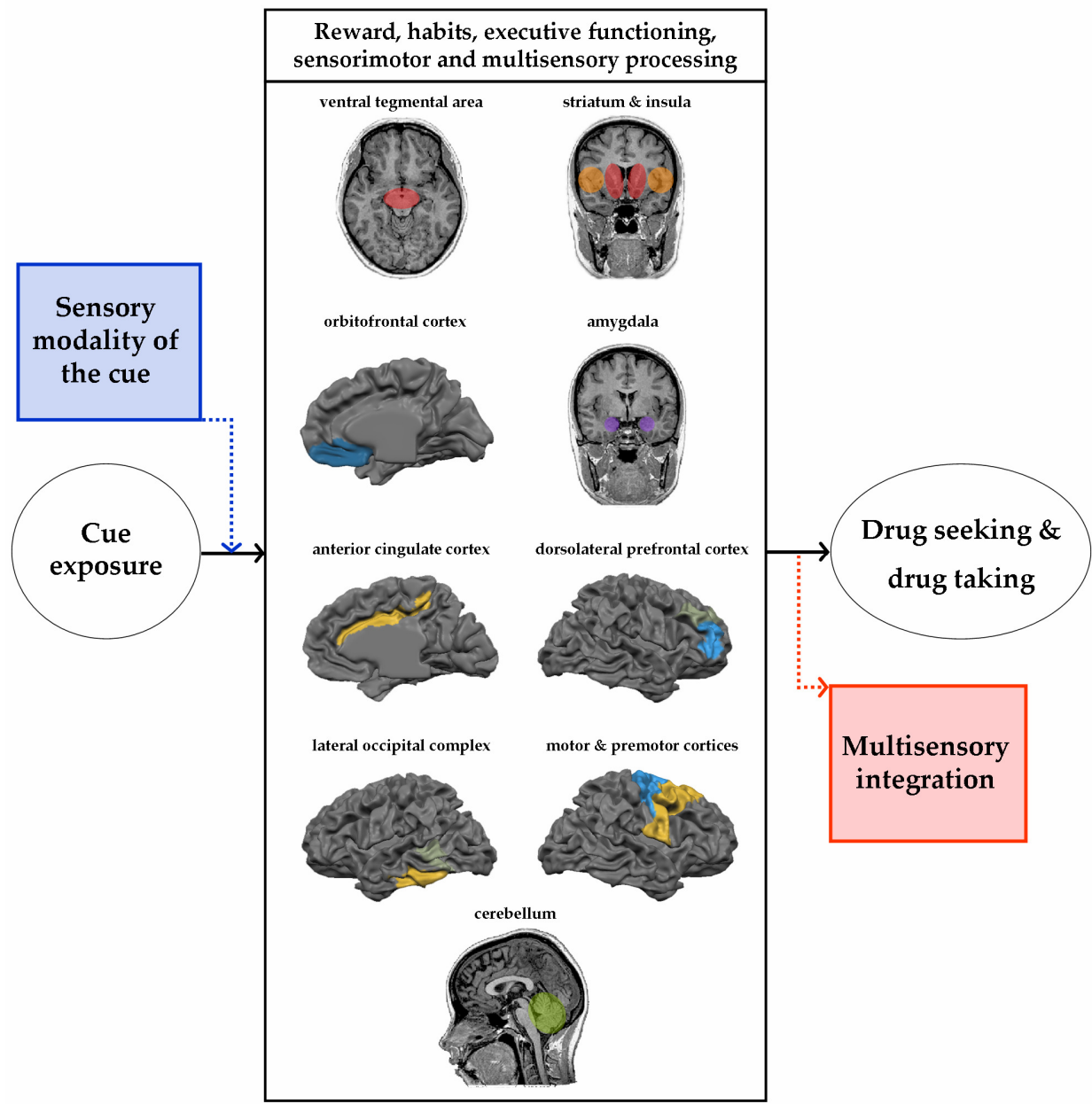

Figure 2. An extended version of the schematic model presented in Figure 1. The most significant contributions of the present thesis complement the current state of knowledge in the neuroscience of addiction. First, our findings provide evidence that the sensory modality in which the drug cues occur modulates neural cue reactivity. Furthermore, the sensorimotor cortices and the cerebellum play clearly an important role in the cue reactivity of the addicted brain, especially for the neural representation of drug-associated habits and automatized action schemata. Finally, the present work demonstrates that the multisensory integration of drug stimuli is modified in crossmodal regions such as the lateral occipital complex due to extensive experience with the corresponding paraphernalia. Thus, not only reward motivation, habits and executive functioning but also sensorimotor and multisensory processes are involved in the mechanisms of addiction. Moreover, the neuroplasticity of perceptual functions such as multisensory integration can be studied in substance addicts.

for these paraphernalia. Their multisensory integration performance can be compared with that of non-users to find out how regular use of particular objects modifies the multisensory perceptual functions on the neural and behavioral level. Finally, the successful combination of multisensory integration and addiction-related 
research demonstrates how cognitive and clinical neuroscience can work together in a way which is highly beneficial for both fields. Thus, more intensive links and cooperations between these two fields of science should be encouraged in order to achieve a better understanding of addiction as well as a more sophisticated approach of fundamental neuroscience to the question of multisensory integration. 

Summary 


\section{Summary}

Addiction is one of the diseases with the highest impact on society as indicated by the estimated addiction-related deaths and emerging costs for the health system. Understanding the underlying mechanisms of this disease has proved to be a rather difficult task but neuroscience has nevertheless made important steps showing that drugs of abuse hijack brain processes underlying learning, memory and cognitive control to establish compulsive drug seeking and drug taking behaviors that are highly resistant to modifications (a brief overview over the established findings in the neuroscience of addiction is given in Chapter 1).

The present thesis seeks to complement and extend these findings by investigating the roles of the perceptual and action brain systems in cue reactivity of nicotine addiction. First, Chapter 2 reviews cortical and cerebellar neuroimaging studies in the context of addiction with a particular focus on the sensory and motor aspects of the disease. Sensory, sensorimotor and motor brain regions are activated by drug-related stimuli in addicts, and their activations in response to drug cues can predict relapse and correlate with craving, severity of dependence and automatized behavioral reactions towards the stimuli. Furthermore, we propose a model of how sensory and motor processes might be involved in the different stages of addiction.

Chapter 3 deals with the sensorimotor aspects of the habitual mechanisms in nicotine addiction. We showed higher fMRI activations in smokers than nonsmokers in response to smoking-related but not control images. These group differences were located not only in brain regions associated with craving and habitual learning, but also in brain regions responsible for the encoding of action knowledge and tool use skills (premotor cortex, superior parietal lobule, and right lateral cerebellum). Furthermore, in a behavioral orientation affordance paradigm smokers, but not non-smokers, showed an automatized responsiveness to smoking paraphernalia. These observations were corroborated by the strong intercorrelations between fMRI activations in tool use-related brain regions, behavioral responsiveness to smoking-related cues, and severity of nicotine dependence. Apparently, smoking-related action representations in smokers are stored in brain regions typically representing tool use skills and action knowledge.

While cue reactivity is one of the main paradigms used in addiction research, many of the neuroimaging studies have yielded rather inconsistent results. Interestingly, the use of different sensory modalities for the presentation of drug cues has rarely been considered as a possible source of these inconsistencies. In Chapter 4 we addressed this issue in the context of smoking by testing whether neural responses to drug cues are modulated by the sensory modality of the presented stimuli. We measured brain activation with fMRI in smokers and nonsmokers while they either viewed images of smoking paraphernalia and control objects or touched the same objects without seeing them. Smoking-related stimuli presented in the haptic modality induced more pronounced neural cue reactivity than visual cues in the left dorsal striatum of smokers compared to non-smokers. Similarly, within-subject analyses in smokers revealed stronger neural cue reactivity for haptic stimuli in the left dorsal striatum as well as in the right premotor cortex and dorsolateral prefrontal cortex. A possible explanation for these results is that the perceived drug availability is higher for haptic than visual smoking cues and that 
haptic in comparison to visual perception is more strongly linked to habits and automatized action schemata. Furthermore, an increased fMRI cue reactivity for visual compared to haptic smoking cues was found in the right cerebellum of smokers. Our findings clearly demonstrate that cue reactivity of brain regions involved in crucial addiction-related processes such as habits and automatized action schemata as well as reward motivation and decision-making is modulated by the sensory modality in which the smoking cues are presented. This insight could be used for the development of more reliable fMRI-specific biomarkers, more ecologically valid experimental designs, and more effective cue-exposure therapies of addiction.

Chapter 5 provides evidence that not only unisensory but also multisensory processes are essential for understanding the neuroplasticity of addiction. In an fMRI study we demonstrated that in the lateral occipital complex, a cortical region crucial for crossmodal object recognition, the visuo-haptic integration pattern for smoking paraphernalia but not for control objects is strongly correlated with the individual severity of nicotine addiction. Heavier smokers showed more pronounced visuo-haptic integration for smoking paraphernalia in comparison to light smokers or non-smokers. These results indicate that prolonged addictionrelated perceptual experience facilitates the merging of visual and haptic inputs in the lateral occipital complex. Efficient multisensory integration of drug-related cues may provide these objects with particular salience, possibly resulting in enhanced detection rates and orientation responses. An important conclusion is that perceptual processes such as multisensory integration can be successfully implemented as potential biological markers of severity of addiction. Additionally, the study provides an example of how basic multisensory integration research might benefit from studying clinical populations which exhibit compulsive behaviors (e.g. drug seeking and drug taking) characterized by an intensive crossmodal experience and multisensory integration expertise.

The main findings from Chapters 2-5, the implications for clinical practice and research as well as issues which should be addressed by future research are discussed in Chapter 6.

Although perception and action have been neglected by addiction research, the present thesis demonstrates that they play significant roles for nicotine addiction. Due to the regular and intensive engagement of smokers in smoking-related sensorimotor and multisensory activities (e.g., seeing, reaching for and manipulating cigarettes, lighters, ashtrays, etc.), the corresponding perceptual and action representations seem to be particularly enhanced on the neural and behavioral level. A clinically relevant observation is that the strengthened sensorimotor and multisensory representations are linked to the expression of smoking-related behavior and to external variables such as test scores indicating the individual severity of nicotine addiction. Implementing these findings in the future research and clinical practice should improve the outcomes of drug cue reactivity studies as well as of addiction treatment strategies. 

Samenvatting 


\section{Samenvatting}

Verslaving is een van de ziektes met de grootste uitwerking op de samenleving, zoals is af te meten aan het aantal verslavingsgerelateerde doden en de groeiende kosten voor de gezondheidszorg. Het doorgronden van het aan deze ziekte onderliggende mechanisme is een lastige opgave gebleken, maar de neurowetenschap heeft desalniettemin belangrijke stappen gezet die tonen dat drugsmisbruik processen kaapt die ten gronde liggen aan leren, geheugen en cognitieve controle, om compulsief drugsgebruik en drugszoekend gedrag te ontwikkelen dat goed bestand is tegen verandering (Hoofdstuk 1 bevat een kort overzicht van de bevindingen binnen de neurowetenschap van verslaving).

Dit proefschrift poogt deze bevindingen aan te vullen en uit te breiden door de rol van hersensystemen voor waarneming en handeling in cue reactiviteit van nicotine verslaving te onderzoeken. Eerst bespreekt Hoofdstuk $\mathbf{2}$ corticale en cerebellaire neuroimaging studies in het kader van verslaving, met een bijzondere nadruk op de zintuiglijke en motorische aspecten van de ziekte. Zintuiglijke, sensorimotorische en motorische gebieden van de hersenen worden geactiveerd door drugsgerelateerde stimuli in verslaafden, en hun activiteit in reactie op drugs cues kan terugval voorspellen en kan correleren met verlangen, de ernst van de afhankelijkheid en met geautomatiseerd gedrag ten opzichte van de stimuli. Verder introduceren we een model van hoe sensorische en motorische processen betrokken kunnen zijn bij de verschillende stadia van verslaving.

Hoofdstuk 3 behandelt de sensomotorische aspecten van gewoonte mechanismen in nicotine verslaving. We vonden hogere fMRI activatie in rokers dan niet-rokers in reactie op roken-gerelateerde afbeeldingen maar niet op controle afbeeldingen. Deze groepsverschillen bevonden zich niet alleen in hersengebieden die in verband worden gebracht met craving en het aanleren van gewoontes, maar ook in hersengebieden verantwoordelijk voor de representatie van handelingskennis en werktuiggebruiksvaardigheden (premotorische cortex, lobulus parietalis superior en rechter laterale cerebellum). Bovendien, in een gedragsoriëntatie-affordantie paradigma, toonden rokers, maar niet-rokers niet, een automatische reactie op rookgerei. Deze waarnemingen werden bevestigd door sterke correlaties tussen fMRI activeringen in werktuiggebruiksgerelateerde hersengebieden, gedrags reactiviteit op rokengerelateerde cues, en de ernst van de nicotine afhankelijkheid. Blijkbaar worden rokengerelateerde handelingsrepresentaties in rokers opgeslagen in hersengebieden die doorgaans werktuiggebruiksvaardigheden en handelingskennis vertegenwoordigen.

Terwijl cue reactiviteit een van de belangrijkste paradigma's is die gebruikt worden in verslavingsonderzoek, leverden veel van de neuroimaging studies inconsistente resultaten op. Interessant is dat het gebruik van verschillende zintuiglijke modaliteiten voor de presentatie van drugs cues zelden beschouwd is als mogelijke oorzaak van deze inconsistenties. In Hoofdstuk 4 hebben we deze kwestie behandeld in de context van roken door te testen of de neurale reacties op drugs cues worden gemoduleerd door de zintuiglijke modaliteit van de gepresenteerde stimuli. We meetten hersenactiviteit met fMRI bij rokers en nietrokers, terwijl ze ofwel beelden van rookgerei en controle objecten bekeken, of dezelfde objecten aanraakten zonder ze te zien. Roken gerelateerde stimuli die in de 
haptische modaliteit gepresenteerd werden leidden tot meer neurale cue reactiviteit dan visuele cues in het linker dorsale striatum van rokers in vergelijking met nietrokers. Ook within-subjects analyses in rokers onthulden sterkere neurale cue reactiviteit voor haptische stimuli in het linker dorsale striatum alsook in de rechter premotorische cortex en dorsolaterale prefrontale cortex. Een mogelijke verklaring voor deze resultaten is dat de waargenomen beschikbaarheid van de drug groter is voor haptische dan voor visuele rook cues, en dat haptische waarneming in vergelijking met visuele waarneming sterker verbonden is met gewoontes en geautomatiseerde actie schema's. Verder werd een verhoogde fMRI cue reactiviteit gevonden voor visuele rook cues vergeleken met haptische rook cues in het rechter cerebellum van rokers. Onze bevindingen laten duidelijk zien dat cue reactiviteit in hersengebieden betrokken bij cruciale verslavingsgerelateerde processen zoals gewoontes en geautomatiseerde actie schema's zowel als beloningsgestuurde motivatie en besluitvorming wordt gemoduleerd door de zintuiglijke modaliteit waarin de rook cues worden gepresenteerd. Dit inzicht zou kunnen worden gebruikt voor de ontwikkeling van betrouwbaardere fMRI-specifieke biologische markers, ecologisch meer valide experimentele opzetten, en effectievere cue blootstellings therapieën voor verslaving.

Hoofdstuk 5 levert bewijs dat niet alleen unisensorische maar ook multisensorische processen essentieel zijn voor het begrip van de neuroplasticiteit van verslaving. In een fMRI studie toonden we dat in het laterale occipitale complex, een corticaal gebied cruciaal voor crossmodale objectherkenning, het visueel-haptische integratie effect voor rookgerei maar niet voor controle objecten sterk correleert met de individuele graad van nicotine verslaving. Zwaardere rokers toonden meer uitgesproken visueel-haptische integratie voor rookgerei dan lichte of niet-rokers. Deze resultaten duiden op een vergemakkelijking van het samenvoegen van haptische en visuele invoer in het laterale occipitale complex door langdurige ervaring van verslavingsgerelateerde waarneming. Efficiënte multisensorische integratie van drugsgerelateerde cues zou deze objecten kunnen voorzien van bijzondere saillantie, mogelijk resulterend in verbeterde detectie kansen en oriëntatie reacties. Een belangrijke conclusie is dat waarnemingsprocessen, zoals multisensorische integratie succesvol kunnen worden geïmplementeerd als potentiële biologische markers van de ernst van de verslaving. Verder geeft de studie een voorbeeld van hoe fundamenteel multisensorische integratie onderzoek zou kunnen profiteren van het bestuderen van klinische populaties die dwangmatig gedrag vertonen (zoals drugs zoekend gedrag en drugsgebruik) gekenmerkt door een intensieve crossmodale ervaring en multisensorische integratie-expertise.

De belangrijkste bevindingen uit de Hoofdstukken 2-5, de implicaties voor de klinische praktijk en onderzoek alsmede kwesties die moeten worden aangepakt door toekomstig onderzoek worden besproken in Hoofdstuk 6.

Hoewel perceptie en actie zijn verwaarloosd door verslavingsonderzoek, toont dit proefschrift aan dat ze een belangrijke rol spelen in nicotine verslaving. Door de herhaaldelijke en intensieve betrokkenheid van rokers met rokengerelateerde sensorimotorische en multisensorische activiteiten (bv., zien, reiken naar en het manipuleren van sigaretten, aanstekers, asbakken, enz.), lijken de bijbehorende waarnemings en actievoorstellingen met name op het neurale en gedragsmatige niveau te worden versterkt. Een klinisch relevante observatie is dat de versterkte sensorimotorische en multisensorische voorstellingen zijn gekoppeld 
aan de uitdrukking van roken gerelateerd gedrag en aan externe variabelen zoals testscores die de individuele ernst van de nicotineverslaving weergeven. De verwezenlijking van deze bevindingen in toekomstig onderzoek en de klinische praktijk zou de uitkomsten van zowel drugs cue reactiviteit studies als van verslavingstherapie strategieën moeten verbeteren. 
References 


\section{References}

Amedi A, Malach R, Hendler T, Peled S, Zohary E (2001) Visuo-haptic objectrelated activation in the ventral visual pathway. Nat Neurosci 4:324-330.

Amedi A, Jacobson G, Hendler T, Malach R, Zohary E (2002) Convergence of visual and tactile shape processing in the human lateral occipital complex. Cereb Cortex 12:1202-1212.

Amedi A, von Kriegstein K, van Atteveldt NM, Beauchamp MS, Naumer MJ (2005) Functional imaging of human crossmodal identification and object recognition. Exp Brain Res 166:559-571.

Amedi A, Stern WM, Camprodon JA, Bermpohl F, Merabet L, Rotman S, Hemond C, Meijer P, Pascual-Leone A (2007) Shape conveyed by visual-to-auditory sensory substitution activates the lateral occipital complex. Nat Neurosci 10:687-689.

APA (2000) Diagnostic and Statistical Manual of Mental Disorders DSM-IV-TR Fourth Edition (Text Revision): American Psychiatric Association.

Arbib MA, Billard A, Iacoboni M, Oztop E (2000) Synthetic brain imaging: grasping, mirror neurons and imitation. Neural Netw 13:975-997.

Bailey SR, Goedeker KC, Tiffany ST (2010) The impact of cigarette deprivation and cigarette availability on cue-reactivity in smokers. Addiction (Abingdon, England) 105:364-372.

Baler RD, Volkow ND (2006) Drug addiction: the neurobiology of disrupted selfcontrol. Trends Mol Med 12:559-566.

Batterson VG, Rose SA, Yonas A, Grant KS, Sackett GP (2008) The effect of experience on the development of tactual-visual transfer in pigtailed macaque monkeys. Dev Psychobiol 50:88-96.

Bavelier D, Neville HJ (2002) Cross-modal plasticity: where and how? Nat Rev Neurosci 3:443-452.

Bavelier D, Dye MW, Hauser PC (2006) Do deaf individuals see better? Trends Cogn Sci 10:512-518.

Baxter BW, Hinson RE (2001) Is smoking automatic? Demands of smoking behavior on attentional resources. J Abnorm Psychol 110:59-66.

Beauchamp MS (2005) Statistical criteria in FMRI studies of multisensory integration. Neuroinformatics 3:93-113.

Bechara A, Damasio H, Damasio AR, Lee GP (1999) Different contributions of the human amygdala and ventromedial prefrontal cortex to decision-making. J Neurosci 19:5473-5481.

Belin D, Everitt BJ (2008) Cocaine seeking habits depend upon dopaminedependent serial connectivity linking the ventral with the dorsal striatum. Neuron 57:432-441.

Belin D, Jonkman S, Dickinson A, Robbins TW, Everitt BJ (2009) Parallel and interactive learning processes within the basal ganglia: relevance for the understanding of addiction. Behav Brain Res 199:89-102.

Berridge KC (2000) Measuring hedonic impact in animals and infants: microstructure of affective taste reactivity patterns. Neurosci Biobehav Rev 24:173-198. 
Berridge KC (2007) The debate over dopamine's role in reward: the case for incentive salience. Psychopharmacology (Berl) 191:391-431.

Bleich S, Havemann-Reinecke U, Kornhuber J (2002) Fagerström-Test für Nikotinabhängigkeit (FTNA): Göttingen: Beltz Test GmbH.

Boileau I, Dagher A, Leyton M, Gunn RN, Baker GB, Diksic M, Benkelfat C (2006) Modeling sensitization to stimulants in humans: an [11C]raclopride/positron emission tomography study in healthy men. Arch Gen Psychiatry 63:1386-1395.

Breiter HC, Gollub RL, Weisskoff RM, Kennedy DN, Makris N, Berke JD, Goodman JM, Kantor HL, Gastfriend DR, Riorden JP, Mathew RT, Rosen BR, Hyman SE (1997) Acute effects of cocaine on human brain activity and emotion. Neuron 19:591-611.

Brody AL (2006) Functional brain imaging of tobacco use and dependence. J Psychiatr Res 40:404-418.

Brody AL, Mandelkern MA, London ED, Childress AR, Lee GS, Bota RG, Ho ML, Saxena S, Baxter LR, Jr., Madsen D, Jarvik ME (2002) Brain metabolic changes during cigarette craving. Arch Gen Psychiatry 59:1162-1172.

Brody AL, Mandelkern MA, Olmstead RE, Jou J, Tiongson E, Allen V, Scheibal D, London ED, Monterosso JR, Tiffany ST, Korb A, Gan JJ, Cohen MS (2007) Neural substrates of resisting craving during cigarette cue exposure. Biol Psychiatry 62:642-651.

Brosch M, Selezneva E, Scheich H (2005) Nonauditory events of a behavioral procedure activate auditory cortex of highly trained monkeys. J Neurosci 25:6797-6806.

Buneo CA, Andersen RA (2006) The posterior parietal cortex: sensorimotor interface for the planning and online control of visually guided movements. Neuropsychologia 44:2594-2606.

Bushnell EW, Baxt C (1999) Children's haptic and cross-modal recognition with familiar and unfamiliar objects. Journal of experimental psychology Human perception and performance 25:1867-1881.

Cagniard B, Balsam PD, Brunner D, Zhuang X (2006) Mice with chronically elevated dopamine exhibit enhanced motivation, but not learning, for a food reward. Neuropsychopharmacology 31:1362-1370.

Calvo-Merino B, Glaser DE, Grezes J, Passingham RE, Haggard P (2005) Action observation and acquired motor skills: an FMRI study with expert dancers. Cereb Cortex 15:1243-1249.

Calvo-Merino B, Grezes J, Glaser DE, Passingham RE, Haggard P (2006) Seeing or doing? Influence of visual and motor familiarity in action observation. Curr Biol 16:1905-1910.

Carter BL, Tiffany ST (1999) Meta-analysis of cue-reactivity in addiction research. Addiction 94:327-340.

Carter BL, Tiffany ST (2001) The cue-availability paradigm: the effects of cigarette availability on cue reactivity in smokers. Exp Clin Psychopharmacol 9:183190.

Chaminade T, Meltzoff AN, Decety J (2005) An fMRI study of imitation: action representation and body schema. Neuropsychologia 43:115-127.

Chao LL, Martin A (2000) Representation of manipulable man-made objects in the dorsal stream. Neuroimage 12:478-484. 
Chaudhri N, Sahuque LL, Janak PH (2008) Context-induced relapse of conditioned behavioral responding to ethanol cues in rats. Biol Psychiatry 64:203-210.

Chiamulera C (2005) Cue reactivity in nicotine and tobacco dependence: a "multiple-action" model of nicotine as a primary reinforcement and as an enhancer of the effects of smoking-associated stimuli. Brain Res Brain Res Rev 48:74-97.

Conklin CA, Tiffany ST (2002) Applying extinction research and theory to cueexposure addiction treatments. Addiction 97:155-167.

Cools R, Clark L, Owen AM, Robbins TW (2002) Defining the neural mechanisms of probabilistic reversal learning using event-related functional magnetic resonance imaging. J Neurosci 22:4563-4567.

Cox SM, Benkelfat C, Dagher A, Delaney JS, Durand F, McKenzie SA, Kolivakis T, Casey KF, Leyton M (2009) Striatal dopamine responses to intranasal cocaine self-administration in humans. Biol Psychiatry 65:846-850.

Creem-Regehr SH, Lee JN (2005) Neural representations of graspable objects: are tools special? Brain Res Cogn Brain Res 22:457-469.

Crombag HS, Gorny G, Li Y, Kolb B, Robinson TE (2005) Opposite effects of amphetamine self-administration experience on dendritic spines in the medial and orbital prefrontal cortex. Cereb Cortex 15:341-348.

D'Ausilio A, Altenmuller E, Olivetti Belardinelli M, Lotze M (2006) Cross-modal plasticity of the motor cortex while listening to a rehearsed musical piece. Eur J Neurosci 24:955-958.

Dani JA, Ji D, Zhou FM (2001) Synaptic plasticity and nicotine addiction. Neuron 31:349-352.

Devonshire IM, Mayhew JE, Overton PG (2007) Cocaine preferentially enhances sensory processing in the upper layers of the primary sensory cortex. Neuroscience 146:841-851.

Devonshire IM, Berwick J, Jones M, Martindale J, Johnston D, Overton PG, Mayhew JE (2004) Haemodynamic responses to sensory stimulation are enhanced following acute cocaine administration. Neuroimage 22:17441753.

Dom G, Sabbe B, Hulstijn W, van den Brink W (2005) Substance use disorders and the orbitofrontal cortex: systematic review of behavioural decision-making and neuroimaging studies. Br J Psychiatry 187:209-220.

Drevets WC, Price JC, Kupfer DJ, Kinahan PE, Lopresti B, Holt D, Mathis C (1999) PET measures of amphetamine-induced dopamine release in ventral versus dorsal striatum. Neuropsychopharmacology 21:694-709.

Drevets WC, Gautier C, Price JC, Kupfer DJ, Kinahan PE, Grace AA, Price JL, Mathis CA (2001) Amphetamine-induced dopamine release in human ventral striatum correlates with euphoria. Biol Psychiatry 49:81-96.

Droungas A, Ehrman RN, Childress AR, O'Brien CP (1995) Effect of smoking cues and cigarette availability on craving and smoking behavior. Addict Behav 20:657-673.

Ellis R, Tucker M (2000) Micro-affordance: the potentiation of components of action by seen objects. Br J Psychol 91 ( Pt 4):451-471.

Ernst MO (2007) Learning to integrate arbitrary signals from vision and touch. J Vis 7:7 1-14. 
Everitt BJ, Robbins TW (2005) Neural systems of reinforcement for drug addiction: from actions to habits to compulsion. Nat Neurosci 8:1481-1489.

Fecteau S, Fregni F, Boggio PS, Camprodon JA, Pascual-Leone A (2010) Neuromodulation of decision-making in the addictive brain. Subst Use Misuse 45:1766-1786.

Fecteau S, Pascual-Leone A, Zald DH, Liguori P, Theoret H, Boggio PS, Fregni F (2007) Activation of prefrontal cortex by transcranial direct current stimulation reduces appetite for risk during ambiguous decision making. J Neurosci 27:6212-6218.

Felleman DJ, Van Essen DC (1991) Distributed hierarchical processing in the primate cerebral cortex. Cereb Cortex 1:1-47.

Field M (2006a) Attentional biases in drug abuse and addiction: cognitive mechanisms, causes, consequences, and implications. In: Cognition and Addiction (Munafo M, Albery IP, eds), pp 73-99. Oxford: Oxford University Press.

Field M, Cox WM (2008) Attentional bias in addictive behaviors: a review of its development, causes, and consequences. Drug Alcohol Depend 97:1-20.

Field M, Mogg K, Bradley BP (2006b) Automaticity of smoking behaviour: the relationship between dual-task performance, daily cigarette intake and subjective nicotine effects. J Psychopharmacol 20:799-805.

Fink GR, Marshall JC, Halligan PW, Frith CD, Driver J, Frackowiak RS, Dolan RJ (1999) The neural consequences of conflict between intention and the senses. Brain 122 ( Pt 3):497-512.

Finney EM, Fine I, Dobkins KR (2001) Visual stimuli activate auditory cortex in the deaf. Nat Neurosci 4:1171-1173.

Fogassi L, Ferrari PF, Gesierich B, Rozzi S, Chersi F, Rizzolatti G (2005) Parietal lobe: from action organization to intention understanding. Science 308:662667.

Forman SD, Cohen JD, Fitzgerald M, Eddy WF, Mintun MA, Noll DC (1995) Improved assessment of significant activation in functional magnetic resonance imaging (fMRI): use of a cluster-size threshold. Magn Reson Med 33:636-647.

Fowler JS, Volkow ND, Kassed CA, Chang L (2007) Imaging the addicted human brain. Sci Pract Perspect 3:4-16.

Franken IH (2003) Drug craving and addiction: integrating psychological and neuropsychopharmacological approaches. Prog Neuropsychopharmacol Biol Psychiatry 27:563-579.

Fregni F, Liguori P, Fecteau S, Nitsche MA, Pascual-Leone A, Boggio PS (2008) Cortical stimulation of the prefrontal cortex with transcranial direct current stimulation reduces cue-provoked smoking craving: a randomized, shamcontrolled study. J Clin Psychiatry 69:32-40.

Garavan H, Hester R (2007) The role of cognitive control in cocaine dependence. Neuropsychol Rev 17:337-345.

Garavan H, Pankiewicz J, Bloom A, Cho JK, Sperry L, Ross TJ, Salmeron BJ, Risinger R, Kelley D, Stein EA (2000) Cue-induced cocaine craving: neuroanatomical specificity for drug users and drug stimuli. Am J Psychiatry 157:1789-1798. 
Gerdeman GL, Partridge JG, Lupica CR, Lovinger DM (2003) It could be habit forming: drugs of abuse and striatal synaptic plasticity. Trends Neurosci 26:184-192.

Gilbert DG, Rabinovich NE (2006) International Smoking Image Series (With Neutral Counterparts). Carbondale, Integrative Neuroscience Laboratory, Department of Psychology, Southern Illinois University.

Gloria R, Angelos L, Schaefer HS, Davis JM, Majeskie M, Richmond BS, Curtin JJ, Davidson RJ, Baker TB (2009) An fMRI investigation of the impact of withdrawal on regional brain activity during nicotine anticipation. Psychophysiology 46:681-693.

Goebel R, Esposito F, Formisano E (2006) Analysis of functional image analysis contest (FIAC) data with brainvoyager QX: From single-subject to cortically aligned group general linear model analysis and self-organizing group independent component analysis. Hum Brain Mapp 27:392-401.

Goldreich D, Kanics IM (2003) Tactile acuity is enhanced in blindness. J Neurosci 23:3439-3445.

Goldstein RZ, Craig AD, Bechara A, Garavan H, Childress AR, Paulus MP, Volkow ND (2009) The neurocircuitry of impaired insight in drug addiction. Trends Cogn Sci 13:372-380.

Gonzalo D, Shallice T, Dolan R (2000) Time-dependent changes in learning audiovisual associations: a single-trial fMRI study. Neuroimage 11:243255.

Grabowski TJ, Damasio H, Damasio AR (1998) Premotor and prefrontal correlates of category-related lexical retrieval. Neuroimage 7:232-243.

Grafton ST, Fadiga L, Arbib MA, Rizzolatti G (1997) Premotor cortex activation during observation and naming of familiar tools. Neuroimage 6:231-236.

Grant S, London ED, Newlin DB, Villemagne VL, Liu X, Contoreggi C, Phillips RL, Kimes AS, Margolin A (1996) Activation of memory circuits during cue-elicited cocaine craving. Proc Natl Acad Sci U S A 93:12040-12045.

Graziano MS, Aflalo TN (2007) Mapping behavioral repertoire onto the cortex. Neuron 56:239-251.

Grezes J, Decety J (2002) Does visual perception of object afford action? Evidence from a neuroimaging study. Neuropsychologia 40:212-222.

Grezes J, Tucker M, Armony J, Ellis R, Passingham RE (2003) Objects automatically potentiate action: an fMRI study of implicit processing. Eur J Neurosci 17:2735-2740.

Grol MJ, de Lange FP, Verstraten FA, Passingham RE, Toni I (2006) Cerebral changes during performance of overlearned arbitrary visuomotor associations. J Neurosci 26:117-125.

Grusser SM, Wrase J, Klein S, Hermann D, Smolka MN, Ruf M, Weber-Fahr W, Flor H, Mann K, Braus DF, Heinz A (2004) Cue-induced activation of the striatum and medial prefrontal cortex is associated with subsequent relapse in abstinent alcoholics. Psychopharmacology (Berl) 175:296-302.

Haaland KY, Harrington DL, Knight RT (2000) Neural representations of skilled movement. Brain 123 ( Pt 11):2306-2313.

Hackley SA (2009) The speeding of voluntary reaction by a warning signal. Psychophysiology 46:225-233. 
Haxby JV, Gobbini MI, Furey ML, Ishai A, Schouten JL, Pietrini P (2001) Distributed and overlapping representations of faces and objects in ventral temporal cortex. Science 293:2425-2430.

Heinze M, Wolfling K, Grusser SM (2007) Cue-induced auditory evoked potentials in alcoholism. Clin Neurophysiol 118:856-862.

Hester R, Garavan H (2004) Executive dysfunction in cocaine addiction: evidence for discordant frontal, cingulate, and cerebellar activity. J Neurosci 24:11017-11022.

Higuchi S, Imamizu H, Kawato M (2007) Cerebellar activity evoked by common tool-use execution and imagery tasks: an fMRI study. Cortex 43:350-358.

Hogarth LC, Mogg K, Bradley BP, Duka T, Dickinson A (2003) Attentional orienting towards smoking-related stimuli. Behav Pharmacol 14:153-160.

Hötting K, Röder B (2009) Auditory and auditory-tactile processing in congenitally blind humans. Hear Res 258:165-174.

http://www.who.int/substance_abuse/facts/global_burden/en/index.html (online access on 29.03.2010) The global burden. In: World Health Organization's Homepage.

Hui GK, Wong KL, Chavez CM, Leon MI, Robin KM, Weinberger NM (2009) Conditioned tone control of brain reward behavior produces highly specific representational gain in the primary auditory cortex. Neurobiol Learn Mem 92:27-34.

Hyde KL, Lerch J, Norton A, Forgeard M, Winner E, Evans AC, Schlaug G (2009) The effects of musical training on structural brain development: a longitudinal study. Ann N Y Acad Sci 1169:182-186.

Hyman SE (2005) Addiction: a disease of learning and memory. Am J Psychiatry 162:1414-1422.

Hyman SE (2007) The neurobiology of addiction: implications for voluntary control of behavior. Am J Bioeth 7:8-11.

Hyman SE, Malenka RC (2001) Addiction and the brain: the neurobiology of compulsion and its persistence. Nat Rev Neurosci 2:695-703.

Hyman SE, Malenka RC, Nestler EJ (2006) Neural mechanisms of addiction: the role of reward-related learning and memory. Annu Rev Neurosci 29:565598.

Imamizu H, Kuroda T, Miyauchi S, Yoshioka T, Kawato M (2003) Modular organization of internal models of tools in the human cerebellum. Proc Natl Acad Sci U S A 100:5461-5466.

Imamizu H, Miyauchi S, Tamada T, Sasaki Y, Takino R, Putz B, Yoshioka T, Kawato M (2000) Human cerebellar activity reflecting an acquired internal model of a new tool. Nature 403:192-195.

James TW, Humphrey GK, Gati JS, Servos P, Menon RS, Goodale MA (2002) Haptic study of three-dimensional objects activates extrastriate visual areas. Neuropsychologia 40:1706-1714.

Jeannerod M, Jacob P (2005) Visual cognition: a new look at the two-visual systems model. Neuropsychologia 43:301-312.

Jiang X, Bradley E, Rini RA, Zeffiro T, Vanmeter J, Riesenhuber M (2007) Categorization training results in shape- and category-selective human neural plasticity. Neuron 53:891-903. 
Johnson-Frey SH (2004) The neural bases of complex tool use in humans. Trends Cogn Sci 8:71-78.

Johnson-Frey SH, Newman-Norlund R, Grafton ST (2005) A distributed left hemisphere network active during planning of everyday tool use skills. Cereb Cortex 15:681-695.

Johnson BA, Chen YR, Schmitz J, Bordnick P, Shafer A (1998) Cue reactivity in cocaine-dependent subjects: effects of cue type and cue modality. Addict Behav 23:7-15.

Jones S, Bonci A (2005) Synaptic plasticity and drug addiction. Curr Opin Pharmacol 5:20-25.

Kalivas PW (2009) The glutamate homeostasis hypothesis of addiction. Nat Rev Neurosci 10:561-572.

Kalivas PW, Volkow ND (2005) The neural basis of addiction: a pathology of motivation and choice. Am J Psychiatry 162:1403-1413.

Kalivas PW, Volkow N, Seamans J (2005) Unmanageable motivation in addiction: a pathology in prefrontal-accumbens glutamate transmission. Neuron 45:647650.

Kapogiannis D, Campion P, Grafman J, Wassermann EM (2008) Reward-related activity in the human motor cortex. Eur J Neurosci 27:1836-1842.

Kastner S, Ungerleider LG (2000) Mechanisms of visual attention in the human cortex. Annu Rev Neurosci 23:315-341.

Kauer JA, Malenka RC (2007) Synaptic plasticity and addiction. Nat Rev Neurosci 8:844-858.

Kelley AE, Berridge KC (2002) The neuroscience of natural rewards: relevance to addictive drugs. J Neurosci 22:3306-3311.

Kim S, James TW (2009) Enhanced effectiveness in visuo-haptic object-selective brain regions with increasing stimulus salience. Hum Brain Mapp.

Koob GF, Volkow ND (2010) Neurocircuitry of Addiction (vol 35, pg 217, 2010). Neuropsychopharmacology 35:1051-1051.

Kosten TR, Scanley BE, Tucker KA, Oliveto A, Prince C, Sinha R, Potenza MN, Skudlarski P, Wexler BE (2006) Cue-induced brain activity changes and relapse in cocaine-dependent patients. Neuropsychopharmacology 31:644650.

Kourtzi Z, DiCarlo JJ (2006) Learning and neural plasticity in visual object recognition. Curr Opin Neurobiol 16:152-158.

Kubler A, Dixon V, Garavan H (2006) Automaticity and reestablishment of executive control-an fMRI study. J Cogn Neurosci 18:1331-1342.

Lacey S, Campbell C (2006) Mental representation in visual/haptic crossmodal memory: evidence from interference effects. Quarterly journal of experimental psychology (2006) 59:361-376.

Lacey S, Tal N, Amedi A, Sathian K (2009) A putative model of multisensory object representation. Brain Topogr 21:269-274.

Lacourse MG, Orr EL, Cramer SC, Cohen MJ (2005) Brain activation during execution and motor imagery of novel and skilled sequential hand movements. Neuroimage 27:505-519.

Lamb RJ, Preston KL, Schindler CW, Meisch RA, Davis F, Katz JL, Henningfield JE, Goldberg SR (1991) The reinforcing and subjective effects of morphine 
in post-addicts: a dose-response study. J Pharmacol Exp Ther 259:11651173.

Lang CE, Bastian AJ (2002) Cerebellar damage impairs automaticity of a recently practiced movement. J Neurophysiol 87:1336-1347.

Lee D, Seo H (2007) Mechanisms of reinforcement learning and decision making in the primate dorsolateral prefrontal cortex. Ann N Y Acad Sci 1104:108122.

Lewis JW (2006) Cortical networks related to human use of tools. Neuroscientist 12:211-231.

Leyton M, Boileau I, Benkelfat C, Diksic M, Baker G, Dagher A (2002) Amphetamine-induced increases in extracellular dopamine, drug wanting, and novelty seeking: a PET/[11C]raclopride study in healthy men. Neuropsychopharmacology 27:1027-1035.

Logan GD (1988) Toward an instance theory of automatization. Psychol Rev 95:577-621.

Makin TR, Holmes NP, Ehrsson HH (2008) On the other hand: dummy hands and peripersonal space. Behav Brain Res 191:1-10.

Malach R, Reppas JB, Benson RR, Kwong KK, Jiang H, Kennedy WA, Ledden PJ, Brady TJ, Rosen BR, Tootell RB (1995) Object-related activity revealed by functional magnetic resonance imaging in human occipital cortex. Proc Natl Acad Sci U S A 92:8135-8139.

Malouin F, Richards CL, Jackson PL, Dumas F, Doyon J (2003) Brain activations during motor imagery of locomotor-related tasks: a PET study. Hum Brain Mapp 19:47-62.

Mansour A, Watson SJ (2000) Dopamine Receptor Expression in the Central Nervous System In: Psychopharmacology: the Fourth Generation of Progress (Bloom FE, Kupfer DJ, eds): Lippincott Williams \& Wilkins; 4th edition.

McAuliffe WE, Gordon RA (1974) A test of Lindesmith's theory of addiction: the frequency of euphoria among long-term addicts. AJS 79:795-840.

McBride D, Barrett SP, Kelly JT, Aw A, Dagher A (2006) Effects of expectancy and abstinence on the neural response to smoking cues in cigarette smokers: an fMRI study. Neuropsychopharmacology 31:2728-2738.

McClernon FJ, Kozink RV, Lutz AM, Rose JE (2009) 24-h smoking abstinence potentiates fMRI-BOLD activation to smoking cues in cerebral cortex and dorsal striatum. Psychopharmacology (Berl) 204:25-35.

Merabet LB, Pascual-Leone A (2010) Neural reorganization following sensory loss: the opportunity of change. Nat Rev Neurosci 11:44-52.

Meredith MA, Stein BE (1986) Visual, auditory, and somatosensory convergence on cells in superior colliculus results in multisensory integration. J Neurophysiol 56:640-662.

Metherate R (2004) Nicotinic acetylcholine receptors in sensory cortex. Learn Mem 11:50-59.

Miller NS, Gold MS (1994) Dissociation of "conscious desire" (craving) from and relapse in alcohol and cocaine dependence. Ann Clin Psychiatry 6:99-106.

Mogg K, Field M, Bradley BP (2005) Attentional and approach biases for smoking cues in smokers: an investigation of competing theoretical views of addiction. Psychopharmacology (Berl) 180:333-341. 
Monfils MH, Plautz EJ, Kleim JA (2005) In search of the motor engram: motor map plasticity as a mechanism for encoding motor experience. Neuroscientist 11:471-483.

Montague PR, Hyman SE, Cohen JD (2004) Computational roles for dopamine in behavioural control. Nature 431:760-767.

Morris JS, Dolan RJ (2004) Dissociable amygdala and orbitofrontal responses during reversal fear conditioning. Neuroimage 22:372-380.

Muller CP, Huston JP (2007) Dopamine activity in the occipital and temporal cortices of rats: dissociating effects of sensory but not pharmacological stimulation. Synapse 61:254-258.

Musacchia G, Sams M, Skoe E, Kraus N (2007) Musicians have enhanced subcortical auditory and audiovisual processing of speech and music. Proc Natl Acad Sci U S A 104:15894-15898.

Nakashita S, Saito DN, Kochiyama T, Honda M, Tanabe HC, Sadato N (2008) Tactile-visual integration in the posterior parietal cortex: a functional magnetic resonance imaging study. Brain Res Bull 75:513-525.

Naqvi NH, Bechara A (2009) The hidden island of addiction: the insula. Trends Neurosci 32:56-67.

Naqvi NH, Rudrauf D, Damasio H, Bechara A (2007) Damage to the insula disrupts addiction to cigarette smoking. Science 315:531-534.

Naumer MJ, Kaiser J (2010) Multisensory object perception in the primate brain. New York: Springer.

Naumer MJ, van den Bosch JJF, Polony A, Kaiser J (2010b) Multisensory functional magnetic resonance imaging. In: Multisensory object perception in the primate brain. (Naumer M, Kaiser J, eds). New York: Springer.

Naumer MJ, Doehrmann O, Muller NG, Muckli L, Kaiser J, Hein G (2009) Cortical plasticity of audio-visual object representations. Cereb Cortex 19:16411653.

Naumer MJ, Ratz L, Yalachkov Y, Polony A, Doehrmann O, Ven Vvd, Müller NG, Kaiser J, Hein G (2010a) Visuohaptic convergence in a corticocerebellar network. European Journal of Neuroscience 31:1730-1736.

Ogiso T, Kobayashi K, Sugishita M (2000) The precuneus in motor imagery: a magnetoencephalographic study. Neuroreport 11:1345-1349.

Op de Beeck HP, Baker CI, DiCarlo JJ, Kanwisher NG (2006) Discrimination training alters object representations in human extrastriate cortex. J Neurosci 26:13025-13036.

Packard MG, Knowlton BJ (2002) Learning and memory functions of the Basal Ganglia. Annu Rev Neurosci 25:563-593.

Pantev C, Ross B, Fujioka T, Trainor LJ, Schulte M, Schulz M (2003) Music and learning-induced cortical plasticity. Ann N Y Acad Sci 999:438-450.

Pantoja J, Ribeiro S, Wiest M, Soares E, Gervasoni D, Lemos NA, Nicolelis MA (2007) Neuronal activity in the primary somatosensory thalamocortical loop is modulated by reward contingency during tactile discrimination. $\mathrm{J}$ Neurosci 27:10608-10620.

Park MS, Sohn JH, Suk JA, Kim SH, Sohn S, Sparacio R (2007) Brain substrates of craving to alcohol cues in subjects with alcohol use disorder. Alcohol Alcohol 42:417-422. 
Park SQ, Kahnt T, Beck A, Cohen MX, Dolan RJ, Wrase J, Heinz A (2010) Prefrontal cortex fails to learn from reward prediction errors in alcohol dependence. J Neurosci 30:7749-7753.

Pearce AJ, Thickbroom GW, Byrnes ML, Mastaglia FL (2000) Functional reorganisation of the corticomotor projection to the hand in skilled racquet players. Exp Brain Res 130:238-243.

Piasecki TM (2006) Relapse to smoking. Clin Psychol Rev 26:196-215.

Piasecki TM, Richardson AE, Smith SM (2007) Self-monitored motives for smoking among college students. Psychol Addict Behav 21:328-337.

Pietrini P, Furey ML, Ricciardi E, Gobbini MI, Wu WH, Cohen L, Guazzelli M, Haxby JV (2004) Beyond sensory images: Object-based representation in the human ventral pathway. Proc Natl Acad Sci U S A 101:5658-5663.

Pleger B, Blankenburg F, Ruff CC, Driver J, Dolan RJ (2008) Reward facilitates tactile judgments and modulates hemodynamic responses in human primary somatosensory cortex. J Neurosci 28:8161-8168.

Poldrack RA, Sabb FW, Foerde K, Tom SM, Asarnow RF, Bookheimer SY, Knowlton BJ (2005) The neural correlates of motor skill automaticity. J Neurosci 25:5356-5364.

Redish AD (2004) Addiction as a computational process gone awry. Science 306:1944-1947.

Reid MS, Flammino F, Starosta A, Palamar J, Franck J (2006) Physiological and subjective responding to alcohol cue exposure in alcoholics and control subjects: evidence for appetitive responding. J Neural Transm 113:15191535.

Riggio L, Iani C, Gherri E, Benatti F, Rubichi S, Nicoletti R (2008) The role of attention in the occurrence of the affordance effect. Acta Psychol (Amst) 127:449-458.

Robbins TW, Ersche KD, Everitt BJ (2008) Drug addiction and the memory systems of the brain. Ann N Y Acad Sci 1141:1-21.

Robinson TE, Berridge KC (1993) The neural basis of drug craving: an incentivesensitization theory of addiction. Brain Res Brain Res Rev 18:247-291.

Robinson TE, Berridge KC (2001) Incentive-sensitization and addiction. Addiction 96:103-114.

Russ MO, Mack W, Grama CR, Lanfermann H, Knopf M (2003) Enactment effect in memory: evidence concerning the function of the supramarginal gyrus. Exp Brain Res 149:497-504.

Russo SJ, Dietz DM, Dumitriu D, Morrison JH, Malenka RC, Nestler EJ (2010) The addicted synapse: mechanisms of synaptic and structural plasticity in nucleus accumbens. Trends Neurosci.

Saito DN, Okada T, Honda M, Yonekura Y, Sadato N (2006) Practice makes perfect: the neural substrates of tactile discrimination by Mah-Jong experts include the primary visual cortex. BMC Neurosci 7:79.

Sanes JN, Donoghue JP (2000) Plasticity and primary motor cortex. Annu Rev Neurosci 23:393-415.

Schmidbauer W, VomScheidt J (2004) Handbuch der Rauschdrogen. Frankfurt: Fischer (Tb.).

Schneider F, Habel U, Wagner M, Franke P, Salloum JB, Shah NJ, Toni I, Sulzbach C, Honig K, Maier W, Gaebel W, Zilles K (2001) Subcortical correlates of 
craving in recently abstinent alcoholic patients. Am J Psychiatry 158:10751083.

Schneider W, Shiffrin RM (1977) Controlled and automatic human information processing: I. Detection, search, and attention. Psychol Rev 84:1-66.

Schoenbaum G, Shaham Y (2007) The Role of Orbitofrontal Cortex in Drug Addiction: A Review of Preclinical Studies. Biol Psychiatry.

Schoenbaum G, Roesch MR, Stalnaker TA (2006) Orbitofrontal cortex, decisionmaking and drug addiction. Trends Neurosci 29:116-124.

Schultz W (2007a) Multiple dopamine functions at different time courses. Annu Rev Neurosci 30:259-288.

Schultz W (2007b) Behavioral dopamine signals. Trends Neurosci 30:203-210.

Schultz W, Dickinson A (2000) Neuronal coding of prediction errors. Annu Rev Neurosci 23:473-500.

Schultz W, Dayan P, Montague PR (1997) A neural substrate of prediction and reward. Science 275:1593-1599.

Schulz M, Ross B, Pantev C (2003) Evidence for training-induced crossmodal reorganization of cortical functions in trumpet players. Neuroreport 14:157161.

Schwenkreis P, El Tom S, Ragert P, Pleger B, Tegenthoff M, Dinse HR (2007) Assessment of sensorimotor cortical representation asymmetries and motor skills in violin players. Eur J Neurosci 26:3291-3302.

Seitz AR, Kim R, Shams L (2006) Sound facilitates visual learning. Curr Biol 16:1422-1427.

Seitz AR, Kim R, van Wassenhove V, Shams L (2007) Simultaneous and independent acquisition of multisensory and unisensory associations. Perception 36:1445-1453.

Serences JT (2008) Value-based modulations in human visual cortex. Neuron 60:1169-1181.

Shams L, Seitz AR (2008) Benefits of multisensory learning. Trends Cogn Sci 12:411-417.

Shiffrin RM, Schneider W (1977) Controlled and automatic human information processing: II. Perceptual learning, automatic attending, and a general theory. Psychol Rev 84:127-190.

Shiffrin RM, Schneider W (1984) Automatic and controlled processing revisited. Psychol Rev 91:269-276.

Shuler MG, Bear MF (2006) Reward timing in the primary visual cortex. Science 311:1606-1609.

Smolka MN, Buhler M, Klein S, Zimmermann U, Mann K, Heinz A, Braus DF (2006) Severity of nicotine dependence modulates cue-induced brain activity in regions involved in motor preparation and imagery. Psychopharmacology (Berl) 184:577-588.

Stanford TR, Quessy S, Stein BE (2005) Evaluating the operations underlying multisensory integration in the cat superior colliculus. J Neurosci 25:64996508.

Stein BE, Meredith MA (1993) The merging of the senses. Cambridge, Mass.: MIT Press. 
Stewart J, de Wit H, Eikelboom R (1984) Role of unconditioned and conditioned drug effects in the self-administration of opiates and stimulants. Psychol Rev 91:251-268.

Stilla R, Sathian K (2008) Selective visuo-haptic processing of shape and texture. Hum Brain Mapp 29:1123-1138.

Swayne OB, Teo JT, Greenwood RJ, Rothwell JC (2009) The facilitatory effects of intermittent theta burst stimulation on corticospinal excitability are enhanced by nicotine. Clin Neurophysiol 120:1610-1615.

Symes E, Ellis R, Tucker M (2007) Visual object affordances: object orientation. Acta Psychol (Amst) 124:238-255.

Takada M, Hattori T (1987) Organization of ventral tegmental area cells projecting to the occipital cortex and forebrain in the rat. Brain Res 418:27-33.

Tal N, Amedi A (2009) Multisensory visual-tactile object related network in humans: insights gained using a novel crossmodal adaptation approach. Exp Brain Res 198:165-182.

Tanabe HC, Honda M, Sadato N (2005) Functionally segregated neural substrates for arbitrary audiovisual paired-association learning. J Neurosci 25:64096418.

Tiffany ST (1990) A cognitive model of drug urges and drug-use behavior: role of automatic and nonautomatic processes. Psychol Rev 97:147-168.

Tiffany ST, Carter BL (1998) Is craving the source of compulsive drug use? J Psychopharmacol 12:23-30.

Tiffany ST, Conklin CA (2000) A cognitive processing model of alcohol craving and compulsive alcohol use. Addiction 95 Suppl 2:S145-153.

Tobler PN, Fiorillo CD, Schultz W (2005) Adaptive coding of reward value by dopamine neurons. Science 307:1642-1645.

Tucker M, Ellis R (1998) On the relations between seen objects and components of potential actions. J Exp Psychol Hum Percept Perform 24:830-846.

Vainio L, Ellis R, Tucker M (2007) The role of visual attention in action priming. Q J Exp Psychol (Colchester) 60:241-261.

Vezina P (2004) Sensitization of midbrain dopamine neuron reactivity and the selfadministration of psychomotor stimulant drugs. Neurosci Biobehav Rev 27:827-839.

Vezina P, Lorrain DS, Arnold GM, Austin JD, Suto N (2002) Sensitization of midbrain dopamine neuron reactivity promotes the pursuit of amphetamine. J Neurosci 22:4654-4662.

Vitay J, Hamker FH (2007) On the Role of Dopamine in Cognitive Vision In: Attention in Cognitive Systems. Theories and Systems from an Interdisciplinary Viewpoint, pp 352-366. Heidelberg: Springer Berlin.

Volkow ND, Li TK (2004) Drug addiction: the neurobiology of behaviour gone awry. Nat Rev Neurosci 5:963-970.

Volkow ND, Fowler JS, Wang GJ (2002) Role of dopamine in drug reinforcement and addiction in humans: results from imaging studies. Behav Pharmacol 13:355-366.

Volkow ND, Fowler JS, Wang GJ (2004) The addicted human brain viewed in the light of imaging studies: brain circuits and treatment strategies. Neuropharmacology 47 Suppl 1:3-13. 
Volkow ND, Fowler JS, Wang GJ, Baler R, Telang F (2009) Imaging dopamine's role in drug abuse and addiction. Neuropharmacology 56 Suppl 1:3-8.

Volkow ND, Wang GJ, Fowler JS, Logan J, Gatley SJ, Wong C, Hitzemann R, Pappas NR (1999) Reinforcing effects of psychostimulants in humans are associated with increases in brain dopamine and occupancy of $D(2)$ receptors. J Pharmacol Exp Ther 291:409-415.

Volkow ND, Wang GJ, Telang F, Fowler JS, Logan J, Childress AR, Jayne M, Ma Y, Wong C (2006) Cocaine cues and dopamine in dorsal striatum: mechanism of craving in cocaine addiction. J Neurosci 26:6583-6588.

Wallace MT, Stein BE (2007) Early experience determines how the senses will interact. J Neurophysiol 97:921-926.

Wallace MT, Wilkinson LK, Stein BE (1996) Representation and integration of multiple sensory inputs in primate superior colliculus. J Neurophysiol 76:1246-1266.

Waters AJ, Shiffman S, Bradley BP, Mogg K (2003) Attentional shifts to smoking cues in smokers. Addiction 98:1409-1417.

Weisberg J, van Turennout M, Martin A (2007) A neural system for learning about object function. Cereb Cortex 17:513-521.

WHO (1996) ICD-10: International Statistical Classification of Diseases and Related Health Problems : Alphabetical Index (Hardcover).

WHO (2002) The world health report 2002: Geneva, World Health Organization.

WHO (2008) WHO Report on the Global Tobacco Epidemic, 2008: The MPOWER package: Geneva, World Health Organization.

Willuhn I, Steiner H (2006) Motor-skill learning-associated gene regulation in the striatum: effects of cocaine. Neuropsychopharmacology 31:2669-2682.

Willuhn I, Steiner H (2009) Skill-memory consolidation in the striatum: critical for late but not early long-term memory and stabilized by cocaine. Behav Brain Res 199:103-107.

Willuhn I, Sun W, Steiner H (2003) Topography of cocaine-induced gene regulation in the rat striatum: relationship to cortical inputs and role of behavioural context. Eur J Neurosci 17:1053-1066.

Wilson SJ, Sayette MA, Fiez JA (2004) Prefrontal responses to drug cues: a neurocognitive analysis. Nat Neurosci 7:211-214.

Winkielman P, Berridge KC, Wilbarger JL (2005) Unconscious affective reactions to masked happy versus angry faces influence consumption behavior and judgments of value. Pers Soc Psychol Bull 31:121-135.

Wise RA (1980) The dopamine synapse and the notion of pleasure centers in the brain. 3:91-95.

Wise RA (2004) Dopamine, learning and motivation. Nat Rev Neurosci 5:483-494.

Wittenberg GF, Werhahn KJ, Wassermann EM, Herscovitch P, Cohen LG (2004) Functional connectivity between somatosensory and visual cortex in early blind humans. Eur J Neurosci 20:1923-1927.

Yalachkov Y, Kaiser J, Naumer MJ (2009) Brain regions related to tool use and action knowledge reflect nicotine dependence. J Neurosci 29:4922-4929.

Yalachkov Y, Kaiser J, Naumer MJ (2010) Sensory and motor aspects of addiction. Behav Brain Res 207:215-222. 
Yan X, Jiang Y, Wang J, Deng Y, He S, Weng X (2009) Preconscious attentional bias in cigarette smokers: a probe into awareness modulation on attentional bias. Addict Biol 14:478-488.

Yang Z, Xie J, Shao YC, Xie CM, Fu LP, Li DJ, Fan M, Ma L, Li SJ (2009) Dynamic neural responses to cue-reactivity paradigms in heroin-dependent users: an fMRI study. Hum Brain Mapp 30:766-775. 

Acknowledgements 


\section{Acknowledgements}

After two and a half years working on the studies reported here I am more than convinced that a scientific career is not suitable for those people who do not truly enjoy and believe in what they are doing and are not ready to spend some endless nights in the lab. However, even if these requirements are met, one can only hardly succeed in pursuing his goals without the support of his family, friends and colleagues. Happily, I dare to say that I enjoyed their help during the whole time and wish therefore to express my deepest gratitude to...

... Marcus J. Naumer for introducing me to the field of functional neuroimaging and providing me with a flawless support in every way he could.

... Jochen Kaiser for creating such a wonderful and unique place like the Institute of Medical Psychology (IMP). I can not dream of a better place to work.

... both of them for appreciating my research ideas, encouraging me to pursue them and being not only great mentors but also good friends.

... Rainer Goebel for being my supervisor and giving me the opportunity to accomplish my Ph.D. thesis at Maastricht University.

... the members of the assessment committee Prof. Dr. Bernadette Jansma, Prof. Dr. Leo Blomert, Dr. Amanda Kaas, Prof. Dr. Andrea Kübler and Dr. Matt Field for reviewing my thesis.

... Jasper van den Bosch for the Dutch translation of the summary.

... Christian Altmann, Cornelius Abel, Sandra Anti, Anastasia Betaki-Kallinikidou, Christoph Bledowski, Jason Chan, Marie Deußer, Derya Dogruel, Oliver Döhrmann, Monika Geis-Dogruel, Linda Heinemann, Saskia Helbling, Sascha Otterbein, Benny Rahm, Maria Rieder, Maren Schmidt-Kassow, Jasper van den Bosch, Sarah Weigelt and all other people from the IMP, Max Planck-Institute for Brain Research and the Brain Imaging Center in Frankfurt for being wonderful and supportive colleagues and friends.

... Dr. Siegbert Reiß, Prof. Dr. Karin Schermelleh-Engel, Prof. Dr. Klaudius Siegfried and Prof. Dr. Ruxandra Sireteanu for inspiring me and awakening my interest for the research fields of experimental psychology, neuroscience and clinical psychology; Prof. Dr. Dr. Kießling for encouraging me to pursue my goals in spite of all the difficulties.

... Daniel, Gisi, Sebastian, Theresa, Christian, Jörg, Matthias, Philipp, Christopher und Stefan, deren Freundschaft mir eine Ehre ist.

... Благодаря от цялото си сърце на родителите ми Лиляна и Васил Ялъчкови, както и на сестра ми Ваня и дядо ми Стоян, чиято обич и подкрепа значат изключително много за мен. Благодаря ви, че вярвате в мен!

... Благодаря на приятелите ми, които са винаги до мен, дори когато ни делят хиляди километри. Благодаря ви, че с всяка изминала година приятелството ни става по-силно! 
Curriculum Vitae 


\section{Curriculum Vitae}

Yavor Vasilev Yalachkov was born on October $8^{\text {th }} 1985$ in Plovdiv, Bulgaria. After completing his high school education at the English Language School "Plovdiv" in June 2004, he moved to Frankfurt am Main, Germany. In October of the same year he started his psychology studies at the Goethe-University. In the following four years he focused on biopsychology and clinical psychology and developed a particular interest for functional neuroimaging and addiction after completing several internships in these areas. In July 2008 he obtained his M.Sc. in psychology after accomplishing his thesis on "Sensorimotor brain networks as neural correlates of nicotine dependence". During the same year he started working on his Ph.D. thesis at the Institute of Medical Psychology at Goethe-University. In parallel to this, in October 2008 he took up his studies in medicine at Goethe-University. Yavor Yalachkov has received a fellowship from the German National Academic Foundation and has been awarded the "Sabine Grüsser-Sinopoli Award" for young scientists by the German Medical Psychology Society. He is also a lecturer for "Functional Neuroimaging of Addiction" in the curriculum "Addiction Primary Health Care" organized by the Academy of Physicians' Training in Rheinland-Pfalz. Currently Yavor Yalachkov is a postdoctoral researcher at the Institute of Medical Psychology and in the third year of his medical studies at the Goethe-University in Frankfurt am Main.

\section{Publications in peer-reviewed journals}

Yalachkov Y, Kaiser J, Naumer MJ. (2010) Sensory and motor aspects of addiction. Behavioural Brain Research, 207(2):215-22.

Naumer MJ, Ratz L, Yalachkov Y, Polony A, Doehrmann O, Müller NG, Kaiser J, Hein G. (2010) Visuo-haptic convergence in a cortico-cerebellar network. European Journal of Neuroscience 31(10):1730-1736.

Yalachkov Y, Kaiser J, Naumer MJ. (2009) Brain regions related to tool use and action knowledge reflect nicotine dependence. Journal of Neuroscience, 29, 49224929.

\section{Submitted manuscripts}

Yalachkov Y, Naumer MJ. Involvement of action-related brain regions in nicotine addiction. Submitted to Journal of Neuroscience.

Yalachkov Y, Kaiser J, Görres A, Seehaus A, Naumer MJ. Smoking experience modulates the cortical integration of vision and haptics. Submitted to Cerebral Cortex. 


\section{Manuscripts in preparation for submission}

Yalachkov Y, Kaiser J, Görres A, Seehaus A, Naumer MJ. Sensory modality of smoking cues modulates neural cue-reactivity. In preparation for submission to Archives of General Psychiatry.

Yalachkov Y, Kaiser J, Roeper J, Naumer MJ. Neurobiologische und neurokognitive Grundlagen der Sucht. In preparation for submission to Zeitschrift fuer Psychiatrie, Psychologie und Psychotherapie.

Yalachkov Y, Kaiser J, Naumer MJ. Multisensory investigations of addiction. In preparation for submission to Archives of General Psychiatry.

Naumer MJ*, Yalachkov Y*, Polony A, Doehrmann O, Kaiser J. Complementary contributions of the cerebellum and temporo-parietal cortex to object-related visuohaptic integration. In preparation for submission to Current Biology.

(* = equal contribution)

\section{Conference presentations}

- 11th Annual Meeting of the International Multisensory Research Forum (Liverpool)

- Psychologie und Gehirn (Greifswald)

- World Congress of Biological Psychiatry (Paris)

- 35. Arbeitstagung Psychophysiologie und Methodik (Leipzig)

- Gemeinsamer Kongress der Deutschen Gesellschaft für Medizinische Psychologie und der Deutschen Gesellschaft für Medizinische Soziologie (Jena)

- Psychologie und Gehirn (Magdeburg)

- Xth International Conference on Cognitive Neuroscience (Bodrum)

- 9th Annual Meeting of the International Multisensory Research Forum (Hamburg)

- 8th Annual Meeting of the International Multisensory Research Forum (Sydney)

\section{Contact information}

Yavor Yalachkov

Institut für Medizinische Psychologie

Klinikum der Goethe-Universität

Heinrich-Hoffmann-Str.10

D-60528 Frankfurt am Main

Tel.: +49(0) 69-6301-5676

Fax: +49(0) 69-6301-7606

Yalachkov@med.uni-frankfurt.de

www.imp.uni-frankfurt.de 